\title{
Evidence for Tidal Interaction and Merger as the Origin of Galaxy Morphology Evolution in Compact Groups
}

\author{
R. Coziol \\ Departamento de Astronomía, Universidad de Guanajuato \\ Apartado Postal 144, 36000 Guanajuato, Gto, México \\ rcoziol@astro. ugto.mx \\ I. Plauchu-Frayn \\ Departamento de Astronomía, Universidad de Guanajuato \\ Apartado Postal 144, 36000 Guanajuato, Gto, México \\ plauchuf@astro. ugto.mx
}

\begin{abstract}
We present the results of a morphological study based on NIR images of 25 galaxies, with different levels of nuclear activity (star formation or AGN), in 8 Compact Groups of Galaxies (CGs). We perform independently two different analysis: a study of the deviations of isophotal levels from pure ellipses and a study of morphological asymmetries. The results yielded by the two analysis are highly consistent. For the first time, it is possible to show that deviations from pure ellipses are produced by inhomogeneous stellar mass distributions related to galaxy interactions and mergers.

We find evidence of mass asymmetries in $74 \%$ of the galaxies in our sample. In $59 \%$ of these cases, the asymmetries come in pairs, and are consistent with tidal effects produced by the proximity of companion galaxies. The symmetric galaxies are generally small in size or mass, inactive, and have an early-type morphology. They may have already lost their gas and least attached envelop of stars to their more massive companions.

In $20 \%$ of the galaxies we find evidence for cannibalism: a big galaxy swallowing a smaller companion. In $36 \%$ of the early-type galaxies the color gradient is positive (blue nucleus) or flat. Summing up these results, as much as $52 \%$ of the galaxies in our sample could show evidence of an on going or past mergers. Our observations also suggest that galaxies in CGs merge more frequently under "dry" conditions (that is, once they have lost most of their gas).
\end{abstract}


The high frequency of interacting and merging galaxies observed in our study is consistent with the bias of our sample towards CGs of type B, which represents the most active phase in the evolution of the groups. In these groups we also

find a strong correlation between asymmetries and nuclear activity in early-type galaxies. This correlation allows us to identify tidal interactions and mergers as the cause of galaxy morphology transformation in CGs.

Subject headings: galaxies: interactions - galaxies: evolution

\section{Introduction}

It is largely accepted that galaxy formation and evolution are influenced, if not mainly driven, by interactions with their environment. Interactions, in particular, are assumed to be responsible for the phenomenon of segregation of morphologies with radius observed in cluster of galaxies (Oemler 1974; Dressler 1980). The exact nature of these interactions, however, is still largely unknown. The problem is complicated by the large range of properties presented by the environment of galaxies, but also because these properties may equally evolve with time (for example, during the progressive formation of large scale structures, where most galaxies are embedded).

In cluster of galaxies, one possible mechanism for morphology transformation is ram pressure stripping (Gunn \& Gott 1972). According to this model, a late-type spiral galaxy, falling at high velocity into a system having a high density of intergalactic gas, feels a pressure that strips the gas off from its disk. The remaining galaxy, which is now deficient in gas, takes an earlier-type appearance. However, for such mechanism to work, clusters of galaxies must already be rich in intergalactic gas, the origin of which is still a debated matter (e.g. Valageas et al. 2003). It may also be difficult to understand how a late-type spiral galaxy with a small bulge can transform into a larger bulge early-type galaxy by simply losing the gas from its disk (Dressler 1980).

Avoiding these problems, it was proposed that gas rich spirals falling at high velocity into a rich galaxy environment would experience "galaxy harassment" (Moore et al. 1996, 1998). According to this model, the disk of a late-type spiral suffers many deformations from harassment, leading to some gas being rip off from the disk, but mostly flowing toward the center of the galaxy, where it could trigger multiple bursts of star formation, which will contribute in increasing its bulge (and possibly also fueling and AGN).

Although galaxy harassment could easily explain the formation of dwarf elliptical and spheroidal galaxies in clusters (Moore et al. 1996), the formation of luminous elliptical and 
S0 galaxies may require more dramatic events. One such event, which is credited to create mostly elliptical galaxies, is merger (Toomre 1977; Merritt 1984). Alternatively, massive inflows of gas, produced by tidal forces originating from galaxy-galaxy interaction, or galaxycluster interaction (that is, the galaxy interacting with the mass of the whole cluster), may trigger intense starburst episodes in the center of a galaxy (and also fuel an AGN), depleting its disk of gas and increasing the mass of its bulge (Byrd \& Valtonen 1990; Mihos \& Hernquist 1996; Hibbard \& Mihos 1995; Henriksen \& Byrd 1998; Fujita 1998).

It is usually understood that merger and tidal interaction are mechanisms that work more efficiently when the density of galaxies is high and the velocity dispersion of the aggregate of galaxies is low. These conditions are different than those favoring ram pressure stripping and galaxy harassment. In fact, these conditions fit best those encountered in Compact Groups of Galaxies (CGs; for a definition of CGs see Hickson 1982 or Hickson et al. 1992). We expect, consequently, to see more clearly the effects of such processes in these systems.

Evidence for interaction of galaxies in CGs were found in morphological studies (Mendes de Oliveira \& Hickson 1994) and in analysis based on the distribution of neutral and molecular gas (Leon et al. 1991; Verdes-Montenegro et al. 2001). The effects expected from tidal interactions were also shown to be consistent with the nature and level of activities (starburst and AGN) encountered in CGs (Coziol et al. 1998a, 1998b, 2000, 2004). What may still be missing, however, is a clear view of the final phase of the evolutionary process of galaxies in CGs, and evidence that tidal interactions and mergers produce the morphological transformations predicted by the models.

According to the Hierarchical Galaxy Formation theory, the end product of galaxy evolution through tidal interactions and mergers should be massive early-type galaxies. Consistent with this prediction, Hickson et al. (1988) have found the number of early-type galaxies in CGs to be significantly higher than in the field. More recently, Andernach \& Coziol (2006) have shown that CGs isolated from large scale structures possess a clear excess of S0 galaxies compared to the field, which implies that their formation must certainly be favored in these systems. Yet, evidence of "recent" mergers does not seem as obvious as expected. One such evidence, in particular, should be elliptical galaxies with blue nuclei. According to King's merger model (1977) the blue nucleus of an elliptical galaxy is a product of massive stars migrating towards the center of the galaxy by dynamical relaxation. Alternatively, blue nuclei could also be the signs of recent bursts of star formation. Indeed, there are many examples in the literature (both observational and theoretical) connecting mergers to intense starburst episodes. In CGs, therefore, such evidence should be predominant. Surprisingly, Zepf et al. (1991) have determined the fraction of "blue" elliptical galaxies in CGs to be only 
a few percent. Taken at face value, this result suggests either mergers are not as frequent as expected in these systems or past mergers (or starbursts) are too old to be observed in the optical. However, it seems difficult to reconcile the hypothesis of old ages for the mergers, and consequently for the formation of the CGs, with the numerous examples of on-going interactions encountered in CGs, as described above.

As Coziol et al. (2004) have shown, it seems obvious now that the dynamical behavior of CGs does not follow the predictions made by the "fast merger" model (Gómez-Flechoso \& Domínguez-Tenreiro 2001). For example, Hickson et al. (1988) have found the number of early-type galaxies increasing with the velocity dispersion of the group, instead of decreasing as predicted by the model. Coziol et al. (2004) have found the same behavior correlated to a low level of activity, suggesting that CGs with higher velocity dispersions are more evolved. One possible way out of this dynamical dilemma is to assume different formation times for CGS (Coziol et al. (2004): if massive CGs (those with high velocity dispersions) formed before those having lower masses (low velocity dispersion systems), we would necessarily expect them to be more evolved today.

In their spectral analysis of 91 galaxies in 27 CGs, taken from the sample of Hickson (Hickson Compact Groups or HCGs: Hickson 1982), Coziol et al. (2004) have defined a global activity index for the groups, which they used, in combination with the average morphological type of the galaxies in each group, to establish three evolutionary types. The level of evolution of a group was proposed to increase from type A (low velocity dispersion CGs, where galaxies are active and late-type), to type B (intermediate velocity dispersion CGs, where interactions and possibly mergers are on going), to type $\mathrm{C}$ (high velocity dispersion CGs, where galaxies are inactive and early-type). This classification offers a simple explanation for the apparently low frequency of merger remnants encountered in CGs: because mergers happen on average at earlier time during the formation of the groups, observational traces of these events must be difficult to distinguish in the optical. On the other hand, it may still be possible to observe such traces in the NIR, under the form of blue nucleus or stellar mass asymmetries.

In the present article, we present the results of a morphological study of galaxies in CGs, based on NIR images ( $\mathrm{J}$ and $\mathrm{K}^{\prime}$ ). Our goals are to test the hypothesis of different formation time for CGs and to determine the role played by tidal interactions and mergers on the evolution of their member galaxies. For our analysis, we apply independently two different methods: the fitting of elliptical ellipses on the isophotal levels of the galaxies and a determination of their levels of asymmetry. The plan for the article is the following. In Section 2, we present the characteristics of our sample of CGs. In Section 3, we describe our observations and the reduction process. In Section 4, we explain the two methods used 
for our analysis. The results are presented in Section 5, followed, in Section 6, by a short discussion. Our conclusions are exposed in Section 7.

\section{Selection and properties of the sample}

The sample for our NIR analysis consists of 8 HCGs (25 galaxies), taken from the sample previously studied in spectroscopy by Coziol et al. (2004). The physical properties of the groups are presented in Table 1. The properties of the galaxies are given in Table 2. Note that our sample is biased towards CGs of evolutionary type B. This bias was not intentional, but reflects the fact that the NIR observations were carried out before the results of the spectra analysis were available.

The advantage of observing in the NIR is that light in this band is assumed to trace the distribution of the oldest stellar population of a galaxy and, consequently, to follow the luminous mass. In order to verify this assumption we have compiled in Table 3 the NIR magnitudes in J, H and $\mathrm{K}$ from 2MASS for most of the galaxies in our sample. In Figure 1, we compare the colors of our galaxies with the average color sequences traced by different stellar spectroscopic classes, as given by Frogel et al. (1978).

The colors of HCG 56a are typical of Supergiant stars reddened by extinction (Frogel et al. 1987), which is consistent with its spectral classification as a starburst galaxy. The colors of HCG 56b are typical of a power-law (Kotilainen \& Ward 1994), which is consistent with its spectral classification as an AGN. The colors of the rest of our galaxies in our sample are those typical of elliptical and S0 galaxies (Sadler 1984; Bender et al. 1993). The slightly blue $H-K$ colors observed in four of our galaxies can be explained by close interactions (HCG 79b and HCG 98a), and traces of younger stellar populations (HCG 88c and HCG 94a), as our analysis will show. In general, we conclude that the NIR light in our galaxies trace the distribution of the luminous mass.

\section{Observation and reduction}

The observations were carried out with the 2.12-m telescope of the Observatorio Astronómico Nacional, located in the Sierra San Pedro Mártir in Baja California, México, during two different runs: the first one from the 3 to the 6 of September 2001, the second one from the 4 to the 6 of April 2002. The images were obtained using the IR Camera CAMILA (Cruz-González et al. 1994), which is based on four NICMOS3 detectors with $256 \times 256$ pixels, sensitive from 1 to $2.5 \mu \mathrm{m}$. This instrument includes a diaphragm and a 
wheel with 12 filters, which are cooled to reduce the background radiation level. The optical system, which consists of a mirror and a focal reducer designed for the f/4.5 secondary, gives a $3.6 \times 3.6$ sq. arcminutes field of view, corresponding to a plate scale of 0.85 arcsecond per pixel. During our observations, the nights were clear, with an average seeing at the telescope of 1.1 and 0.9 arcseconds in $\mathrm{J}$ and $\mathrm{K}^{\prime}$, respectively (FWHM of a standard star used for focus).

For each galaxy, $\mathrm{J}(\lambda=1.275 \mu \mathrm{m})$ and $\mathrm{K}^{\prime}(\lambda=2.125 \mu \mathrm{m})$ images were obtained following a standard procedure. To avoid saturation of the detector, 5 "coadd" images in $\mathrm{J}$ (respectively 10 in $\mathrm{K}^{\prime}$ ) of 20 seconds (respectively 1 seconds in $\mathrm{K}^{\prime}$ ) were added together to form one image of 100 seconds (respectively 10 seconds in $K^{\prime}$ ). The total integration time for each galaxy, as indicated in Table 2, was reached by adding a sequence of images taken in a mosaic way. Because of the relatively large size of the detector compared to the dimension of the galaxies, this mosaic technique allows to determine the sky background while continuously integrating on the object.

For photometry calibration, 3 standard stars were observed: FS14, FS18 and FS21. These stars were taken from the UKIRT fundamental standard stars list, available on the WEB page of the observatory 1 . For reduction purposes, dome flats were also obtained with a 60 -watt bulb, connected to a potentiometer that was used at low intensity.

The reduction and photometry calibration were done using standard routines in IRAF. First, the images were trimmed, using IMCOPY, to reduce the vignetting effect (CAMILLA at the time was showing a strong vignetting effect on one of its quadrant). Using IMSURFIT (with the option RESPONSE) a surface was then fitted on the combined dome flats (using a spline3 function with order one on both dimensions), obtaining a normalized flat field image. This normalized flat field image was divided from each target image using IMARITH. To eliminate the sky contribution, median sky images, obtained from the combination of four adjacent images in the mosaic series, were subtracted from each corresponding image in the sequence. The $(x, y)$ coordinates of the target object in each image were then measured using the circular aperture photometry option (a) in IMEXAMINE. These positions were used to determine the maximum size of the final combined image. All the images were then trimmed (with IMCOPY) to produce frames with the same dimension and where the object occupies approximately the same position. Before combining the frames, the position of the object in each image was measured once again (the same way as before) to estimate the shifts (a fraction of pixel) needed to put it exactly at the same position (using IMSHIFT). From the frames, a mean image was finally obtained using IMCOMBINE. Note that, once the standard "recipe" is established, the use of IRAF scripts allows reducing a whole series

\footnotetext{
1 http://www.astrossp.unam.mx/estandar/
} 
of images at the same time, which accelerates significantly the reduction process.

The instrument magnitudes for the standard stars were obtained using the PHOT package in IRAF. Calibration equations were calculated by fitting linear regressions on the observation, using the average atmospheric extinction coefficients for San Pedro Mártir, as previously determined by Tapia et al. (1986). Due to the low number of standard stars observed, the uncertainties on the calibrated magnitudes are relatively high: \pm 0.04 in J and \pm 0.07 in $\mathrm{K}^{\prime}$.

\section{Description of the analysis methods}

We applied independently two different methods for our analysis. The first one consisted in fitting elliptical isophotes over the image of the galaxies. A study of the deviations from pure ellipses yields information on the morphology and mass distribution of the different stellar populations (depending on the filter used). A detailed description of the method and how it is used to search for evidence of past interactions and mergers in galaxies can be found in Barth et al. (1995). Note that because we are observing in the NIR, the results yielded by our analysis are easier to interpret in terms of inhomogeneous stellar mass distributions. This is because our NIR images are not affected by stellar formation, interstellar gas or dust extinction (as we demonstrated in Section 2).

The second method consisted in determining the levels of asymmetry of the galaxies (Abraham et al. 1994, 1996; Conselice 1997; Conselice \& Bershady 2000). The application of the technique is straightforward. First the center of the galaxy is determined. This correspond to the point $(x, y)$ in our images ( $x$ and $y$ being real) where the intensity is maximum. The center is determined within a circle of about two pixels, which is slightly bigger than the average size of the PSF. Then the image is rotated by $180^{\circ}$ around this center. After verifying that the location of the maximum has not shifted (otherwise we correct for this shift), we subtract the rotated image from the original one. In the residual image the asymmetries appear under the form of excesses of light (together with their negative images at $180^{\circ}$; see Figures 30 to 35 ). The level of asymmetry within a circular aperture of radius $R$ is calculated using the following formula (Conselice 1997):

$$
A^{2} \equiv \frac{\sum^{R} 1 / 4\left(I_{0}-I_{180}\right)^{2}}{\sum^{R} I_{0}^{2}}
$$

Where $I_{0}$ is the intensity in the original image and $I_{180}$ the intensity in the rotated image. The term $1 / 4=(1 / 2)^{2}$ is the correct normalization factor. It yields a level of asymmetry 
between 0 (completely symmetric) and 1 (completely asymmetric).

Because the galaxies in our sample are not isolated, the value of the maximum asymmetry $\left(A_{\max }\right)$ in our analysis is arbitrary, and not representative of the level of symmetry of the galaxy itself. The explanation is the following. As we integrate further out along the radius to calculate $A$, the presence of companion galaxies becomes more and more important. Therefore, we stop our analysis using the largest radius possible that minimizes this contribution. Because the asymmetry index is cumulative, its maximum is almost always attained at the maximum radius of integration. But since this radius is arbitrary, the maximum asymmetry is consequently also arbitrary.

What indicates symmetry in our analysis is the smoothness of the $A / A_{\text {max }}$ curve with radius (see Figures 30 to 35 ). Comparing with the residual images $\left(I_{0}-I_{180}\right)$, it is easy to verify that in our analysis asymmetries appear as structures in the $A / A_{\max }$ curve. The amplitudes of these structures are proportional to their relative intensities. Note that because of the cumulative nature of the index, asymmetries near the maximum radius may become more difficult to detect in the $A / A_{\max }$ curve. Especially if they have low intensities or if other asymmetries are located directly opposite to their positions. However, the presence of these asymmetries can always be detected in the residual images.

Our asymmetry analysis applies to the inner part of the galaxies. It is not sensible to intergroup light or sky gradients. Our method also minimizes the possible contamination by foreground stars. Usually, we were able to eliminate most of these stars in advance. This was done by masking the stars (using Imedit in IRAF). Only in two cases (HCG 88b and HCG 88c) this correction was not possible. This happened because of the position of the contaminating "object" very near the center of the galaxy. Note that in one case, HCG 88b, we are not convinced this object is really a star.

\section{Results}

In Figures 2 to 9, we present the $\mathrm{J}$ images, in negative, of the eight CGs in our study. In each image we also identify the type of nuclear activity shown by the galaxies. The order of presentation follows the sequence of increasing evolution of the groups.

The first group, HCG 88 (Figure 2), is classified as type A and is one of the most active in our sample. Only two (out of four) galaxies were observed: HCG 88b a Seyfert 2 (Sy2) and HCG 88c a star forming galaxy (SFG). This group is also one of the most spatially extended in our sample (covering 8 arcminutes on the sky). This is why the two galaxies are shown separately. 
The presence of spiral arms, visible in both galaxies is consistent with their late-type classification. These two galaxies show a luminous protuberance very near their centers. These were interpreted as stars in SDSS (based only on their colors; Young et al. 2000). According to our study, however, we cannot discard the possibility of a merging galaxy for HCG 88b (see our analysis below).

The next five groups (HCG 37, 40, 56, 79 and 98) were all classified as type B. In this type of CGs we can see many evidences of interactions: extended, mostly deformed, envelopes around the early-type dominant galaxies (HCG 37a, 40a, 56c and 79a), tidal tails (HCG 40e, 56b and 79b) and possibly even common envelops (HCG 56c with 56d, the whole group HCG 79 and HCG 98). These structures are impressive, considering that what we see in our images are mostly stars. The nuclear activity in these systems is a mixture of AGN (Sy2 and LINER) and star formation, this last activity being more frequent in galaxies having later-type morphologies.

From the point of view of activity, the evidence for interactions are missing in the two groups in our sample classified as type C (HCG 74 and HCG 94). This probably has to do with the early-type morphology of the galaxies and to the higher level of evolution assumed for these groups. However, the presence of extended and possibly common stellar envelops, but not homogeneous or symmetric, suggests these systems are not in dynamical equilibrium (Pildis et al. 1995; Nishiura 2000; Da Rocha \& Mendes de Oliveira 2005).

Because the results for the fitted ellipses and asymmetry analysis in the J and K' band turned out to be similar (consistent with the absence of dust extinction), we present only those obtained with the J filter, which have higher signal to noise levels. Results for the ellipse fitting analysis are shown in Figures 10 to 22 . The radii that include $50 \%\left(r_{50}\right)$ and $90 \%\left(r_{90}\right)$ of the light are indicated by two arrows in the upper graph. Details of the morphologies of each galaxy, under the form of photometrically uncalibrated contour profiles, overlayed on the negative J images, are presented in Figures 23 to 29. Finally, the results for the asymmetry analysis are presented in Figures 30 to 35 . For this last analysis we show not only the variations of $A / A_{\max }$ as a function of the radius, but also the residual images $\left(I_{0}-I_{180}\right)$, which we found extremely useful to detect asymmetries and determine their possible origins. Note that a typical asymmetry in these images has a signal to noise level $S / N \sim 10$.

In the following we discuss the results of our analysis group by group, referring the reader to the corresponding images. 


\subsection{CGs of Type A}

The surface brightness profiles of HCG 88b (Figure 10) show significative structures. The most important one, visible at $r \sim 6^{\prime \prime}$ (which also produces a red bump in the color diagram), is related to an object very near the center of the galaxy (also visible in Figure 23a). This object is at the origin of the large amplitude variations in ellipticity, position angle and in the parameters $A_{4}$ and $B_{4}$, observed in the isophotal profiles. Farther away than $r_{50}=9.3^{\prime \prime}$, other variations of high amplitudes appear: the $A_{4}$ terms increases up to a value of 4 and changes from disky $\left(A_{4}>0\right)$ to boxy $\left(A_{4}<0\right)$. This galaxy becomes blue toward its center.

The asymmetry analysis of HCG 88b (Figure 30a) is fully consistent with the results of the ellipse fitting analysis. Within the radius $r_{50}$, we observe a bright asymmetry near the center. In the opposite direction, at $r_{50}=12^{\prime \prime}$, we see another excess of light, partially covered by the negative image of the central object. This second asymmetry, together with lower intensity asymmetries produced by the spiral arms farther away, are at the origin of the variations of the parameters observed at the same radii in the isophotal profiles.

As we mention earlier, our first impression was that the bright asymmetry observed in HCG 88b was due to a foreground star. However, the presence of the second asymmetry suggests another possibility. Indeed, we do not expect a foreground star to be connected with any other asymmetry. It may be, therefore, that what we see is evidence for a special kind of merger called "cannibalism": that is, a massive galaxy "absorbing" or "swallowing" a smaller mass companion. The second bright asymmetry would then be a tidal structure produced by the intrusion of an external entity (which could be the nucleus of a smaller mass galaxy) near its nucleus.

Alternatively, the second asymmetry may also be related to the bar (as suggested by the morphological classification) or to the spiral arms. However, the evidence of a bar in

our images are not compelling and one would need a very asymmetrical arm to explain this asymmetry. Without further observations we cannot reject the hypothesis of a merger for this galaxy.

The analysis for HCG 88c is very similar to that of HCG 88b. This galaxy also shows a blue nucleus and large amplitude variations of the parameters of the elliptical fits (Figure 10). The most important of these variations is produced by an object within $r_{50}$ (Figure $23 \mathrm{~b}$ ). The fainter variations, observed farther away, can be produced by the spiral arms. This is consistent with what we see in the residual image (Figure 30b).

Note that contrary to HCG 88b, we do not observe an excess of light in the direction opposite the location of the brightest asymmetry, which could be interpreted as a possible tidal feature. In this case, therefore, the probability for this object to be a foreground star is 
much higher. The only asymmetries observed in this galaxy would thus be those produced by the spiral arms. There is, consequently, no apparent evidence of interaction in our analysis for this galaxy.

\subsection{CGs of Type B}

The first example of a CG of type B in our sample is HCG 37, which is formed by two large galaxies and a smaller one. The dominant galaxy, HCG 37a, is an elliptical (Figure 23c) with very smooth surface brightness profiles (Figure 11). This galaxy is boxy on almost all its extension. Our analysis shows that the isophotes become more elliptical at large radius, with an axis increasingly deviating from the semi major axis (variations of parameter $B_{4}$ ). In the residual image (Figure 30c), these variations are connected to a large arc-like asymmetry, which increases in intensity toward the northwest. This huge excess of light points toward the two companions galaxies (particularly HCG37b), suggesting a possible gravitational influence. Within $r_{50}=7.7^{\prime \prime}$ we also distinguish two faint structures appearing as two peaks in the $A / A_{\max }$ diagram (first panel in Figure 30c).

The second dominant galaxy, HCG 37b, is a spiral seen almost edge on (Figure 23d). This galaxy becomes blue toward its periphery (Figure 11). The isophotal profiles are very smooth, with almost constant parameters and large errors. This is expected for an edge on spiral galaxy, due to the orientation of the galaxy. Consistent with this orientation, the asymmetry residual image shows an excess of light toward the side directly facing us (Figure 30d). However, we also note a slight excess of light pointing in the direction of HCG 37a to the southeast. This could be interpreted as a tidal effect (in response to what we see in HCG 37a) or it could be that the galaxy is also oriented toward its more massive companion. Both interpretations are consistent with tidal interaction effects.

The last galaxy in this group, HCG 37c, is classified as an intermediate ellipsoid galaxy (S0a; Figure 24a). The isophotal profiles are relatively smooth, with almost constant parameters (Figure 12). The galaxy as a marginally (considering the error) blue center. Except for the important contamination of light seen at large radius, and due to HCG 37a, the asymmetry residual image (Figure 31a) reveals no structures, which is fully consistent with the smooth isophotal profiles.

The second group of type B in our sample is HCG 40. This is a very interesting group, with many evidences for interactions (Figure 4). The dominant galaxy, HCG40a, is elliptical

(Figure 24b). This galaxy possesses a marginally blue center (Figure 12). Although the surface brightness profiles are smooth, the parameters of the fitted ellipses show multiple, 
large amplitude variations, suggesting an inhomogeneous stellar mass distribution. This is confirmed by the asymmetry analysis. In Figure 31b, we observe two bright plume-like asymmetries: one to the north, with maximum intensity at $r=7^{\prime \prime}$ and the other slightly farther than $r=20^{\prime \prime}$. These structures produce the depressions in the parameters $A_{4}$ and variations in $B_{4}$ observed at the same radii in the isophotal profiles.

The second galaxy of this group, HCG40b, is an S0 galaxy (Figure 24c). The isophotal profiles (Figure 13) show large amplitude variations, within and up to $r_{50}$. These variations are related to a bright asymmetry observed to the north (Figure 31c), and a weaker extension to the west. These asymmetries also produce the plateau observed in the $A / A_{\text {max }}$ diagram. Note that there is once again a possibility for the brightest asymmetry to be a foreground star (this would be the third case). The extended excess of light, on the other hand, seems consistent with an interaction feature produced by the proximity of HCG 40c.

This galaxy, HCG 40c, is an edge on spiral (Figure 24d). As expected, the isophotal profiles (Figure 13) are smooth with almost constant parameters (and large errors). However, we distinguish in the residual image (Figure 31d) two bright asymmetries. One is located at $r_{50}=7.1^{\prime \prime}$ to the southeast, and the other a little bit farther to the northwest. Only the first one is clearly visible as a bump in the $A / A_{\max }$ diagram (first panel in Figure $31 \mathrm{~d}$ ). The bright asymmetry to the southeast is connected to a bridge of matter between HCG 40c and HCG 40e. At the same distance, but in the opposite direction, we also observe a fainter asymmetry. The numerous asymmetries observed in this galaxy are consistent with tidal structures produced by interactions (and possibly a bridge of stars) with its three companions (HCG 40a, 40b and 40e).

The galaxy HCG 40d is a barred early-type spiral (Figure 25a), which seems slightly separated from the others. In Figure 14, the presence of the bar can be inferred from the "plateau" appearing slightly beyond $r_{50}=3.7^{\prime \prime}$ in the surface brightness profiles. In the same region the ellipticity increases with the radius, the position angle decreases and the parameters $A_{4}$ and $B_{4}$ show large amplitude variations.

In the residual image (Figure $32 \mathrm{a}$ ), an intense asymmetry appears at $r_{50}$. This asymmetry produces the variations of the isophotal parameters observed at the same radius in Figure 14. We can also distinguish two asymmetries beyond $r=11^{\prime \prime}$, located at the extremities of the disk. The excess of light to the east, just below the asymmetry, is a residue from a subtracted star.

The fact that this galaxy is probably seen near edge on complicates the analysis. Once more, the brighter asymmetry could be due to a foreground star (the fourth case). However, the presence of other accompanying asymmetries may also suggest cannibalism or a global 
response to the proximity of the companions.

The interpretation seems clearer for the last galaxy in this group, HCG 40e. This galaxy is directly connected to HCG 40c by a bridge of stars, as visible in Figure 25b. To decrease the importance of this structure, our analysis concentrates on the most inner part $\left(r<7^{\prime \prime}\right)$. Within this limit, the isophotal profiles are smooth (Figure 14), showing very few variations of the fitted ellipse parameters. The galaxy is boxy and shows the tendency to become blue toward its center. The residual image (Figure 32b) reveals no important asymmetries within $\left(r_{50}=5^{\prime \prime}\right)$, which is fully consistent with the isophotal analysis. Beyond this radius, however, we find two bright asymmetries, one located in the opposite direction to the bridge of stars (northeast), the other more intense toward the west. This last one could be an extension of the bridge. The interpretation of these asymmetries as tidal structures is obvious.

The next group, HCG 56, offers other examples of strongly interacting galaxies. The dominant galaxy in this group, HCG 56a, is a late-type spiral, seen edge on (Figure 25c). Our preliminary analysis of its color (Section 2) suggests that our NIR images may be affected by star formation and significant amount of dust extinction. The effect of extinction could very well explain the peculiar color profile (Figure 15), which suddenly turns red toward the extremity of the disk.

The residual image (Figure 32c) shows the usual pattern for an edge on spiral galaxy, with the side facing us being brighter. This brighter face has a patchy appearance, though, which could be the effect of dust extinction. We distinguish at least two peaks in the $A / A_{\max }$ diagram. One is located within $4^{\prime \prime}<r<9^{\prime \prime}$ and the other start at $r \sim 14^{\prime \prime}$. These asymmetries produce the variations of the parameters $A_{4}$ and $B_{4}$ at the same radii. Once again, the side pointing toward the direction of the other group members (to the north), seems more intense. The possible interpretations are similar to those suggested for HCG 37a.

The second galaxy in this group, HCG 56b, is a barred, lenticular galaxy (Figure 25d). Our preliminary analysis suggests our NIR images may be affected by an AGN, also with significant dust extinction (Section 2). This is consistent with the sudden variations observed near $r_{50}=2.4^{\prime \prime}$ in the isophotal profiles. Like for HCG40d, the bar produces a plateau in the surface brightness profiles around $r_{90}$ (Figure 15). Out of the central region, the isophotal profiles varied smoothly, the eccentricity increasing with the radius and the galaxy changing from disky to boxy.

The residual image (Figure $32 \mathrm{~d}$ ) reveals a bright asymmetry at $r=10^{\prime \prime}$, opposite to the direction of the bridge of stars with HCG 56c. This asymmetry produces the large amplitude variations of the isophotal parameters observed at the same radius.

The S0 galaxy HCG 56c is connected by a bridge of stars to HCG 56b (Figure 26a). 
The color profile (Figure 16) is constant over its entire extension. This galaxy has circular isophotes within $r_{50}=2^{\prime \prime}$ and elliptical ones farther away. The parameters $A_{4}$ vary little, while the parameters $B_{4}$ increases with the ellipticity. The residual image (Figure 33b) reveals no asymmetries. The sudden variation of the position angle observed in the isophotal profiles probably marks the passage from a bulge to a disk.

The analysis for HCG 56d is very similar to the previous one. This lenticular galaxy (Figure 26b) shows smooth, continuously changing profiles, and very few variations of the parameters $A_{4}$ and $B_{4}$ (Figure 16). The residual image (Figure 33b) reveals no asymmetries.

Because of its extreme position in our image, only the isophotal analysis for HCG 56e was possible. This small S0 galaxy (Figure 26c) shows almost no variations in colors (Figure 17), very few variations in ellipticity or of the parameters $A_{4}$ and $B_{4}$. Like for HCG 56c, and $56 \mathrm{~d}$, we would not expect to see asymmetries in this galaxy.

Our next group, HCG 79, contains three major members. The dominant galaxy, HCG 79a, is an early-type spiral (Figure 26d). The surface brightness in $\mathrm{J}$ (we do not have $\mathrm{K}^{\prime}$ for the galaxies in this group) is very smooth (Figure 17). We see small variations of ellipticity and a shift by $18^{\circ}$ of the position angle. The parameter $A_{4}$ is mostly disky and shows small amplitude variations over its entire extension. These variations are accompanied by similar variations of the parameter $B_{4}$. The residual image (Figure 33c) reveals two bright asymmetries. One is located within $r_{50}=6.3^{\prime \prime}$ to the west, and the other farther away to the east. These asymmetries produce the variations of the parameters $A_{4}$ and $B_{4}$ observed at the same radii.

The second galaxy of this group, HCG 79b, is a lenticular galaxy (Figure 27a). Its surface brightness profile shows a bump half way between $r_{50}=5.3^{\prime \prime}$ and $r_{90}$, which seems to influence the values of the other parameters (Figure 18). The residual image for this galaxy (Figure 33d) reveals a very bright asymmetry to the west. This is consistent with the peak between $5^{\prime \prime}<r<10^{\prime \prime}$ in the $A / A_{\max }$ diagram and explains the variations of the isophotal parameters observed at the same radii. This asymmetry is opposite to the direction of the tidal tail and may form an extension to the bridge of stars connecting HCG 79b to HCG 79c.

The bridge of stars (Figure 27b) perturbs the isophotal profiles of HCG 79c (Figure 18). This is confirmed by the residual image (Figure 34a). In the opposite direction to the main asymmetry produced by the bridge we may distinguish a fainter one, within $r_{50}=6.1^{\prime \prime}$. However, since this asymmetry does not seem to show up in the graph of $A / A_{\text {max }}$, which is quite smooth, we would rather classify HCG 79c as symmetric.

The last group of type B in our sample is HCG 98. We have only information on the two first members: HCG $98 \mathrm{a}$ and $98 \mathrm{~b}$. The analysis of this group is complicated due to the 
presence of a foreground star (the only one confirmed by spectroscopy) between HCG 98a and 98b (Figure 27c). This star was deleted from our image before performing our analysis.

The dominant S0 galaxy in this group, HCG 98a, is classified as a barred lenticular, although the bar is not obvious in our images (Figure 27c). The two surface brightness profiles (Figure 19), which look convex between $r_{50}$ and $r_{90}$, may trace the bar. However, we find such evidence quite weak and cannot conclude positively on the presence of such structure.

Within $r_{50}=5.8^{\prime \prime}$, we observe important variations of the ellipticity and position angle. Both parameters, $A_{4}$ and $B_{4}$, show multiple variations of low amplitudes. The residual image (Figure 34b) reveals two major asymmetries. One located within $r_{50}$, the other farther away to the west, and associated to a faint extension to the south. These asymmetries clearly produce the variations of the isophotal parameters. These structures (in particular the most central one) may reflect some advanced merger phase, or correspond to tidal features, resulting from the interaction with HCG $98 \mathrm{~b}$.

For the second galaxy, HCG 98b, we observe (Figure 19) a sudden change of ellipticity and position angle at $r_{50}=2.9^{\prime \prime}$ and multiple low amplitude variations of the parameters $A_{4}$ and $B_{4}$. The residual image (Figure $34 \mathrm{c}$ ) reveals an asymmetry located at $r_{50}$ to the east, which produces the variations observed in the isophotal profiles. We also distinguish an excess of light in the direction of HCG 98a. Once again (for the fifth time), the bright object at $r_{50}=1$ could be a foreground star. The excess of light, on the other hand, seems related to a possible bridge of stars, which would connect HCG 98b to HCG 98a (Figure 7 and Figure 27d).

\subsection{CGs of type C}

The first group of type $\mathrm{C}$ in our sample, HCG 74, is formed by three early-type galaxies, embedded in an apparent common envelop of stars (Figure 8). The dominant galaxy, HCG $74 \mathrm{a}$, is an elliptical with a light protuberance to the west (Figure 28a). This object affects all the isophotal profiles (Figure 20).

In the residual image (Figure 34d), the protuberance corresponds to a bright asymmetry located at $r=5^{\prime \prime}$, with some weaker extensions passing through the center of the galaxy. In opposite direction to this bright feature, to the east, we distinguish a much more diffuse and extended asymmetry. The presence of this pair of asymmetries is an argument in favor of cannibalism (and not a contamination by a foreground star, which would be the sixth example). Supporting this interpretation, we note that the excess of light to the east does 
not point toward HCG 74b or HCG 74c.

The two companions, HCG 74b and HCG 74c (Figures 28b and 28c), show similar isophotal profiles, with very few variations (Figures 20 and 21). Their residual images (Figure 35a and 35b) reveal no asymmetries. Apparently, these galaxies are symmetric.

The second group of type $\mathrm{C}$ in our sample, HCG 94, is very similar to the previous one (Figure 9), with the exception that the two dominant galaxies in this group seem now comparable in size and mass (based on comparable luminosities in the optical and NIR). These two galaxies (Figures 28d and 29) have a blue nucleus (Figure 21 and 22). The isophotal profiles show only small amplitude variations of the fitted ellipse parameters. The residual image of HCG 94a (Figure 35c) reveals one asymmetry just slightly farther than $r_{50}=3.6^{\prime \prime}$ and a weak excess of light forming an arc-like structure from east to north. A similar weak excess of light is observed in HCG 94b (Figure 35d). This arc-like structure extends from west to north. In HCG 94a the brighter asymmetry is consistent with cannibalism, while the two arc-like structures could be tidal structures produced by the interaction of the two galaxies.

\section{Discussion}

The results produced by our two methods, applied independently, are highly consistent. This is of major importance for our analysis. From a detailed comparison of the results, for the first time we can verify that the variations of parameters of fitted ellipses in galaxies reflect inhomogeneous stellar mass distributions, produced by tidal interactions and mergers (Di Tulio 1979; Kormendy 1982; Zaritsky \& Lo 1986; Bender \& Möllenhoff 1987; Barth et al. 1995). The two methods being applied independently, the consistency of the results also puts heavy weights in our observations.

In Table 4, we summarize the results obtained from our asymmetry analysis. A large fraction ( $74 \%$ or 17 out of 23 ) of the galaxies in our sample present evidence of asymmetries related to interactions (we do not count HCG $88 \mathrm{c}$, where the asymmetries are probably related to the spiral arms). Of highly importance for the physical interpretation of our result, in 59\% (10 out of 17) of the asymmetric cases the asymmetries come in pairs. This is as expected if they are the results of tidal forces.

In the seven galaxies where the asymmetry is not in a pair, the excess of light either clearly points toward the projected position of the companions (HCG 37a, 37b, 40b, 56a, 98b), or seems to form part of a common envelop (HCG 94a and 94b). In general, therefore, detected asymmetries in CG galaxies are directly connected to interaction effects. This result 
confirms the analysis previously made by Mendes de Oliveira and Hickson (1994).

For the tidal cases, we identify two possible origins for the asymmetries. In five galaxies (HCG40a, 40c, 40e, 56b and 79b) one asymmetry forms a pair with a bridge or a tail. In the other five galaxies (HCG 88b, 40d, 79a, 98a and 74a), the brightest asymmetry is accompanied by a weaker or more extended one, directly opposite to its position. This we interpret as evidence for a special case of merger, that we call cannibalism: a massive galaxy swallowing a smaller mass companion.

Note that although we cannot eliminate the possibility of contamination by foreground stars to explain the most intense asymmetry in the candidate cannibal galaxies, our interpretation has the advantage to yield a simple explanation for the presence of the second accompanying asymmetry, whose origin, otherwise, would stay undefined. This is the case of HCG 74a, for example, where the excess of light directly opposing the brighter asymmetry does not point toward neither of its two companion galaxies, and consequently cannot be a tidal response to their proximity.

Our conviction in favor of the cannibalism interpretation stands on three points. First, the frequency of cases where a star would coincides with the nucleus of a galaxy in our sample looks suspiciously high $(24 \%, 6$ out of 25$)$. This is considering that the line of sight of the CGs in our sample are not passing through particularly dense stellar regions. Second, we do not expect a bright star to be apparently connected with another asymmetry. In HCG 98, where we are sure the bright object is a star, and in other cases where we have subtracted the stars before doing our analysis, we find no relation between the detected asymmetries and the residue left by the star subtraction. Finally, we do find numerous examples of cannibalism in the local group (e. g. Martin et al. 2004; McConnachie \& Irwin; Belokurov et al. 2006; Zucker et al. 2006; Martin et al. 2006), and we expect consequently such phenomenon to be even more frequent in CGs.

Another evidence in favor of past mergers in our sample is the high frequency observed of early-type galaxies showing a flat or positive color gradient (Zepf et al. 1991; Michard 1999; La Barbera et al. 2003; Ko \& IM 2005). We count nine galaxies with such a feature in our sample: HCG 37a, 37c, 40a, 40b, 56e, 94a, 94b, 98a and 98b. These galaxies are mostly inactive (some activity is observed in $\mathrm{HCG} 37 \mathrm{a} 37 \mathrm{c}$ and $56 \mathrm{e}$, but only at a low level) and asymmetric (except for HCG 37c and 56e, for which the information is missing).

If we consider only the elliptical galaxies, 3 out of 6 are found to be blue in their inner part: HCG40a, 94a, 94b. HCG94a and HCG94b were also reported to be blue in the optical by Pildis et al. (1995). HCG40a, which is marginally blue in our image, was not reported to be blue by Zepf et al. (1991). However, these authors do not present the color profile, 
which is obviously flat in our image. Note that we do not find the color gradient of HCG37a presented by Zepf et al. (1991) to be that blue either (positive slope), although we concur on the flat color profile.

Note that we do not use the level of boxyness as evidence for merger in our study for two reasons. First, this criterion usually apply to elliptical galaxies, assumed to be in a final stage of evolution. Our data suggest a much more complicated picture, the nature boxyness or diskyness of the profiles frequently varying with the radius. This behavior is consistent with the high number of asymmetries observed in our galaxies, which suggest the early-type galaxies in our sample are not in a final stage of evolution. Second, recent simulations have shown that even for normal elliptical galaxies the relation between boxyness and mergers is not as straightforward as previously thought (see Naab et al. 2006a; 2006b), depending very much on the details of the mergers.

Summing up the galaxies with evidence for cannibalism to the peculiar early-type galaxies, as much as $52 \%$ of the galaxies in our sample could thus show evidence for past mergers. This is much higher (6\%) than what was found by Mendes de Oliveira and Hickson (1994), in their morphology analysis based on the optical, or Zepf et al. (1991) in their search for optically blue elliptical galaxies. The frequency of merger remnants we have detected is now much more consistent with what was originally expected in CGs.

Taken at face value, our results on past merger in CGs may seem in contradiction with those found by Zepf et al. (1991) or Mendes de Oliveira and Hickson (1994). However, we have to note that our sample is biased towards CGs of type B, which according to the scenario of evolution proposed by Coziol et al. (2004) represent the most active phase in the evolution of the group. One would obviously expect evidence for past mergers to be more prominent in these systems. Our results, therefore, seems to confirm our classification scheme based on activity.

For the six symmetric galaxies in our sample, we cannot eliminate the possibility of asymmetries at a lower intensity level and higher spatial resolution than reached in our analysis. However, it seems obvious that the level of perturbation in these galaxies must be significantly low compared to that in the other galaxies. These galaxies turn out to be smaller in size (and probably in mass, as judged from their NIR luminosity) than their companions. They are also mostly inactive (the exception is HCG 56d), and have an earlytype morphology. Therefore, it could be that, having smaller masses, these galaxies have already lost most of their envelop of gas and least attached stellar disks to their more massive companions. What is left, would be the components that are more tightly bounded by gravitation to the galaxy, explaining the apparent absence of asymmetries. This description would also fit the entities found near the nuclei in the cannibal candidates. 
From the point of view of nuclear activity, we cannot establish a one to one relation with interaction. Although 77\% (10 out of 13) of the galaxies showing some sort of activity in our sample also show asymmetries related to interactions (again, we do not consider HCG 88c), these cases represent only $55 \%$ of the galaxies with asymmetries. Neither the morphological types of the active galaxies can explain the results: only $50 \%$ of the active galaxies in our sample have a late-type morphology.

However, if we consider the level of evolution of the groups, a clear trend appears. In Table 5, we separated the groups according to their evolutionary type. In the cases of type $\mathrm{A}$ and $\mathrm{C}$ our data are too scarce to establish any firm tendency. But for the groups in type $\mathrm{B}$, we can clearly distinguish a connection between interaction and activity: most of the galaxies that show asymmetries related to tidal interactions and mergers are active, while only five have a late type morphology. The fact that a high number of early-type galaxies are active and perturbed in these systems directly connects tidal interactions and mergers to morphological transformations.

Our observations support the morphological transformations of late-type galaxies into earlier types in CGs. It remains, however, to determine what could be the typical time scale for such transformations. Recent merging models of gas poor galaxies suggest tidal asymmetries in the NIR should be observable over a time scale of the order of $0.4 \pm 0.2$ Gyrs (Van Dokkum 2005). Toledo et al. (2006) found comparable merger time scales in their asymmetry analysis of $42 \mathrm{E} / \mathrm{S} 0$ interacting pairs. On the other hand, Coziol et al. (1998b) have shown that the usual indices for post-starburts in CGs suggest ages higher than 2 Gyrs for the last bursts in the early-type galaxies. Therefore, their seems to be a significant difference between the two time scales. Note that Zefp \& Whitmore (1991) already encountered a similar discrepancy comparing the frequency expected of "blue" elliptical galaxies with the dynamical time scale for the evolution of the groups (the difference is of the order of what we reported above). More generally, Schweizer \& Seitzer (1992) found a similar problem in their study on the origin of elliptical galaxies by mergers.

The hypothesis putted forward to explain the symmetric and cannibalized galaxies may offer a new solution. If mergers in CGs take place under dry conditions, that is, happening after most of the gas has already been ejected, consumed into stars or burned by an AGN, then, without the possibility of extra star formation, the merging remnants would not be expected to be particularly blue (Naab et al. 2006a, 2006b; Van Dokkum 2005). This would give the impression that mergers in CGs are rare and post-starbursts older than they really are.

One way to test this hypothesis is to determine the colors of tidal features in dense environment. In absence of numerous massive stars, these features would not be expected 
to be particularly blue. In their analysis of galaxy interactions in clusters, Conselice \& Gallagher (1999) have shown that tidal structures have a range of colors extending from blue $(B-R \approx 0)$ to red $(B-R \approx 2.5)$. Comparing these colors to those of normal S0 and Elliptcal galaxies (Michard 2000), one can see that these structures are rarely blue (average of 1.46). In table 3 , the galaxies in our sample have an average color $(B-R)$ of 1.63 , which agree relatively well with the distribution of the colors of the tidal structures encountered by Conselice \& Gallagher. These authors suggest what they observe is the result of interactions of the galaxies with the cluster potential. This is also what we propose as the cause of dry merger in CGs.

Other observations consistent with the hypothesis of dry merger in CGs are the following: 1) the absence of antenna like merging systems or luminous AGNs (Coziol et al. 1998a); 2) the fact that star formation is generally low (Allam et al. 1999; Coziol et al. 2000), 3) the detection of galaxies which are deficient in gas (Hutchmeier 1997), 4) the effects expected from truncation of star formation on the evolution of stellar populations (Caon et al. 1994; ; Coziol et al. 1998b; de La Rosa et al. 2006). Finally, the characteristics of the galaxies identified as candidates for dry merger in our sample fit relatively well the definition given of these objects by Bell et al. (2006). The highest prediction made by these authors on the frequency of merger events expected in dense environments are also in good agreement with the frequency of dry merging candidate galaxies we encountered in our sample.

Putting everything together, the scenario that fit better our observations in CGs of type $\mathrm{B}$ seem to be the following. As galaxies join to form a group, the effect of tidal interaction with the potential well of the whole system affect first their gas content: part of the gas passes to the nucleus starting star formation and/or refueling an AGN in gas, and part must go to the intergalactic medium. Starting with spiral galaxies, this process would be expected to produce mostly lenticular galaxies. These galaxies would then start interacting with each other, and at one point merge together, to form one giant elliptical galaxy. Under dry merger conditions, the time scale of the process must be shorter than 2 Gyrs. This implies that CGs of type B formed relatively recently.

\section{Conclusions}

The two analysis applied independently are highly consistent. Both methods suggest the galaxies in CGs are clearly not in equilibrium, but in a process of transformation. The asymmetry analysis allows us to identify the mechanisms behind this process: the galaxies experience the effects of tidal interactions and possibly multiple mergers. 
Concerning tidal interactions, it is not clear what distinguish galaxy-galaxy interactions from galaxy-group interactions. The scenario we propose for the evolution of the groups suggests the difference between the two mechanisms lies in the response of the gas: through galaxy-group interaction, the galaxies would first lose their envelop of gas and would then start interacting and merging with each other under dry conditions.

Combining our observations on asymmetries with information on nuclear activity we also have a definite view about the type of transformations that is taking place in CGs: late-type galaxies transform into earlier types (S0 and elliptical). From our analysis, therefore, we clearly identify tidal interaction and merger as the mechanisms responsible for the transformation of morphology of galaxies in CGs.

As for the global evolution of CGs, our sample is unfortunately still incomplete. We need more information on CGs of type $\mathrm{A}$ and $\mathrm{C}$ in order to be able to test the hypothesis of different formation ages for the CGs. Based on our data alone, however, the possibility that all the galaxies in one CG will merge to form one giant elliptical galaxy cannot be rejected.

This is where the hypothesis of a different formation ages for CGs, depending on their mass, becomes interesting. The first CGs to reach complete evolution would have been located in massive and dense environments. Under such conditions, different groups could have merge with each other to form larger structures (Andernach \& Coziol 2006). Consequently, we would not expect to see "isolated" elliptical galaxies as the product of CGs evolution, since these remnants today would be in larger groups or cluster of galaxies (Yoshioka et al. 2004).

Assuming merger of CGs were more frequent in the past, such events could have played an important role in the formation of large scale structures, like cluster of galaxies (Mihos 2004). Consequently, many galaxies found today in clusters may have first evolved in groups (Ellingson 2003; Mihos 2004). If this is the case, our results would support the idea that the segregation of galaxy morphologies observed in clusters today is mostly a product of tidal interactions and mergers.

We thank the CATT of San Pedro Mártir for the observing time given on the $2.12 \mathrm{~m}$ telescope to realize this project and all the personal of the observatory for their support. We also thank Dr. Ascensión del Olmo Orozco and Dr. Heinz Andernach for discussing parts of this article with us. An anonymous referee is also acknowledge for comments that have helped to improve the clarity and quality of our presentation. Finally, we acknowledge the support of CONACyT under grant 47282-F. This research has made use of the NASA/IPAC Extragalactic Database (NED), which is operated for NASA by the Jet Propulsion Laboratory, California Institute of Technology. 


\section{REFERENCES}

Abraham, R. G., Valdes, F., Yee, H. K. C. \& van den Bergh, S. 1994, ApJ, 432, 75

Abraham, R. G., Tanvir, N. R., Santiago, B. X., Ellis, R. S., Glazebrook, K. \& van den Bergh, S. 1996, MNRAS, 279, L47

Allam, S. S., Tucker, D. L., Lin, H. \& Hashimoto, Y. 1999, ApJ, 522, L89

Andernach, H. \& Coziol, R. 2006, Proc. ESO Workshop: Groups of galaxies in the nearby Universe, ESO Astrophysics Symposia, I. Saviane, V. Ivanov, J. Borissova, eds., Springer-Verlag, 2006, in press (astro-ph/0603295)

Barth, C. S., Coziol, R. \& Demers, S. 1995, MNRAS, 276, 1224

Belokurov, V., Zucker, D. B., Evans, N. W., Wilkinson, M. I. and 29 coauthors 2006, ApJ, 647,111

Bender, R., \& Möllenhoff, C. 1987, A\&A, 177, 71

Bender, R., Burstein, D. \& Faber, S. M. 1993, ApJ, 411, 153

Byrd, G. \& Valtonen, M. 1990, ApJ, 350, 89

Caon, N., Capaccioli, M., D’Onofrio, M., \& Longo, G. 1994, A\&A, 286, 39

Conselice, C. J. 1997, PASP, 109, 1251

Conselice, C. J. \& Gallagher, J. S. 1999, AJ, 117, 75

Conselice, C. J. \& Bershady, M. A. 2000, ApJ, 529, 886

Coziol, R., Ribeiro, A. L. B., Capelato, H. V. \& de Carvalho, R. R. 1998a, ApJ, 493, 563

Coziol, R., de Carvalho, R. R., Capelato, H. V. \& Ribeiro, A. L. B. 1998b, ApJ, 506, 545

Coziol, R., Iovino, A. \& de Carvalho, R. R. 2000, AJ, 120, 47

Coziol, R., Brinks, E., \& Bravo-Alfaro, H. 2004, AJ, 128, 68

Cruz-González, I., Carrasco, L., Ruiz, E., Salas, L., Skrutskie, M., Meyer, M., Sotelo, P., Barbosa, F., Gutiérez, L., Iriarte, A., Cobos, F., Bernal, A., Sánchez, B., Valdéz, J., Argüelles, S., Conconi, P. 1994, in Instrumentation in Astronomy VIII, D. L. Crawford \& E. R. Craine, eds. Proc. SPIE 2198, 774 
Da Rocha, C. \& Mendes de Oliveira, C. 2005, MNRAS, 364, 1069

de la Rosa, I. G., de Carvalho, R. R., Vazdekis, A. \& Barbuy, B 2006, RevMexAA (Serie de Conferencias), 26, 113

Di Tullio, G. A. 1979, A\&AS, 37, 591

Dressler, A. 1980 ApJ, 236, 315

Ellingson, E. 2003, in Galaxy Evolution in Groups and Clusters, C. L. Lobo, M. S. Roos, A. Biviano, eds. Ap\&SS, 285, 9.

Frogel, J. A., Persson, S. E., Aaronson, M. \& Matthews, K. 1978, ApJ, 220, 75

Frogel, J. A. \& Elias, J. H. 1987, ApJ, 313, L53

Fujita, Y. 1998, ApJ, 509, 587

Gómez-Flechoso \& M. A., Domínguez-Tenreiro, R. 2001, ApJ, 549, L187

Gunn, J. E. \& Gott, J. R. 1972, ApJ, 176,1

Henriksen, M. \& Byrd, G. 1996, ApJ, 459, 82

Hibbard, J. E. \& Mihos, J. C. 1995 AJ, 110, 140

Hickson, P. 1982, ApJ, 255, 382

Hickson, P., Kindl, E. \& Huchra J. P. 1988, ApJ, 331, 64

Hickson, P., Kindl, E. \& Auman, J. R. 1989, ApJS, 70, 687

Hickson, P., Mendes de Oliveira, C., Huchra, J. P., \& Palumbo, G. G. 1992, ApJ, 399, 353

Hutchmeier, W. K. 1997, A\&A, 325, 473

Jarret, T. H., Chester, T., Cutri, R., Schneider, S. E. \& Huchra, J. P. 2003, AJ, 125, 525

King, I. R. 1977, in The Evolution of Galaxies and Stellar Populations, B. Tinsley \& R. Larson eds., New Haven: Yale Univ. Obs., p. 418

Ko, J. \& Im, M. 2005, Journal of the Korean Astronomical Society, 39, 3

Kotilainen, J. K. \& Ward, M. J. 1994, MNRAS, 266, 953 
Kormendy, J. 1982, in Morphology and Dynamics of Galaxies, Martinet L., Mayor M., eds., Saas Fee, p. 113

La Barbera, F., Busarello, G., Massarotti, M., Merluzzi, P. \& Mercurio A. 2003, A\&A, 409, 21

Leon, S., Combes, F. \& Menon, T. K. 1998, A\&A, 330, 37

Martin, N. F., Ibata, R. A., Bellazzini, M., Irwin, M. J., Lewis, G. F. \& Dehnen, W. 2004, MNRAS, 348, 12

Martin, N. F., Ibata, R. A., Irwin, M. J., Chapman, S., Lewis, G. F., Ferguson, A. M. N., Tanvir, N. \& McConnachie, A. W. 2006, MNRAS, 947, in press (arXiv:astro-ph/0607472)

McConnachie, A. W. \& Irwin, M. J. 2006, MNRAS, 365, 1263

Mendes de Oliveira, C. \& Hickson, P. 1994, ApJ, 427, 684

Merritt, D. 1984, ApJ, 276, 26

Michard, R. 1999, A\&AS, 137, 245

Michard, R. 2000, A\&A, 360, 85

Nishiura, S., Murayama, T., Shimada, M., Sato, Y., Nagao, T., Molikawa, K., Taniguchi, Y. \& Sanders, D. B. 2000, AJ, 120, 2355

Mihos, J. C. \& Hernquist, L. 1996 ApJ, 464, 641

Mihos, J. C. 2004, in Clusters of Galaxies: Probes of Cosmological Structure and Galaxy Evolution, Carnegie Observatories Astrophysics Series, J.S. Mulchaey, A. Dressler, and A. Oemler, eds. Cambridge University Press, p. 277

Moore, B., Katz, N., Lake, G., Dressler, A., Oemler, A., Jr. 1996, Nature, 379, 613

Moore, B., Lake, G., \& Katz N. 1998, ApJ, 495, 139

Naab, T., Khochfar, S. \& Burkert, A. 2006a,ApJ, 636,L81

Naab, T., Jesseit, R. \& Burkert, A. 2006b, MNRAS, 372, 839

Oemler, A. 1974, ApJ, 194, 1

Pildis, R. A., Bregman, J. N. \& Schombert, J. M. 1995, AJ, 110, 1498 
Sadler, E. M., 1984, AJ, 89, 23

Schweizer, F. \& Seitzer, P. 1992, AJ, 104, 1039

Tapia, M., Neri, L. \& Roth, M. 1986, RMxAA, 13, 115

Hernández-Toledo, H. M., Avila-Reese, V., Salazar-Contreras, J. R. \& Conselice, C. J. 2006, AJ, 132, 71

Toomre, A. 1977 ARA\&A, 15, 437

van Dokkum, P. G. 2005, AJ, 130, 2647

Verdes-Montenegro, L., Yun, M. S., Williams, B. A., Huchtmeier, W. K., Del Olmo, A. \& Perea, J. 2001, A\&A, 377, 812

Valageas, P., Schaeffer, R. \& Silk, J. 2003, MNRAS, 344, 53

Yoshioka, T., Furuzawa, A., Takahashi, S., Tawara, Y., Sato, S. Yamashita, K. \& Kumai, Y. 2004, Advances in Space Reasearch, 34, 2525

York, D. G., Adelman, J., Anderson, J. E., Anderson, S. F., Annis, J., Bahcall, N. A., Bakken, J. A., Barkhouser, R., Bastian, S., Berman, E., \& 134 coautores 2000, AJ, 120,1579

Zaritsky, D. \& Lo, K. Y. 1986, ApJ, 303, 66

Zepf, S. E., Whitmore, B. C. \& Levison, H. F. 1991, ApJ, 383, 524

Zepf, S. E. \& Whitmore, B. C. 1991, ApJ, 383, 542

Zucker, D. B., Belokurov, V., Evans, N. W., Wilkinson, M. I. and 29 coauthors 2006, ApJ, 643, 103 

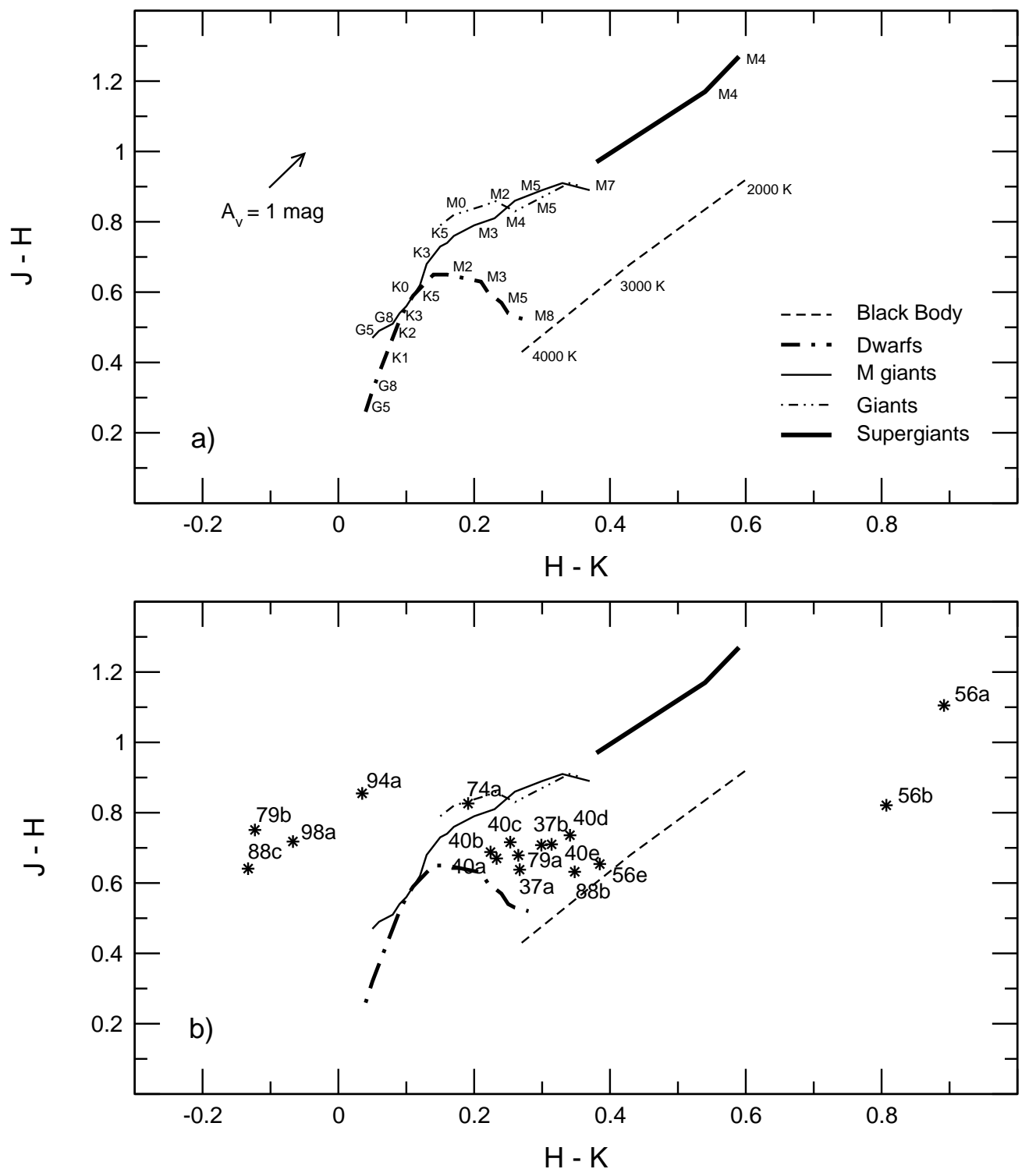

Fig. 1.- a) Color-color diagram, using 2MASS, of stars having different spectral types. b) The colors of the galaxies in our sample are compared to those of the stars. Except for HCG 56a and HCG 56b, the comparison shows that the colors of the galaxies can be explain by a mixture of $\mathrm{M}$ dwarf and $\mathrm{M}$ and $\mathrm{K}$ giant stars. 


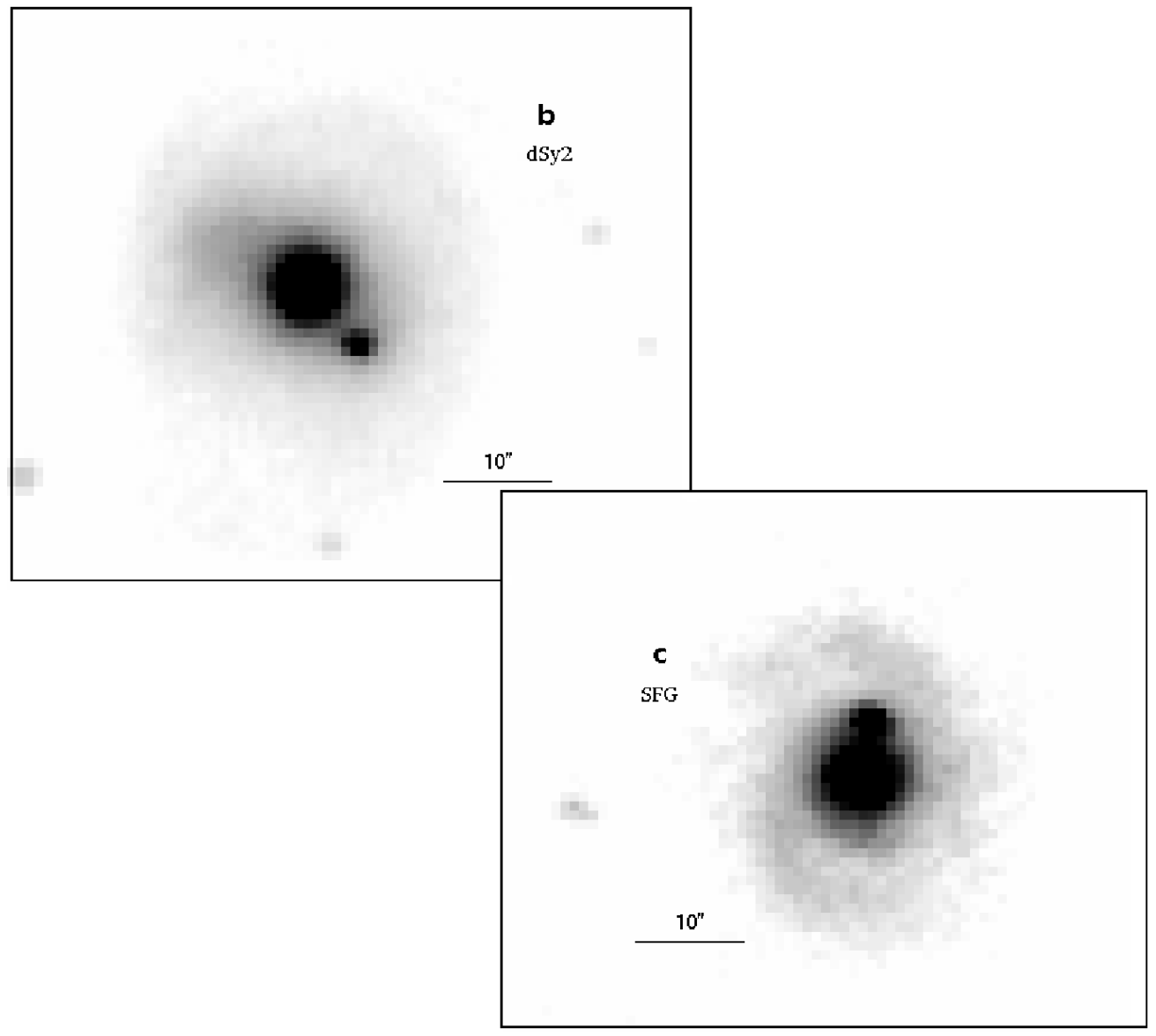

Fig. 2.- Images in J band (in negative) of HCG 88b and HCG88c. The north is up and east is to the left. The fainter grey structures shown in these images have a $\mathrm{S} / \mathrm{N}$ of 29 and 17, respectively in HCG 88b and HCG88c. 


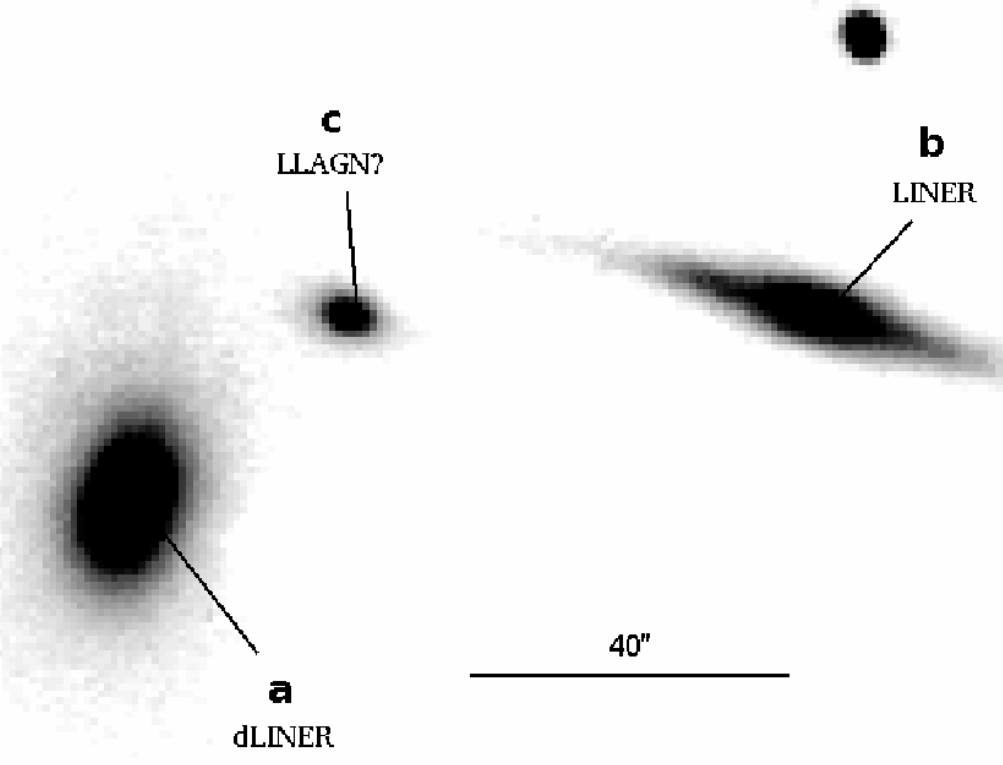

Fig. 3.- Same as Figure 2 for HCG 37. The fainter grey structures shown in this image have a mean $\mathrm{S} / \mathrm{N}$ of 13 . 

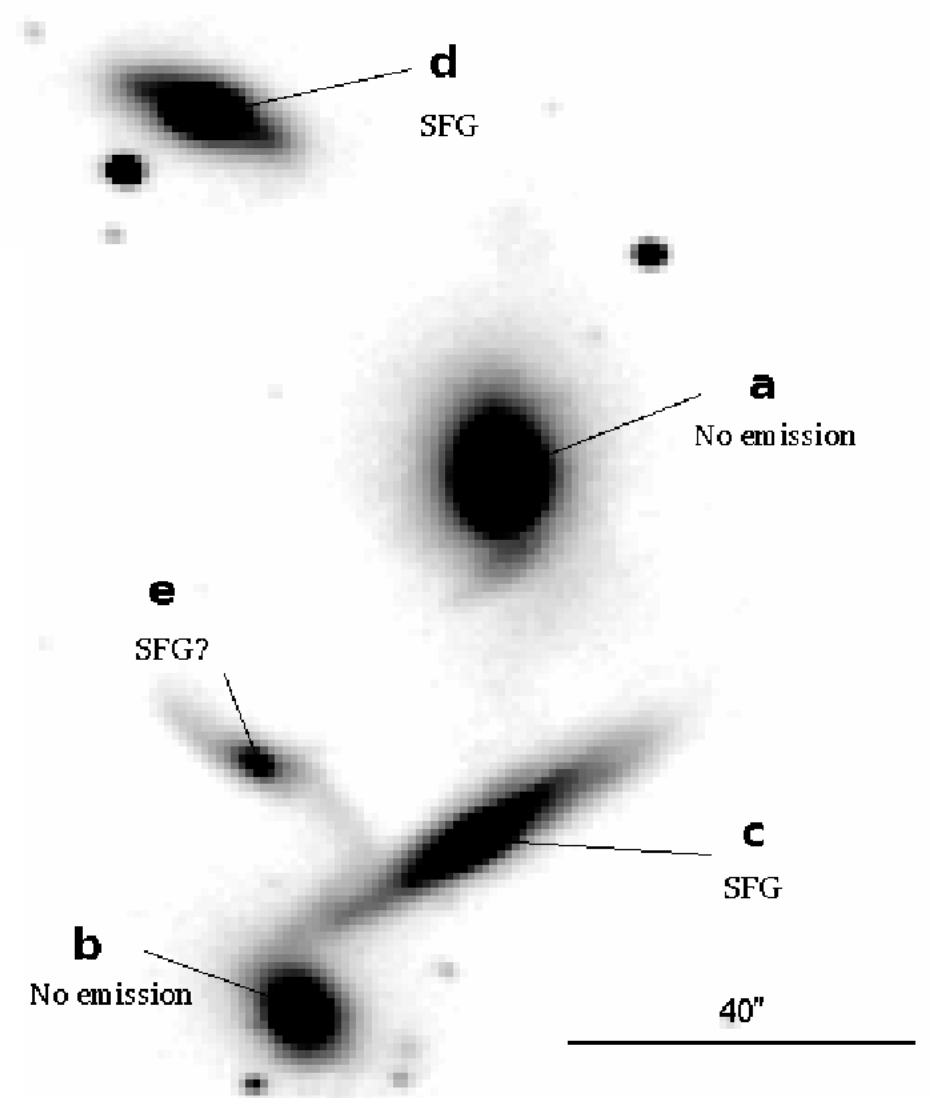

Fig. 4.- Same as Figure 2 for HCG 40. The fainter grey structures shown in this image have a mean $\mathrm{S} / \mathrm{N}$ of 25 . 

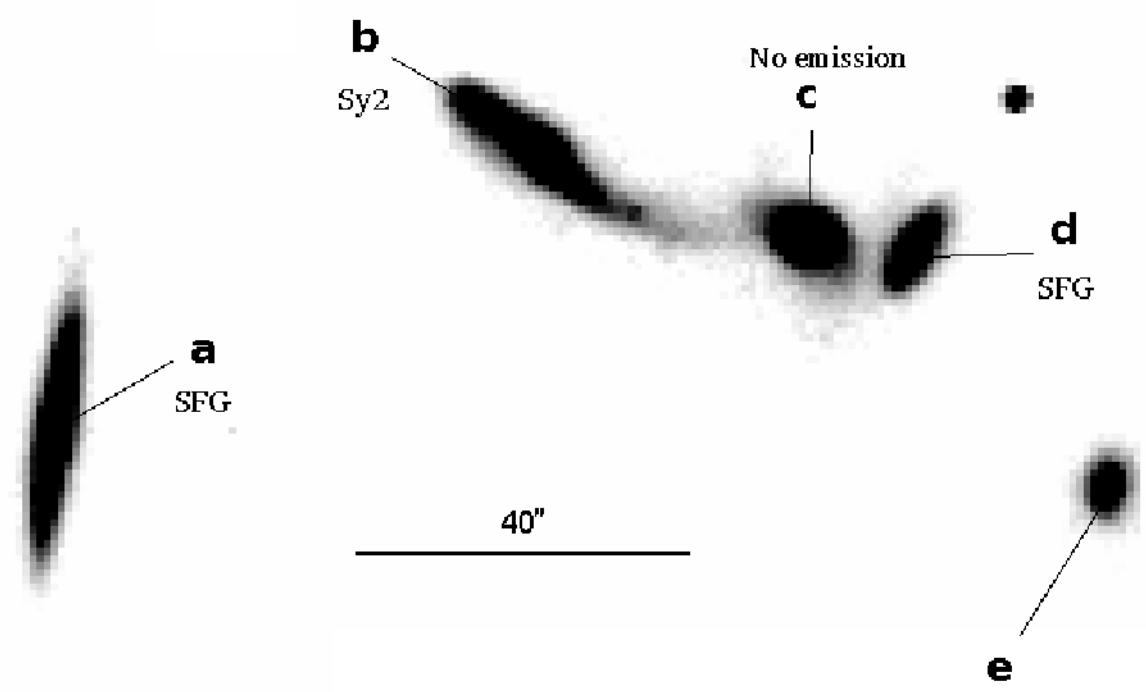

SFG?

Fig. 5.- Same as Figure 2 for HCG 56. The fainter grey structures shown in this image have a mean $\mathrm{S} / \mathrm{N}$ of 4 . 


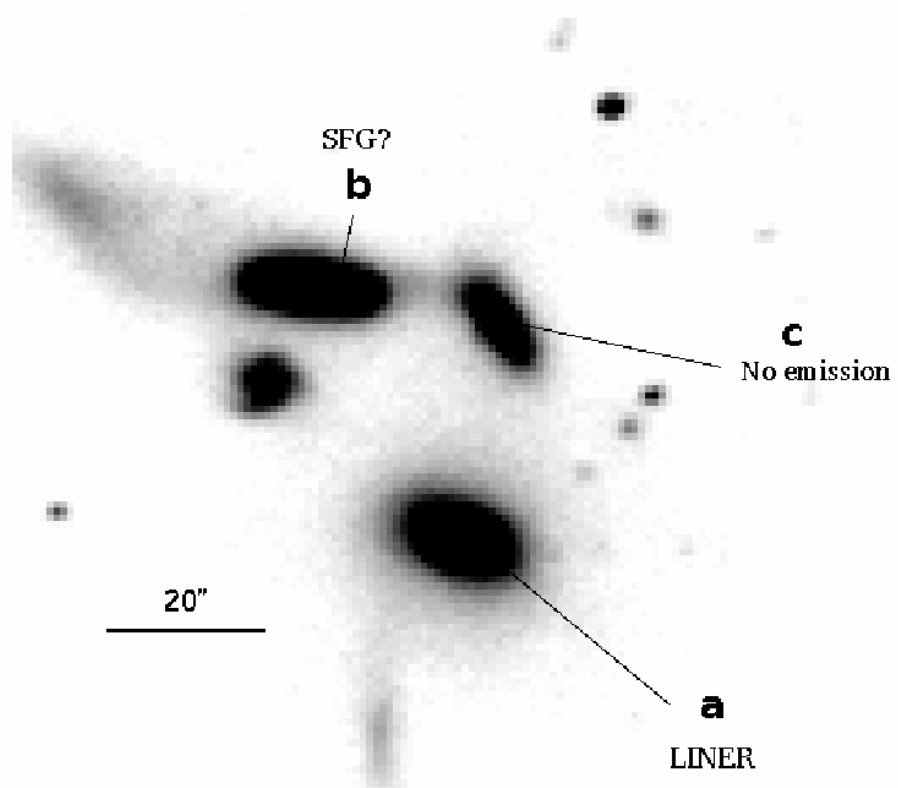

Fig. 6.- Same as Figure 2 for HCG 79. The fainter grey structures shown in this image have a mean $\mathrm{S} / \mathrm{N}$ of 12 . 


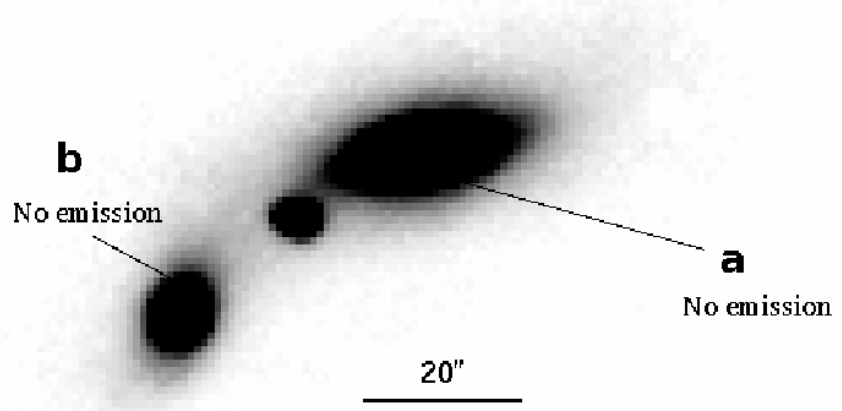

Fig. 7.- Same as Figure 2 for HCG 98. The non-identified objects in the center is a foreground star. The fainter grey structures shown in this image have a mean $\mathrm{S} / \mathrm{N}$ of 20 . 


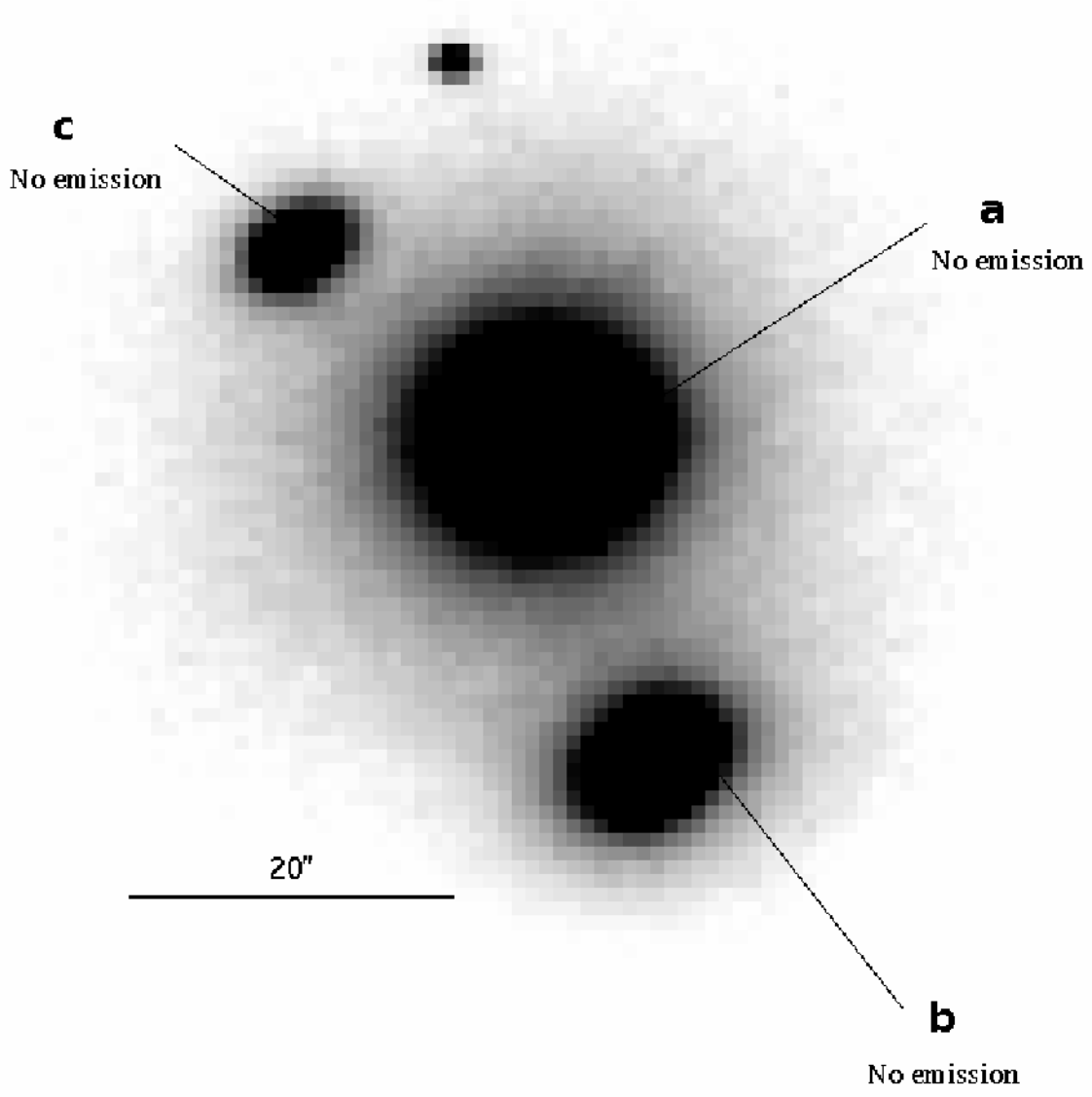

Fig. 8.- Same as Figure 2 for HCG 74. The fainter grey structures shown in this image have a mean $\mathrm{S} / \mathrm{N}$ of 27 . 


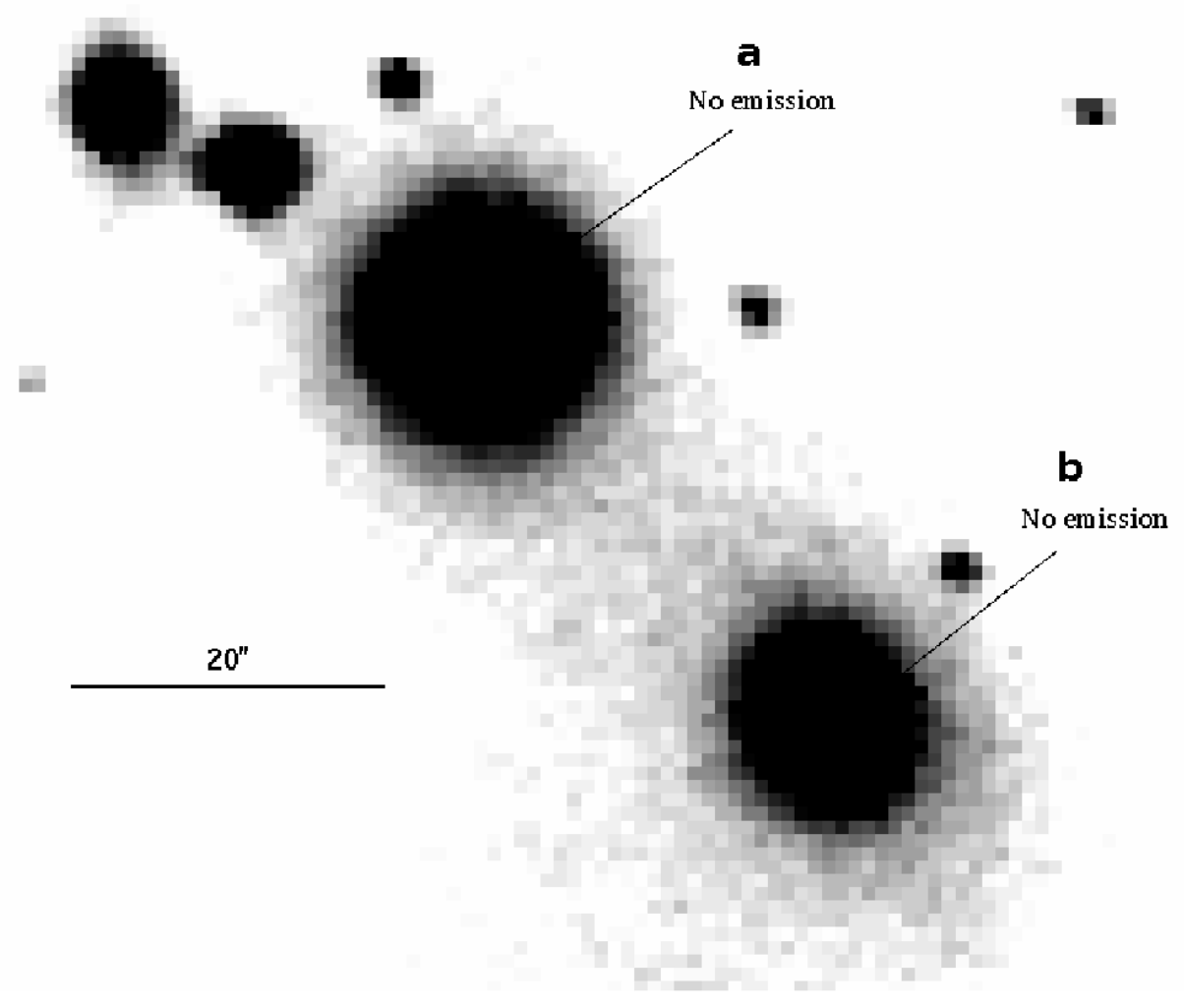

Fig. 9.- Same as Figure 2 for HCG 94. The fainter grey structures shown in this image have a mean $\mathrm{S} / \mathrm{N}$ of 2 . 

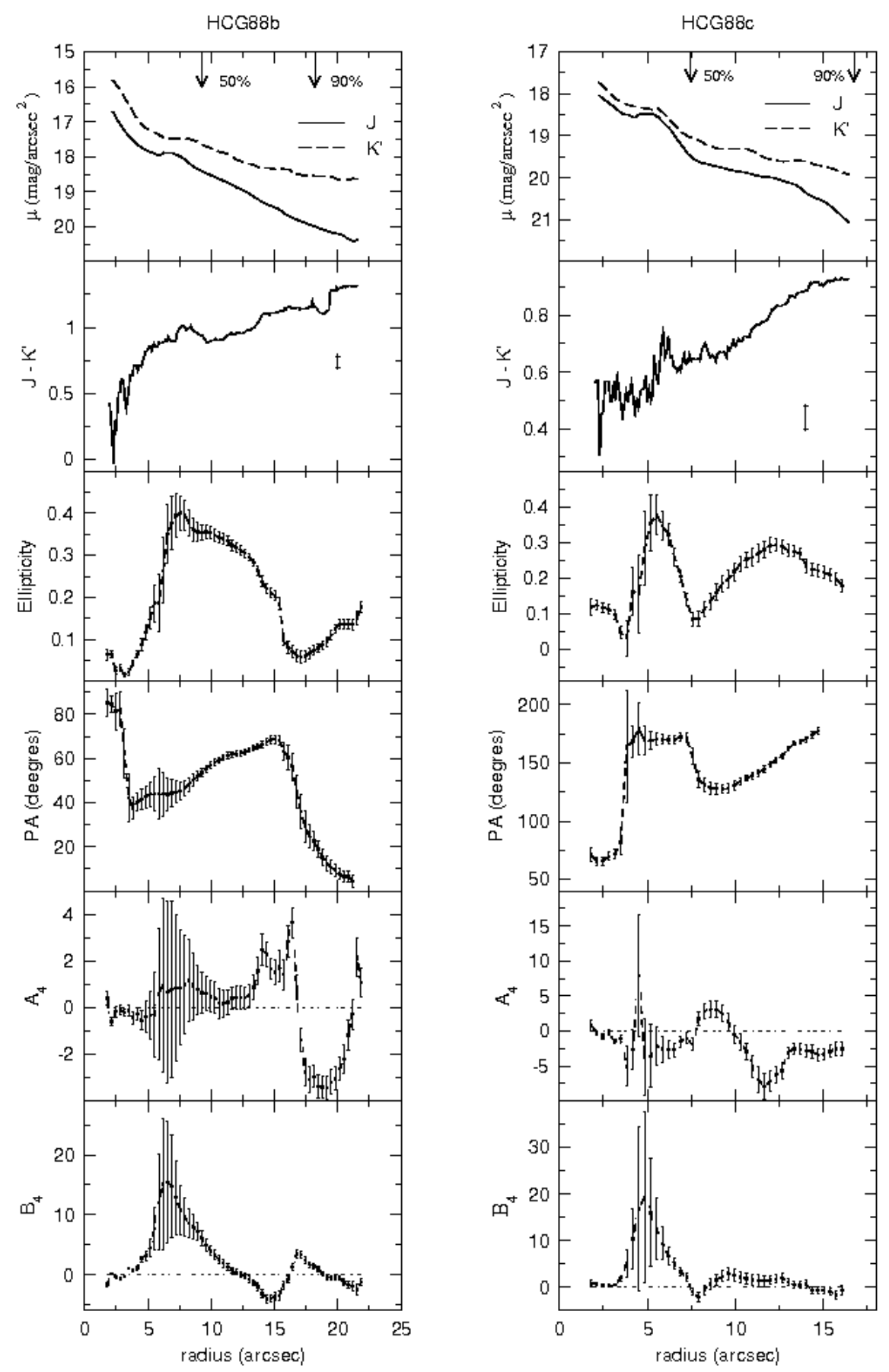

Fig. 10.- Results of the fitted ellipses analysis. The graphics show, from top to bottom, the surface brightness profiles ( $\mathrm{J}$ and $\mathrm{K}$ '), the color profile (with typical error bar) and variations with radius of the ellipticity, position angle, and the fourth order Fourier coefficients. The two arrows in the upper graph indicate the radii containing $50 \%$ and $90 \%$ of the light. 

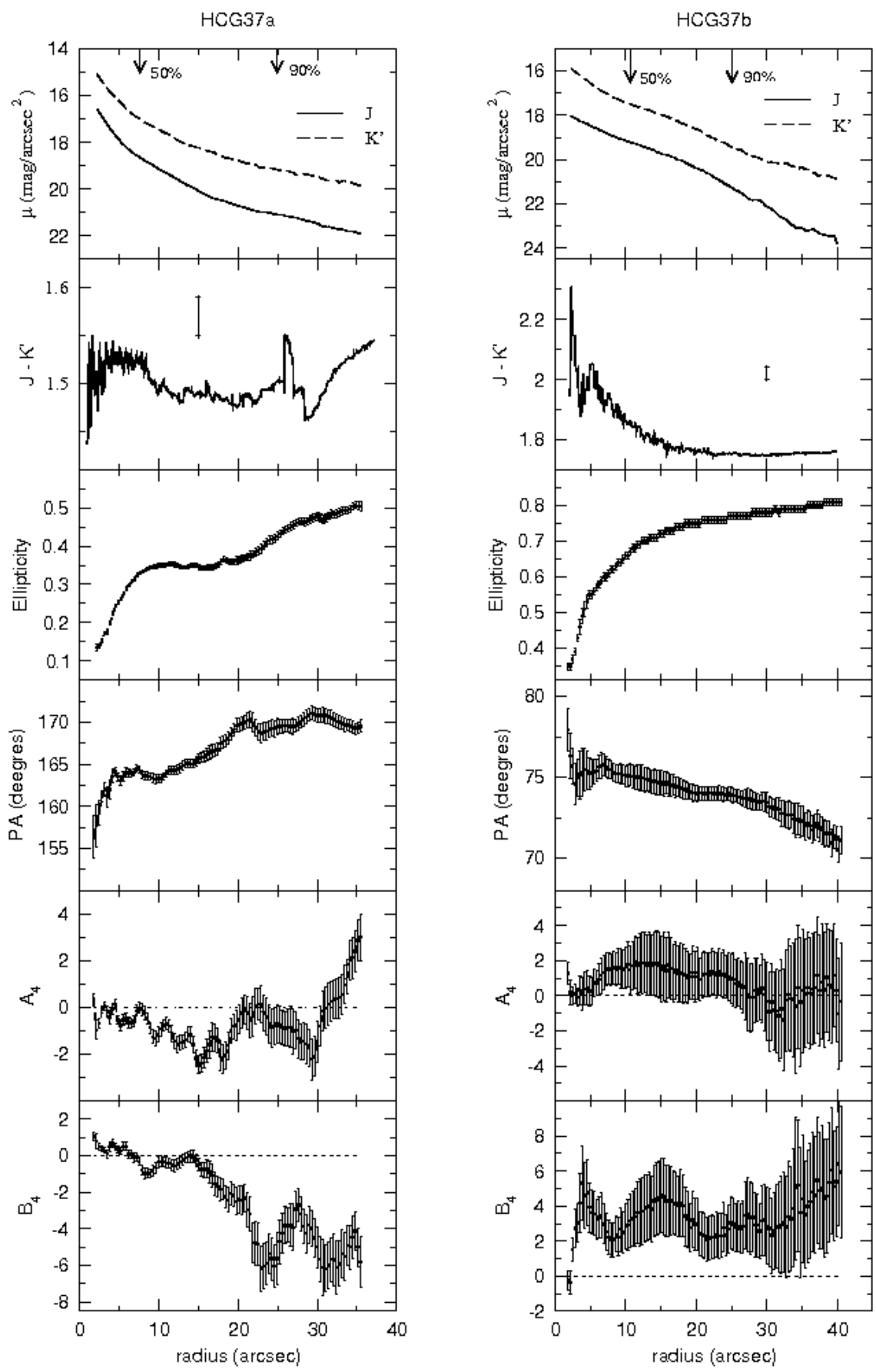

Fig. 11. - Same as figure 10. 

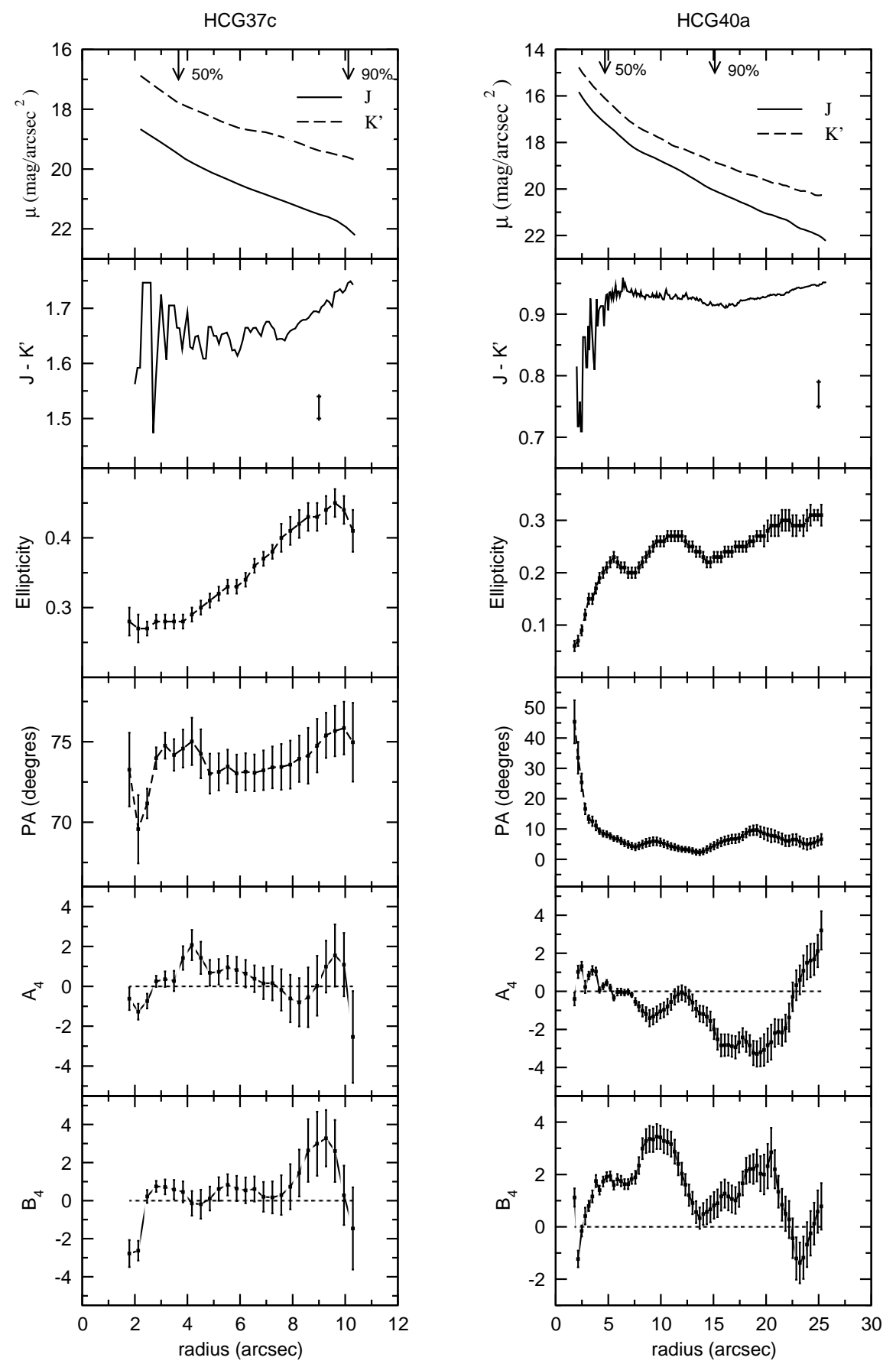

Fig. 12.- Same as figure 10. 

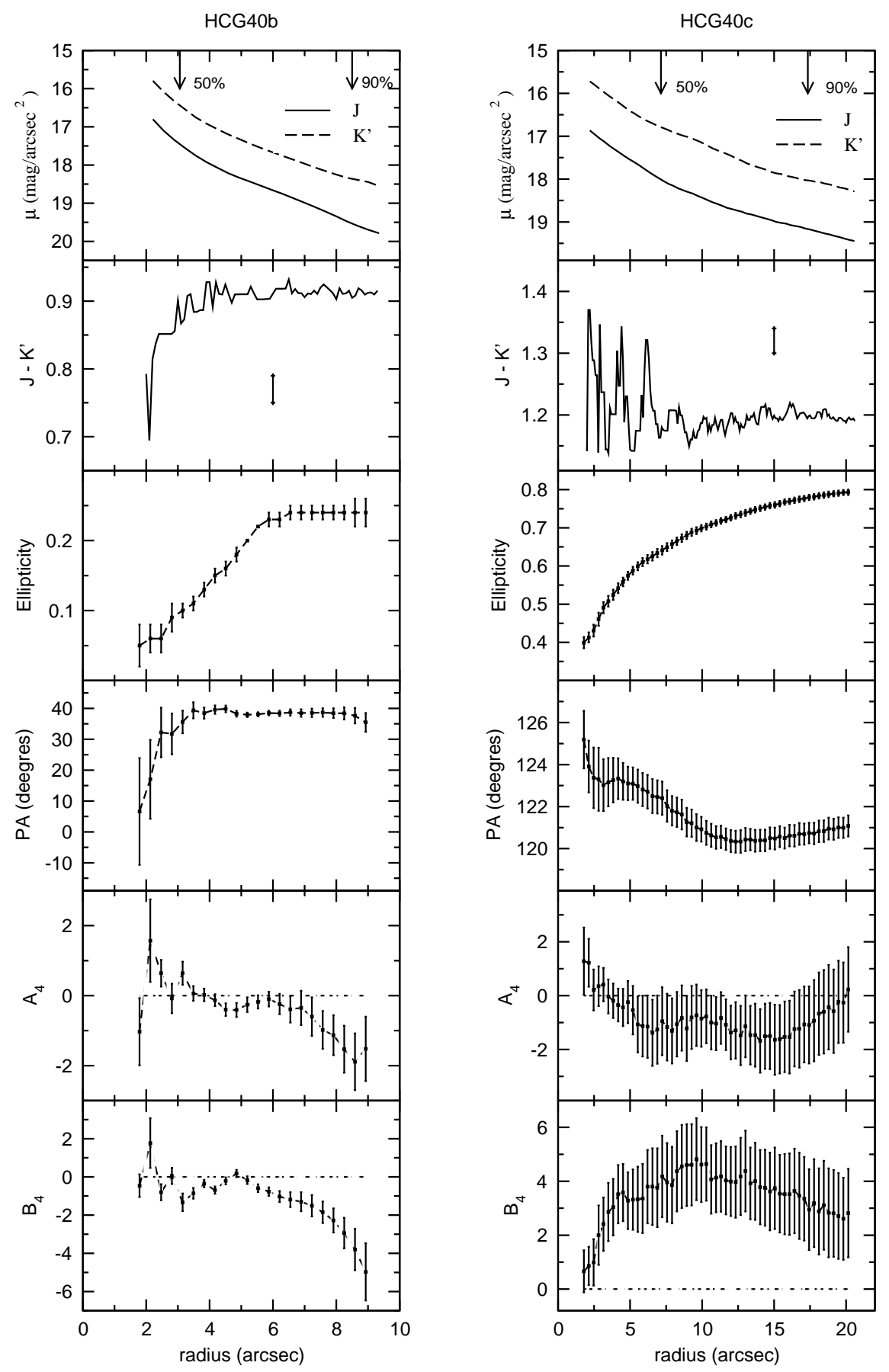

Fig. 13.- Same as figure 10. 

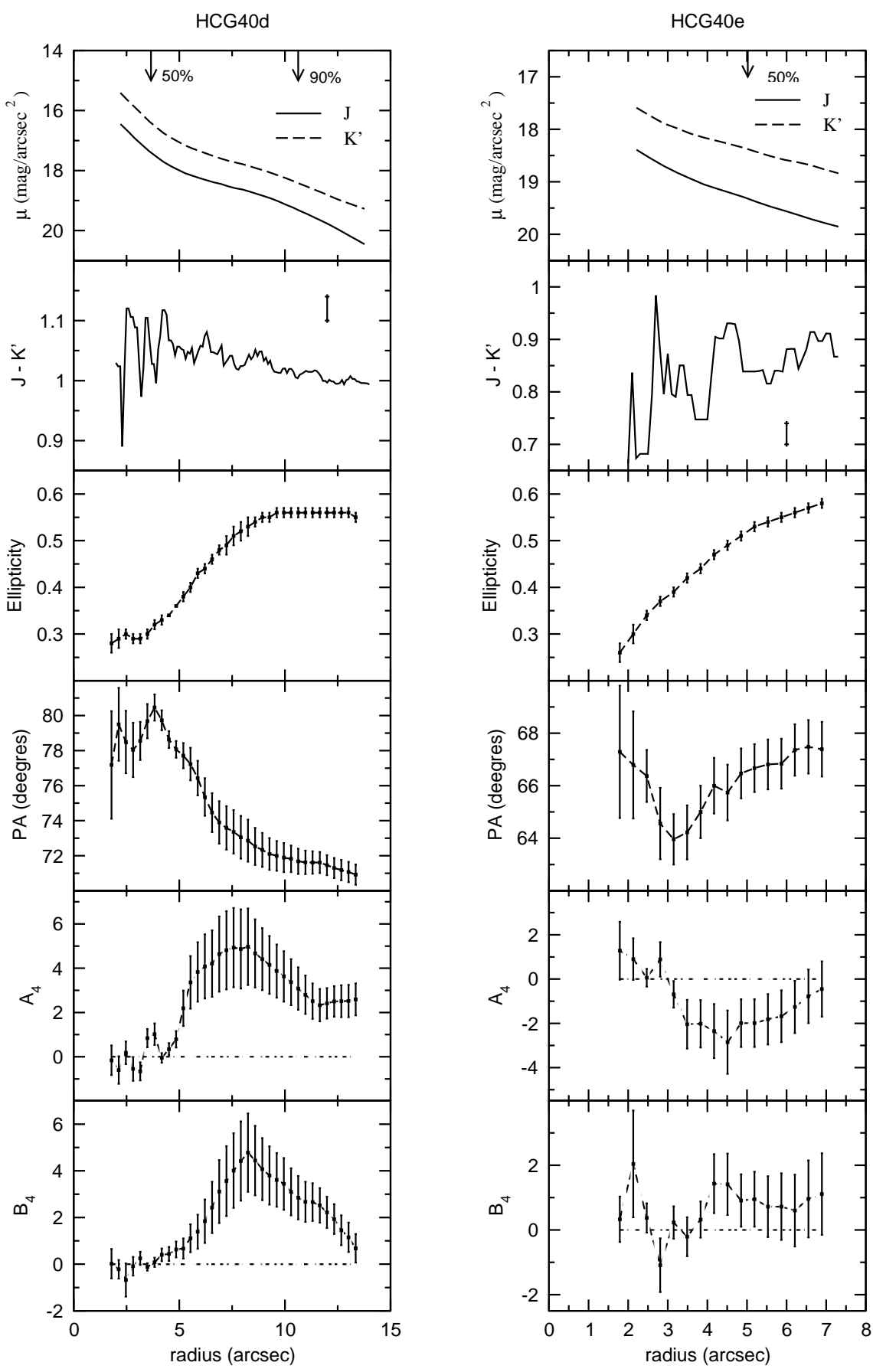

Fig. 14.- Same as figure 10. 

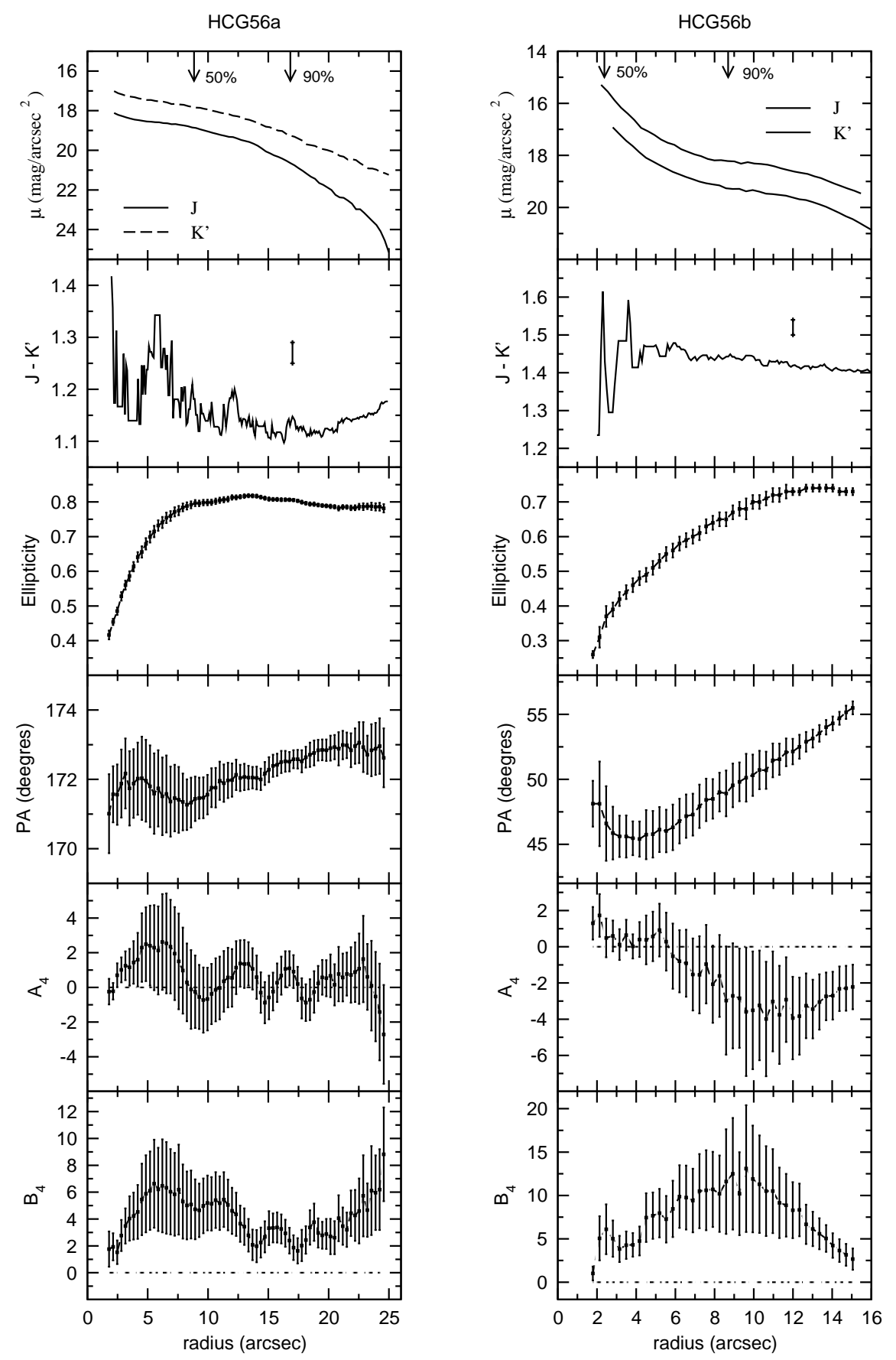

Fig. 15. - Same as figure 10. 

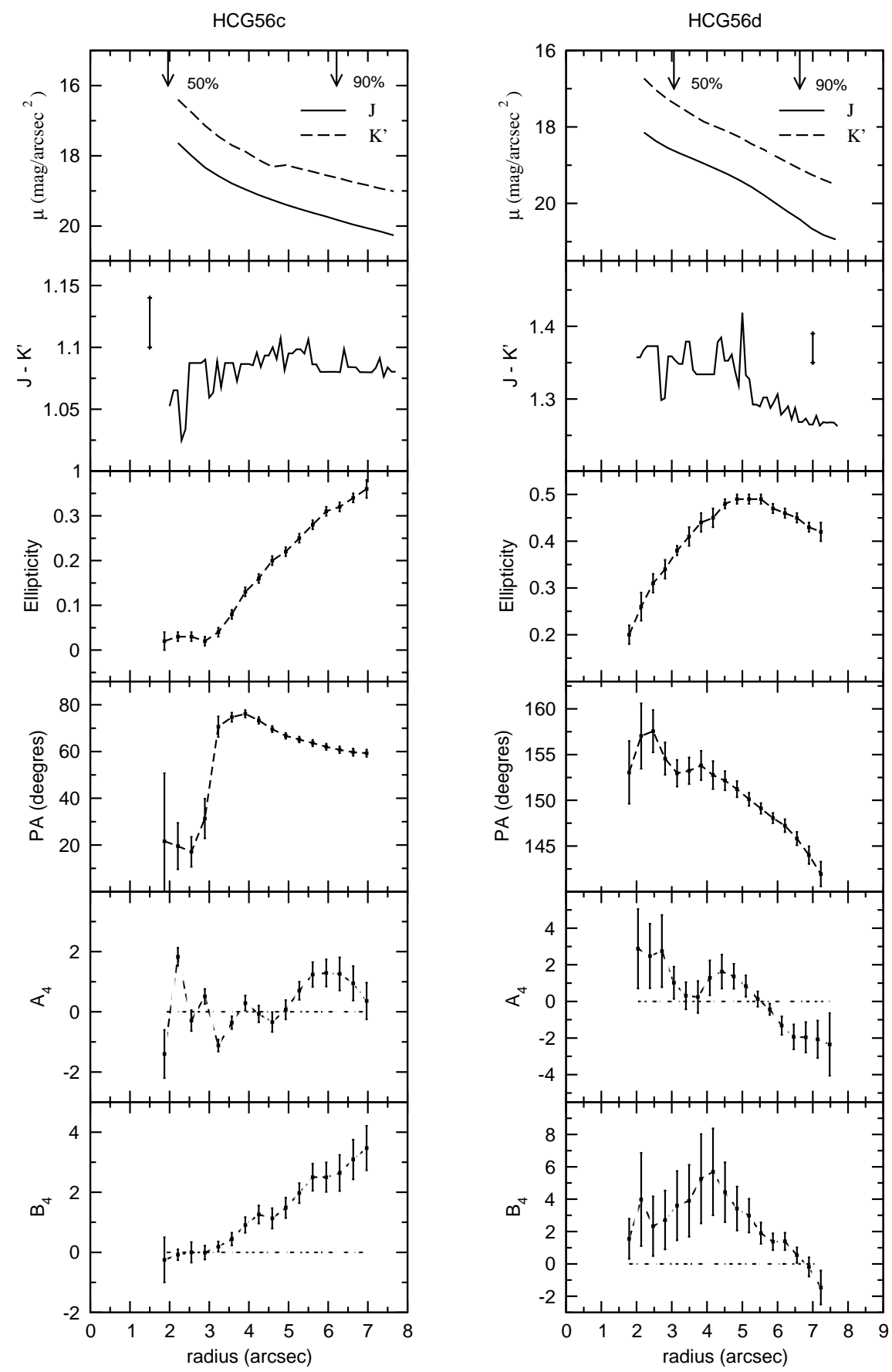

Fig. 16.- Same as figure 10. 

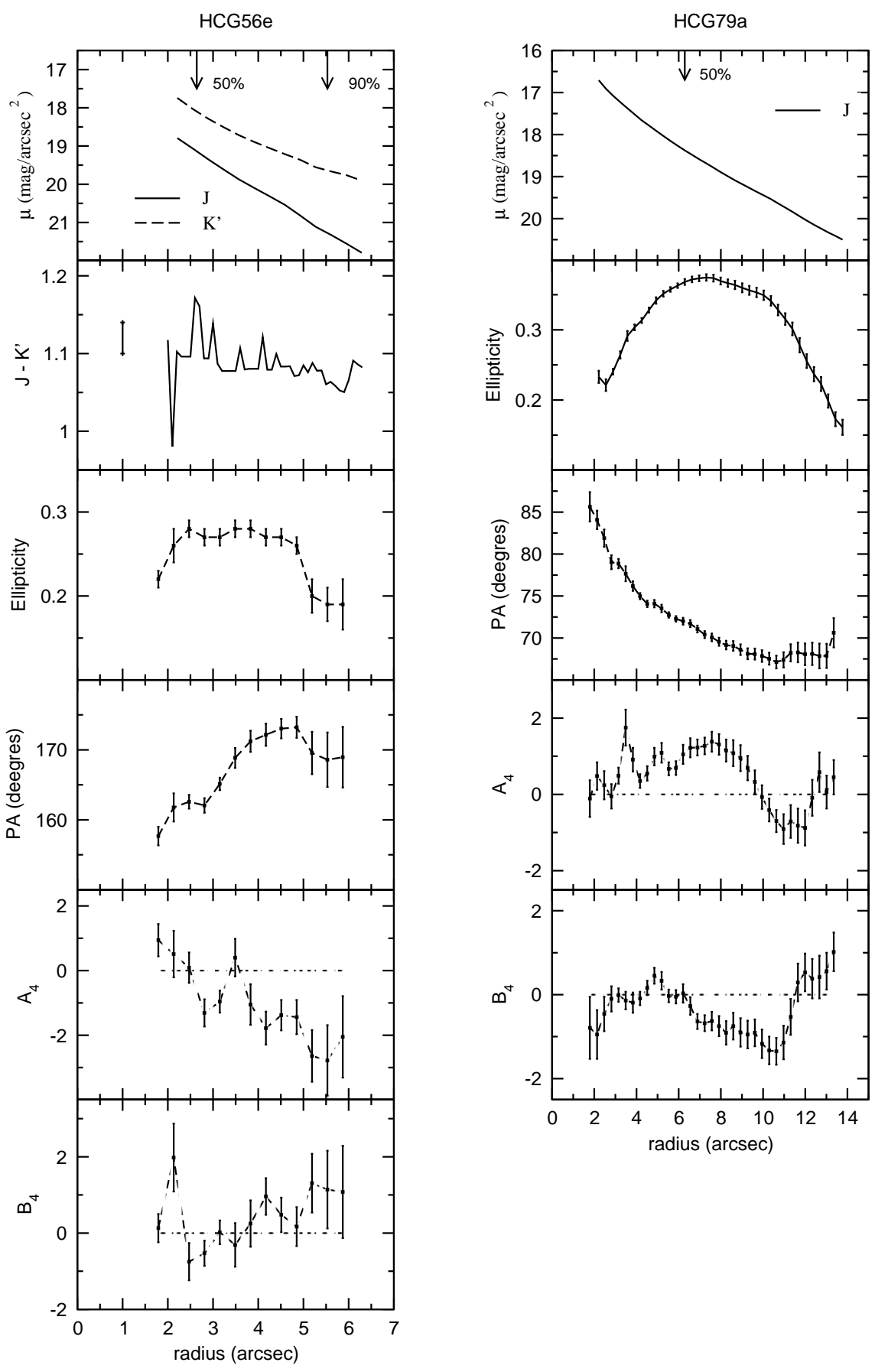

Fig. 17.- Same as figure 10 for HCG 56e. The K' image is missing in the case of HCG 79a. 

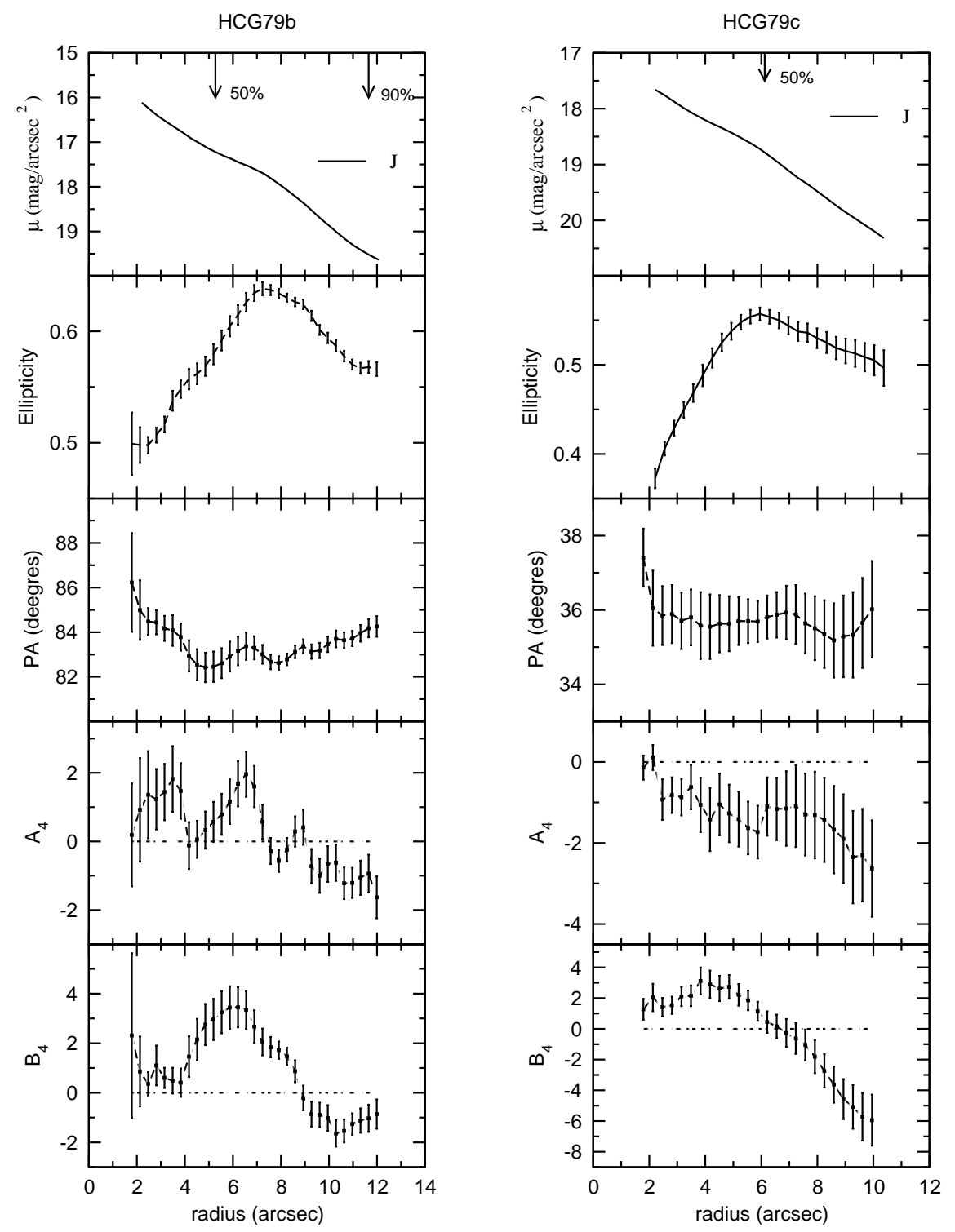

Fig. 18. - Same as figure 10 , except that the $\mathrm{K}^{\prime}$ image is missing. 

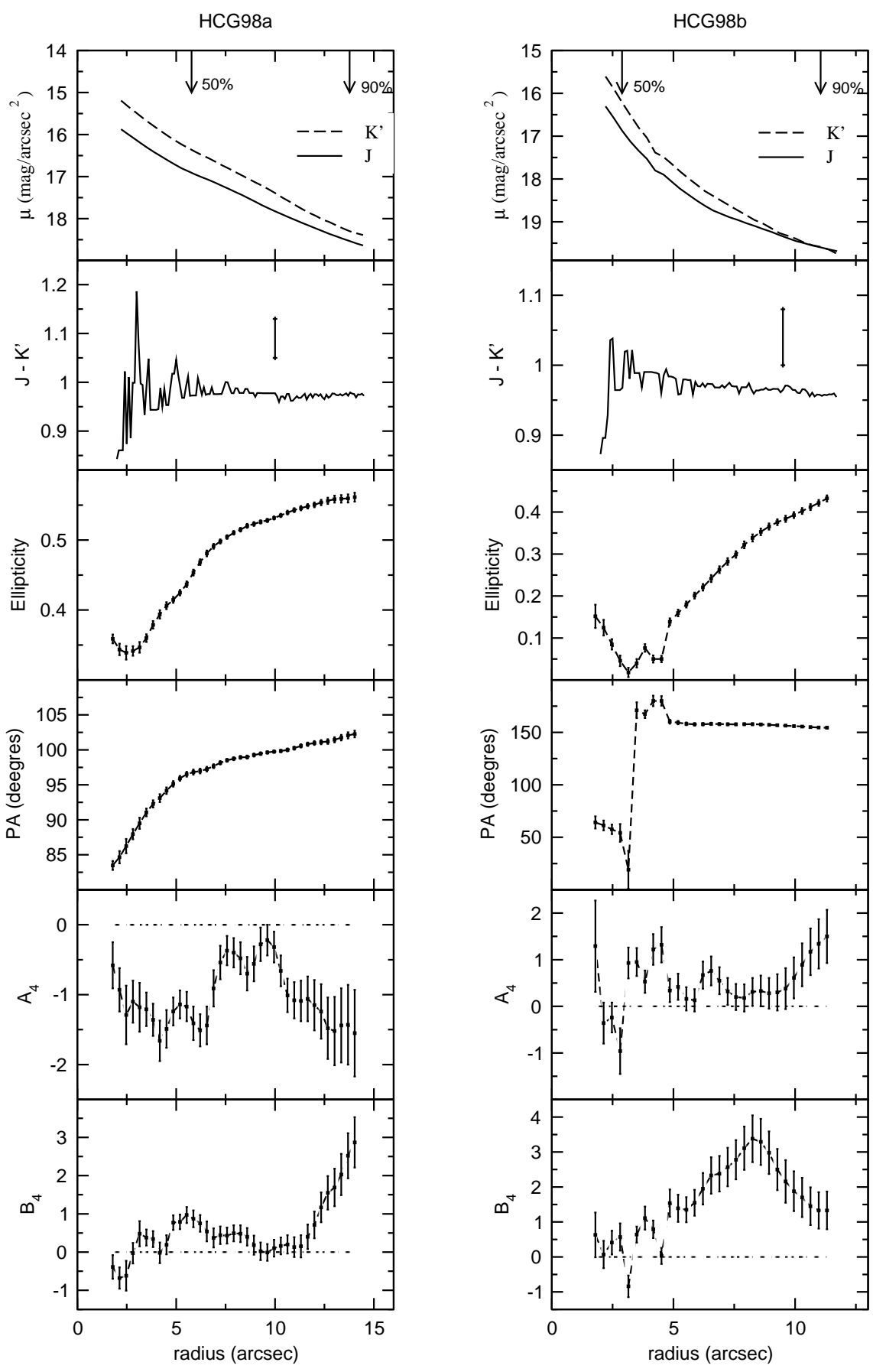

Fig. 19.- Same as figure 10. 

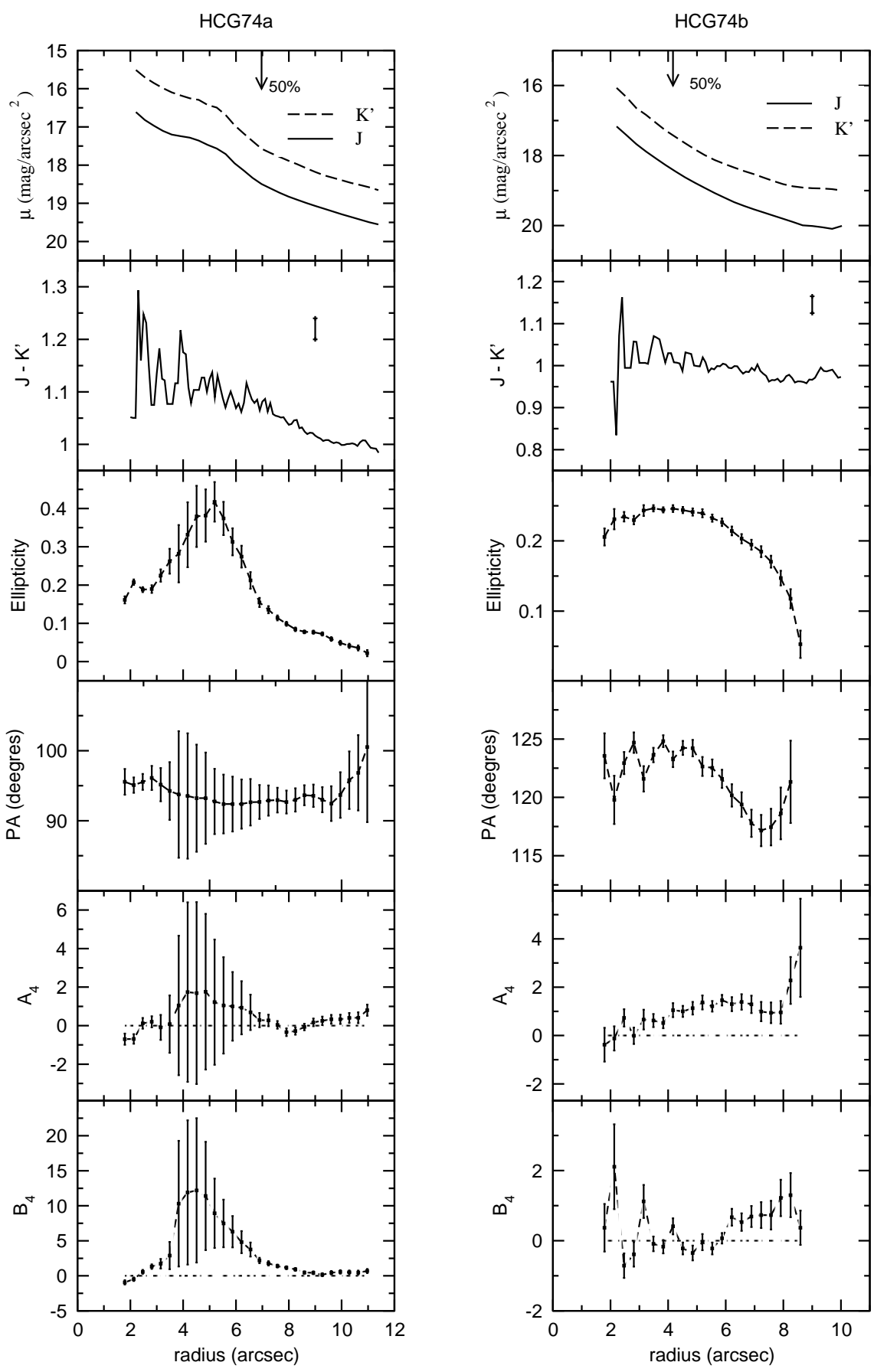

Fig. 20.- Same as figure 10. 

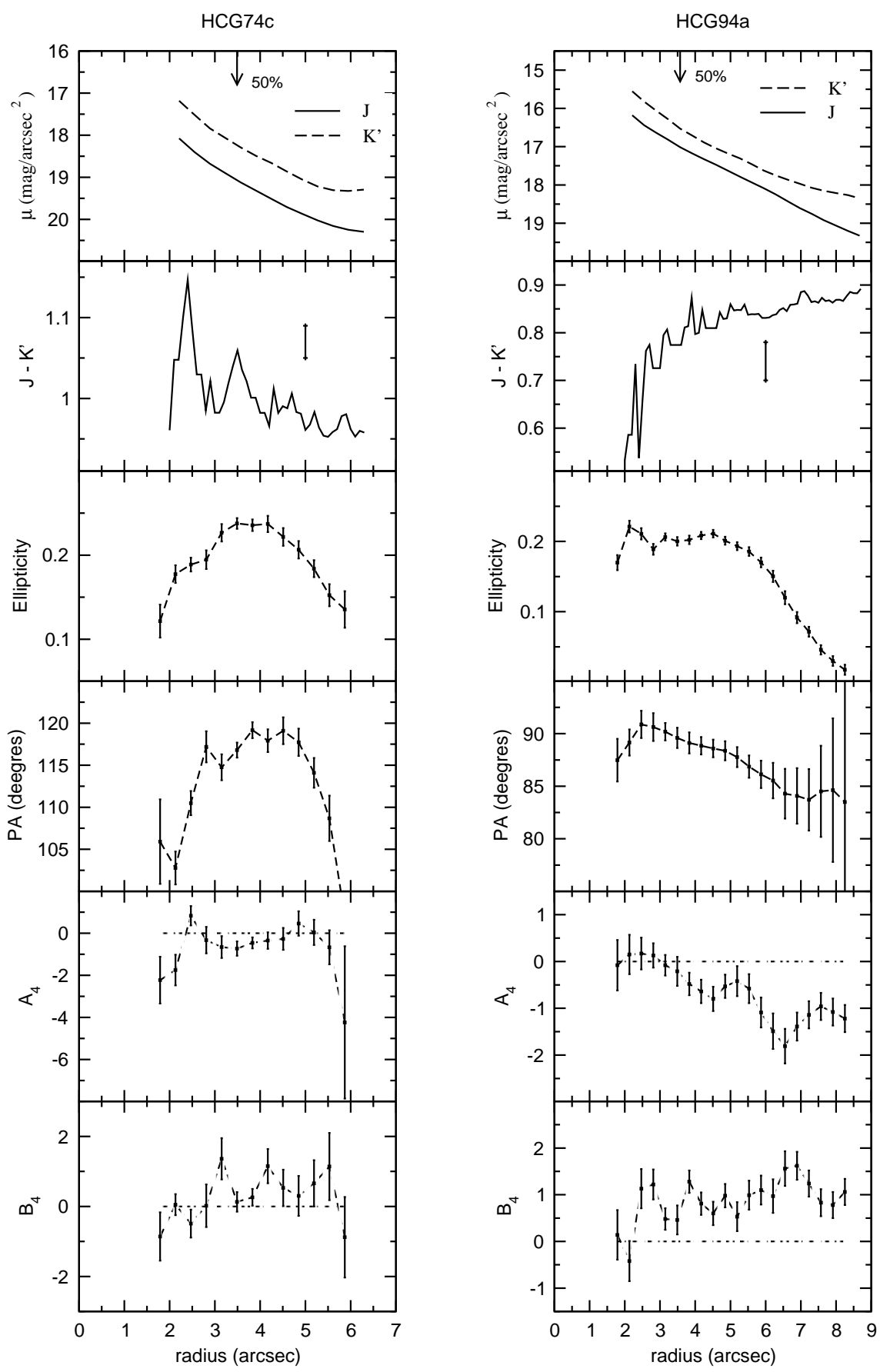

Fig. 21. - Same as figure 10. 


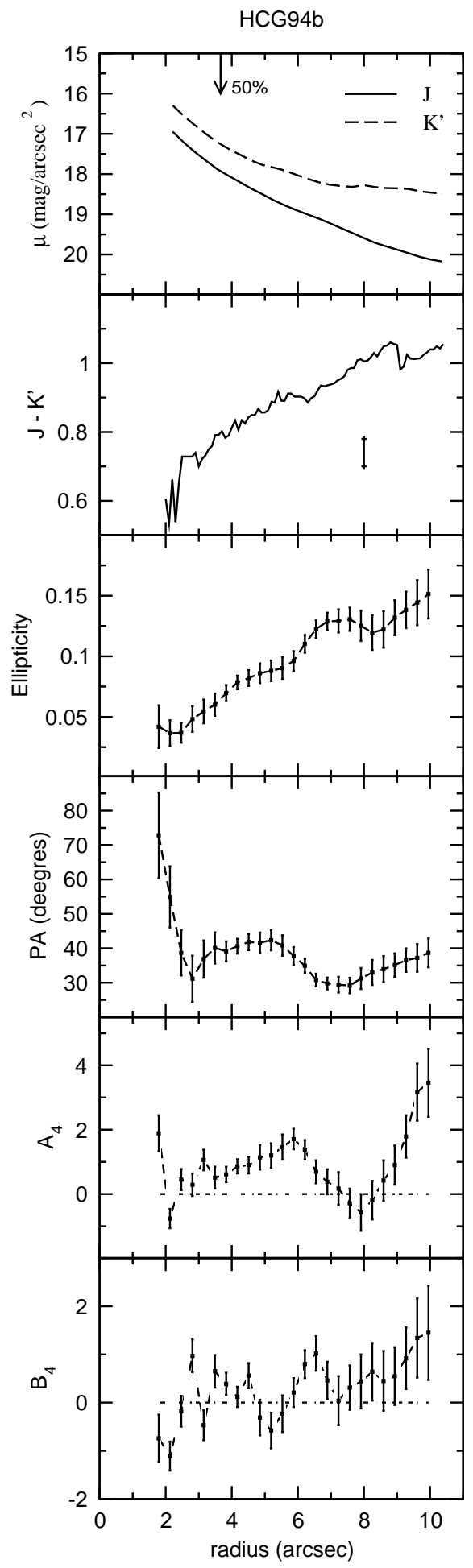

Fig. 22.- Same as figure 10. 

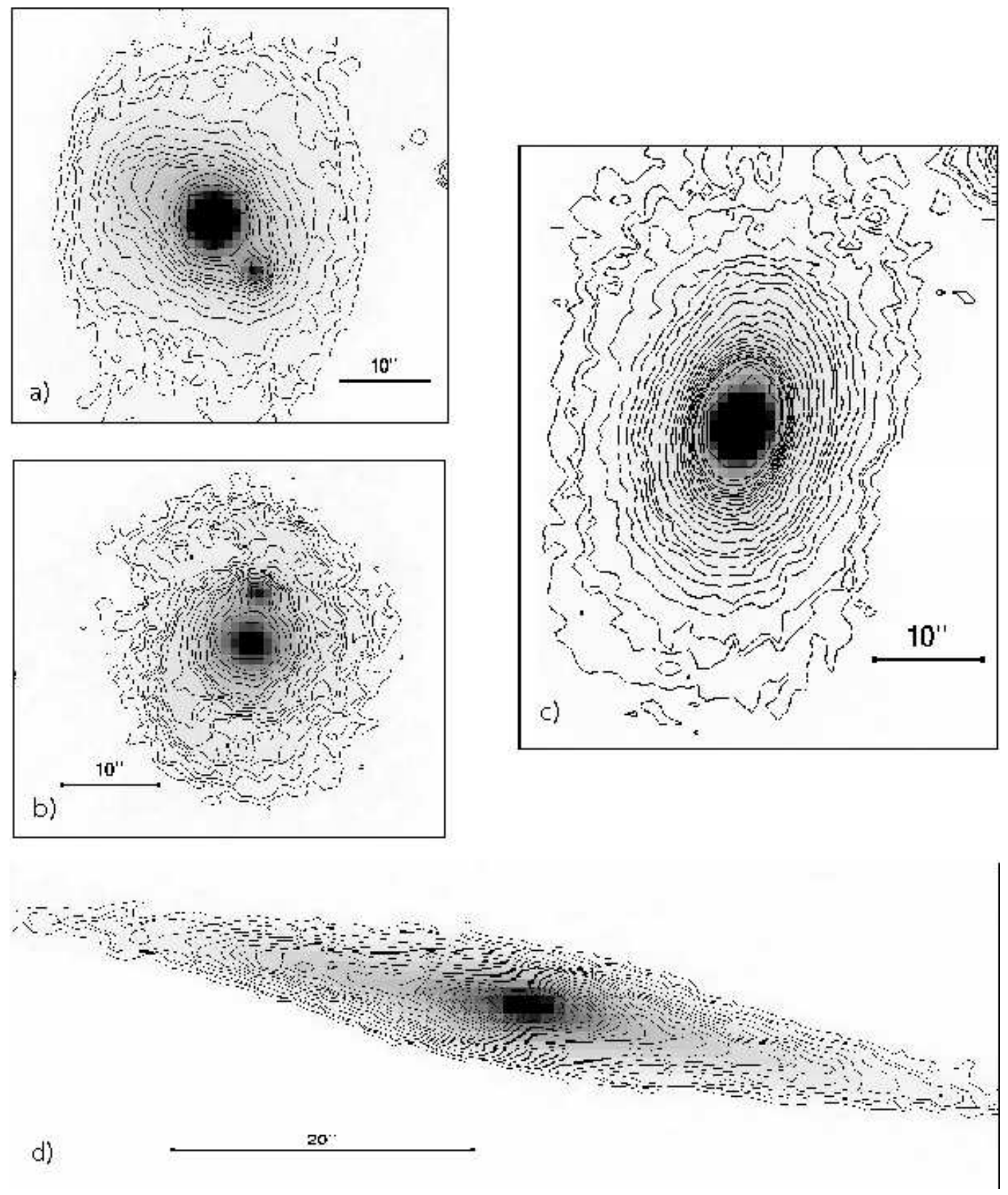

Fig. 23. - Photometrically uncalibrated isophotes (units in ADU), overlayed on the negative $\mathrm{J}$ images of the galaxies in our sample. The levels decrease with radius by $5 \%$ (compared to the inner isophote). As usual, north is up and east to the left. The galaxies are: a) HCG 88b, b) HCG 88c, c) HCG 37a, and d) HCG 37b. 

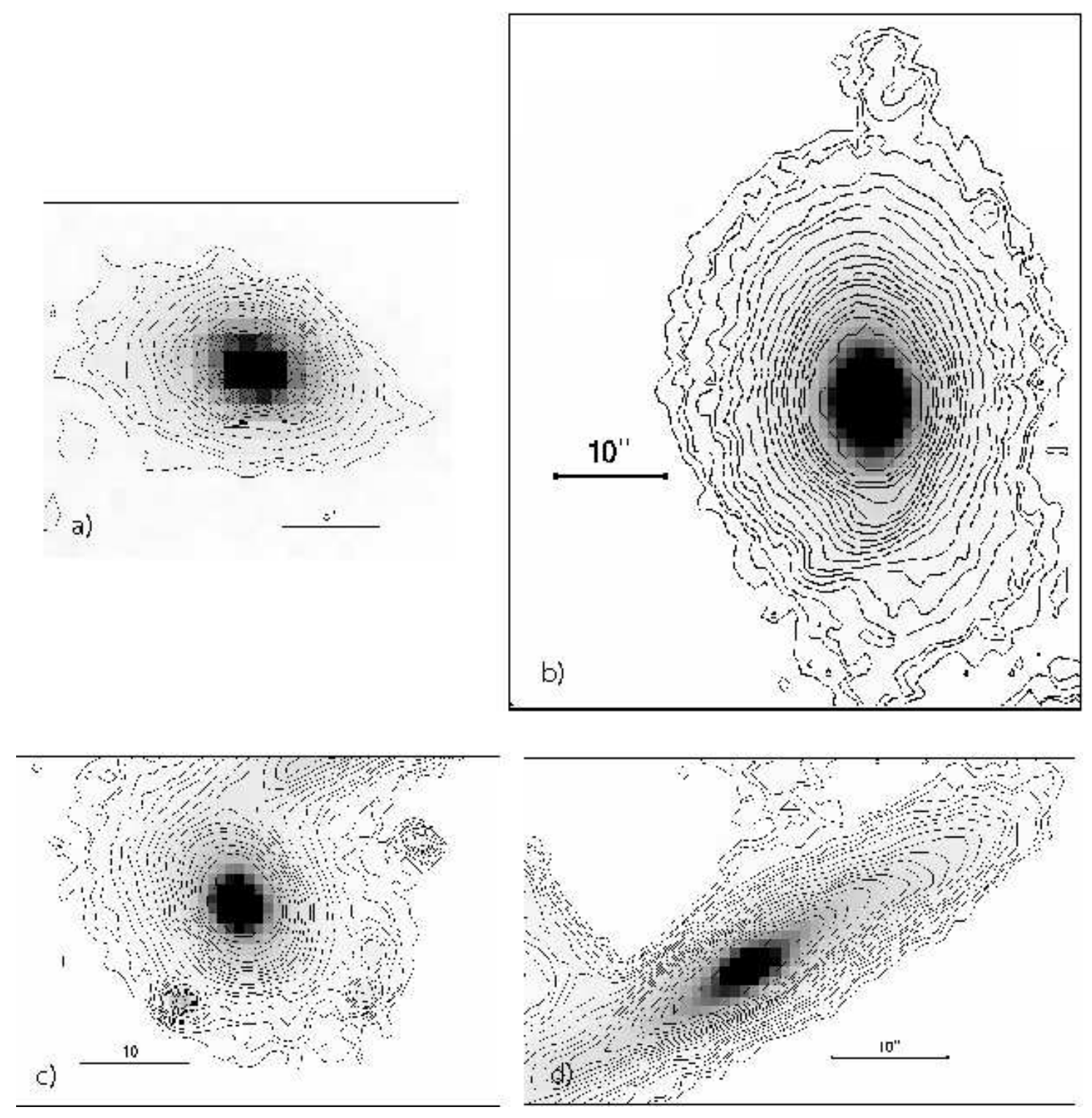

Fig. 24.- Same as Figure 23 for a) HCG 37c, b) HCG 40a, c) HCG 40b, and d) HCG 40c. 

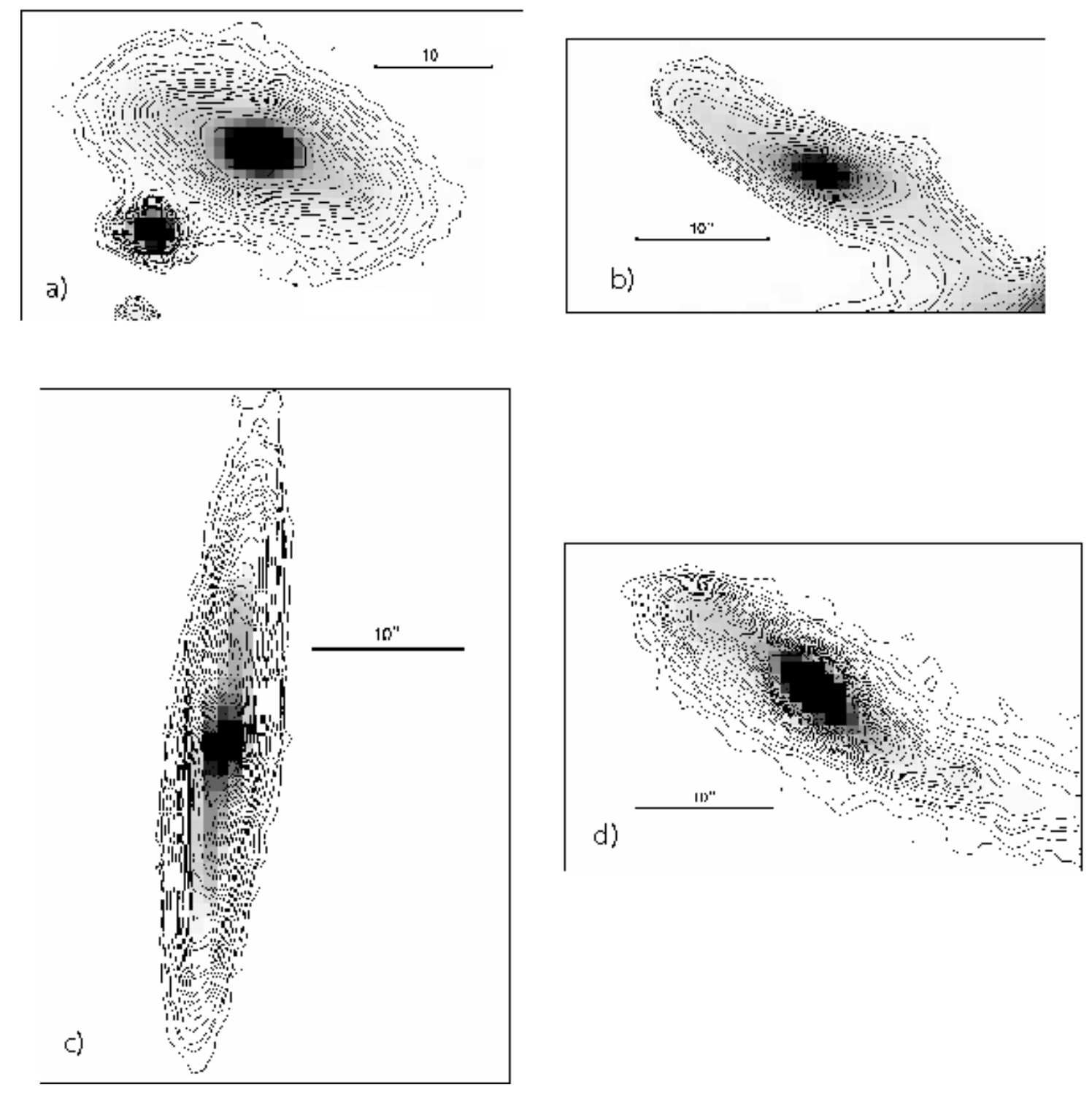

Fig. 25.- Same as Figure 23 for a) HCG 40d, b) HCG 40e, c) HCG 56a, and d) HCG 56b. 

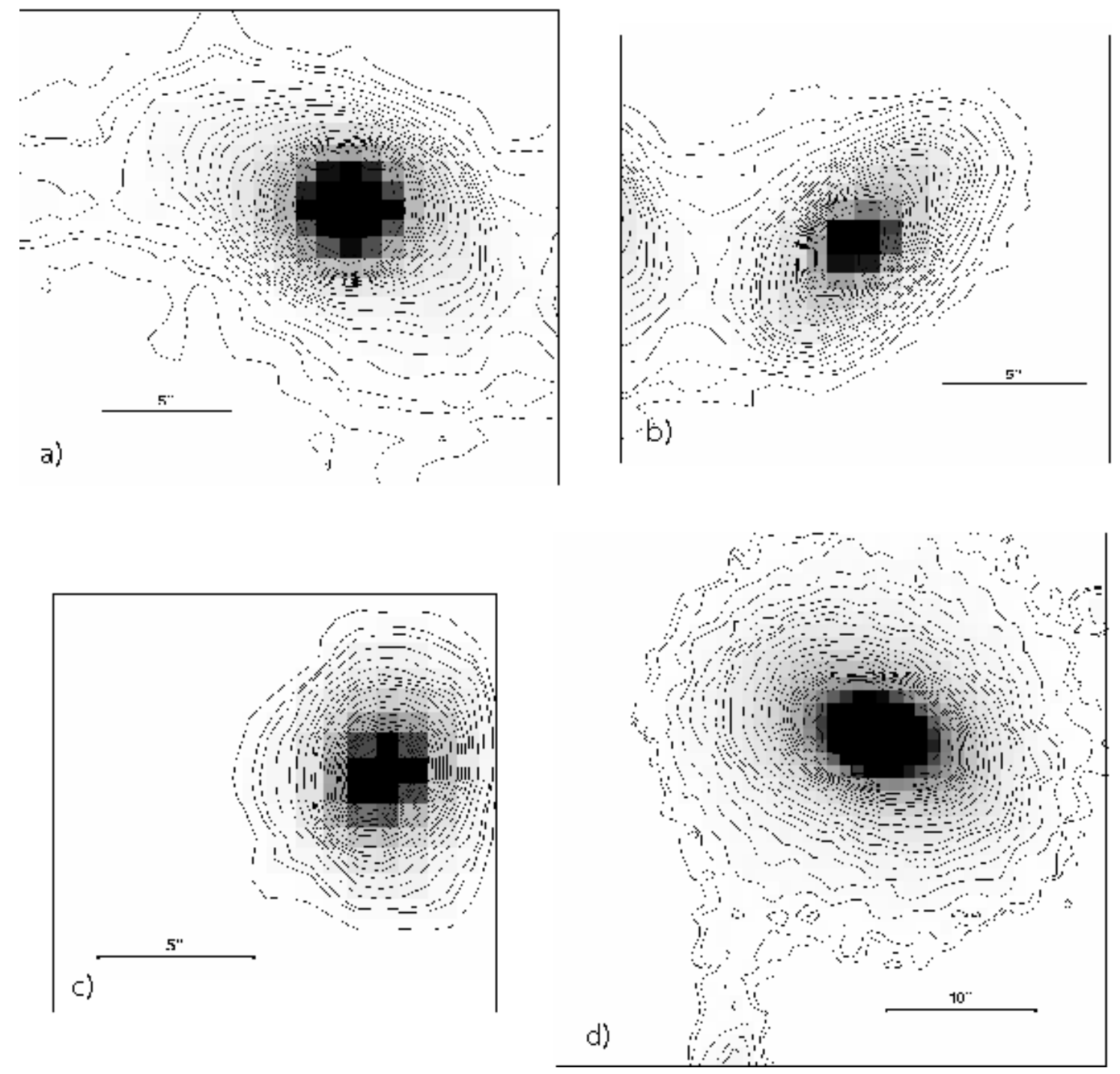

Fig. 26.- Same as Figure 23 for a) HCG 56c, b) HCG 56d, c) HCG 56e, and d) HCG 79a. 

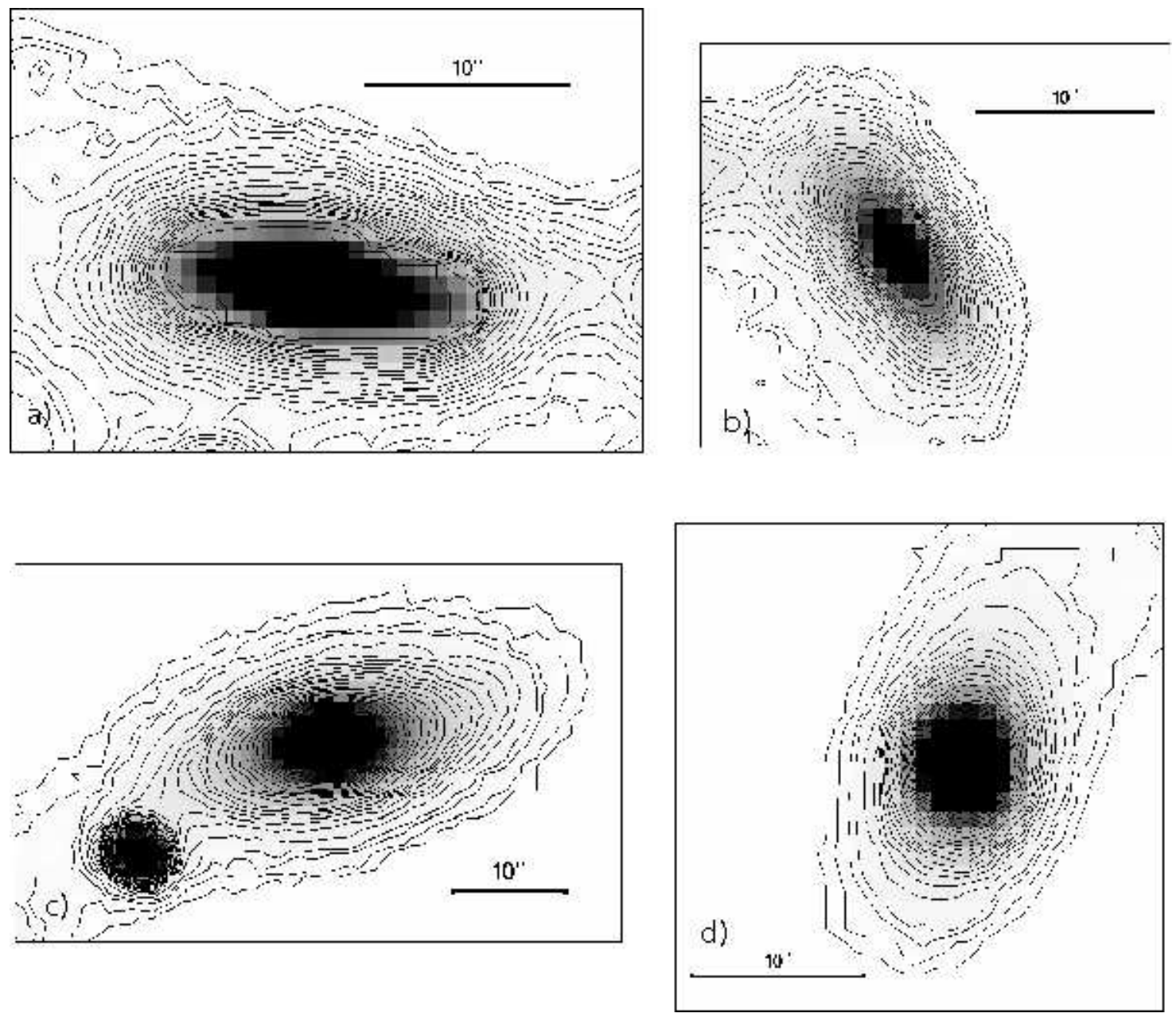

Fig. 27.- Same as Figure 23 for a) HCG 79b, b) HCG 79c, c) HCG 98a, and d) HCG 98b. 

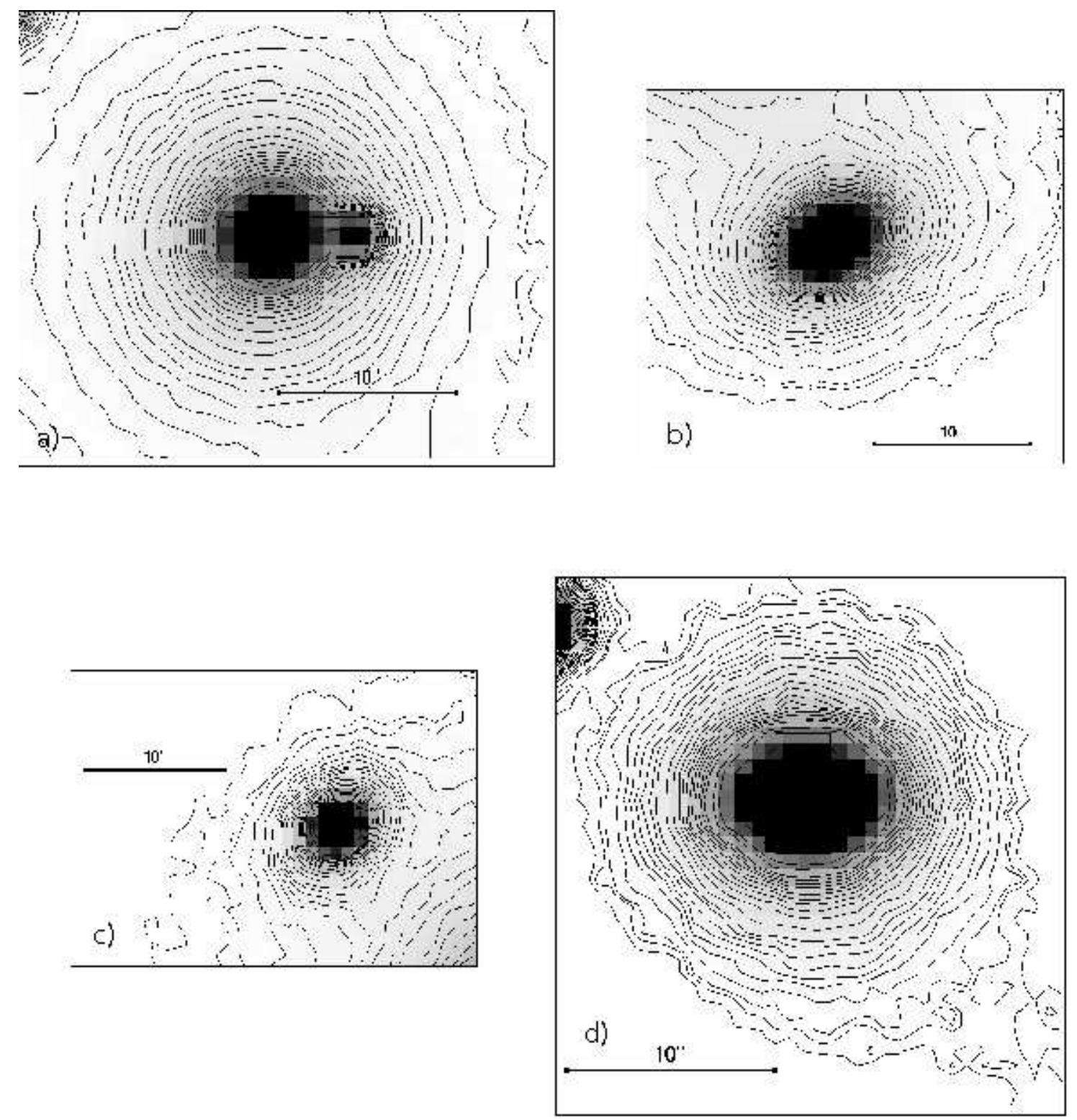

Fig. 28. - Same as Figure 23 for a) HCG 74a, b) HCG 74b, c) HCG 74c, and d) HCG 94a. 


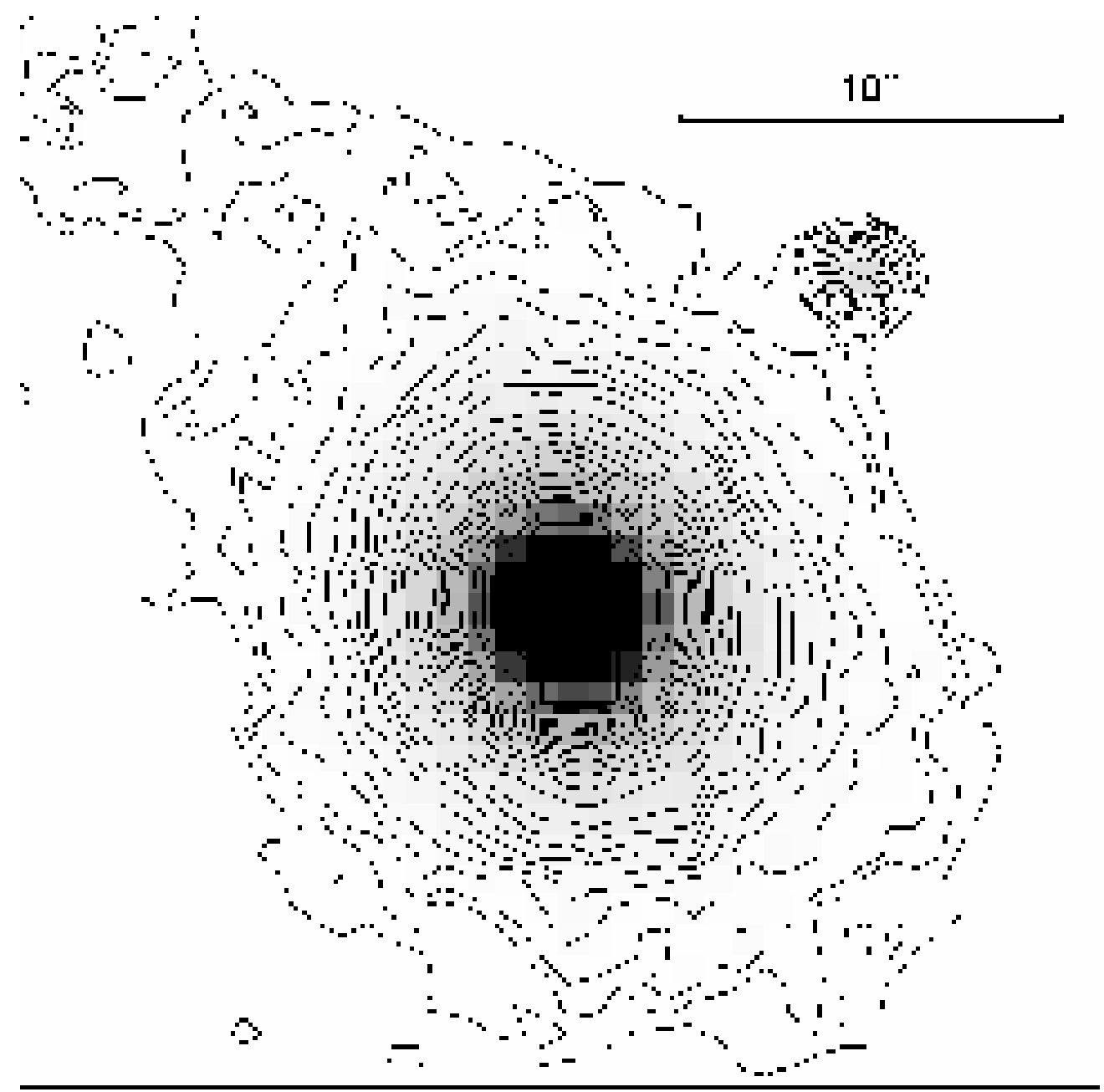

Fig. 29.- Same as Figure 23 for HCG 94b. 

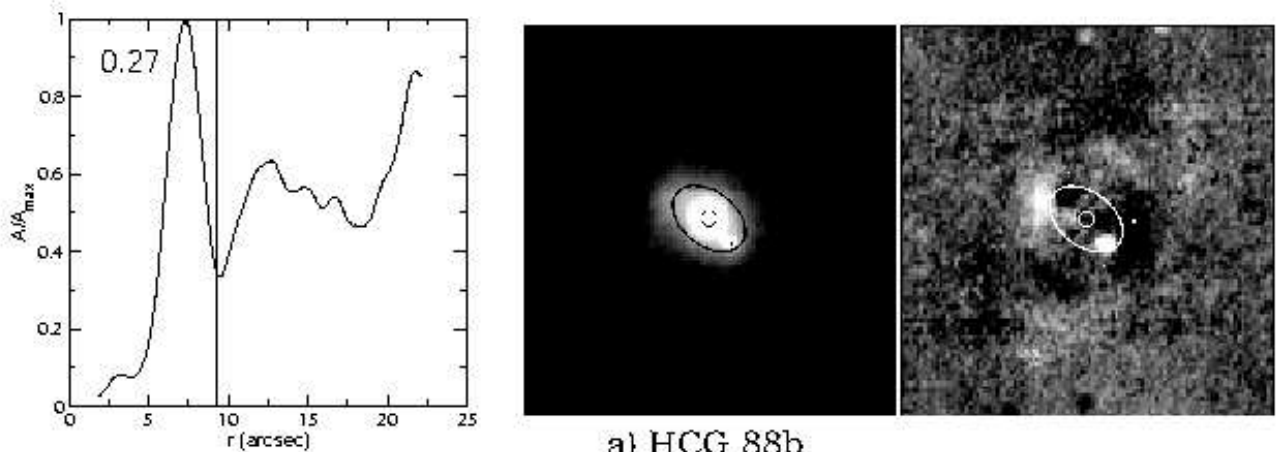

a) HCG $88 \mathrm{~b}$
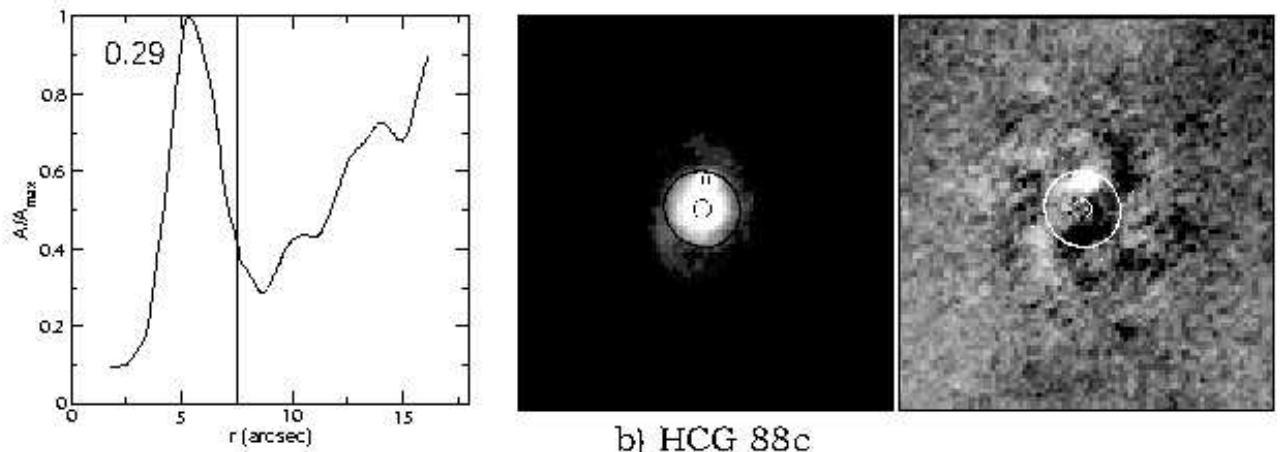

b) HCG $88 \mathrm{c}$
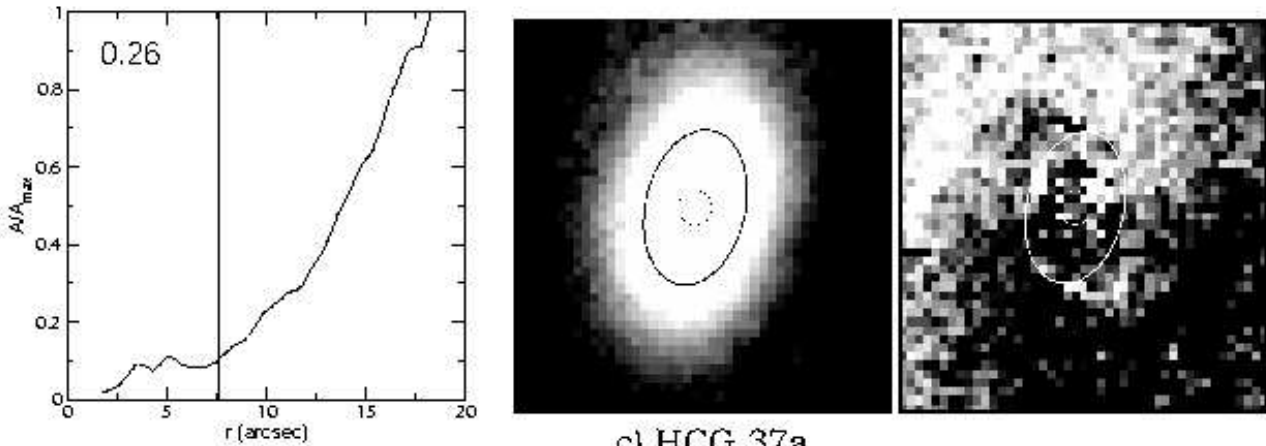

c) HCG $37 \mathrm{a}$
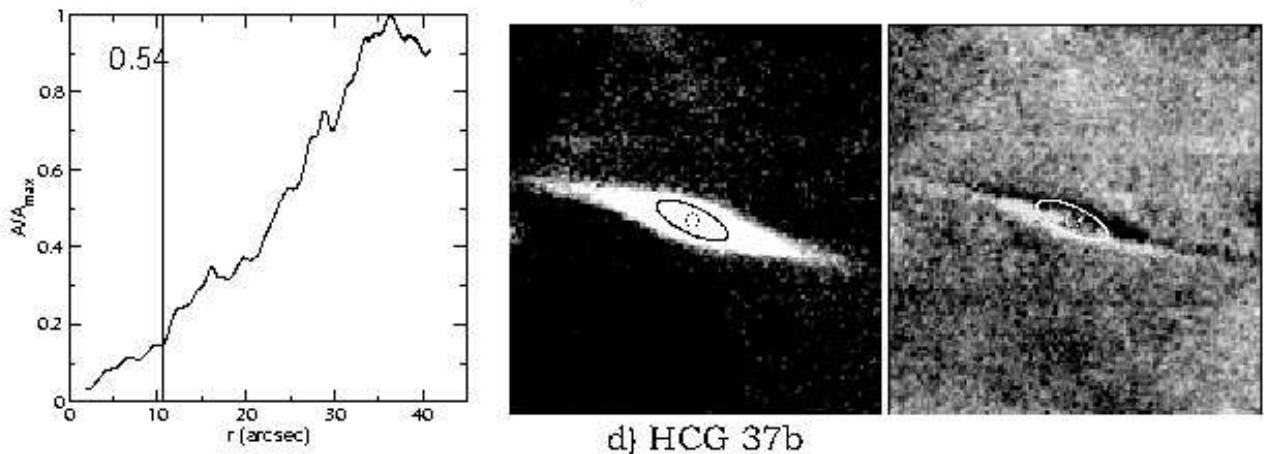

d) HCG $37 \mathrm{~b}$

Fig. 30.- Results of the asymmetry analysis. The image in the center is the original image, cleaned (as much as possible) of all foreground objects. The inner circle is the size of the PSF and the ellipse is at isophote $r_{50}$. The image to the right is the residual image obtained by subtracting the rotated image from the original one. The two images are shown at the same scale (north up and east to the left). The graphics to the left give the relative asymmetry intensity $\left(A / A_{\max }\right)$ at different radius. The maximum value is indicated in the upper left corner. The vertical line is draw at $r_{50}$. 

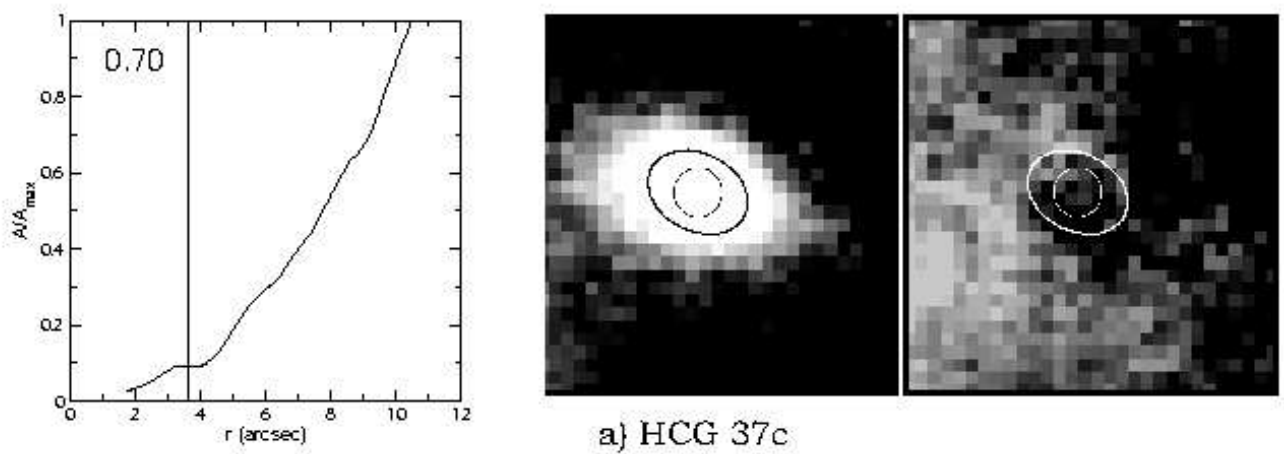

a) $\mathrm{HCG} 37 \mathrm{c}$
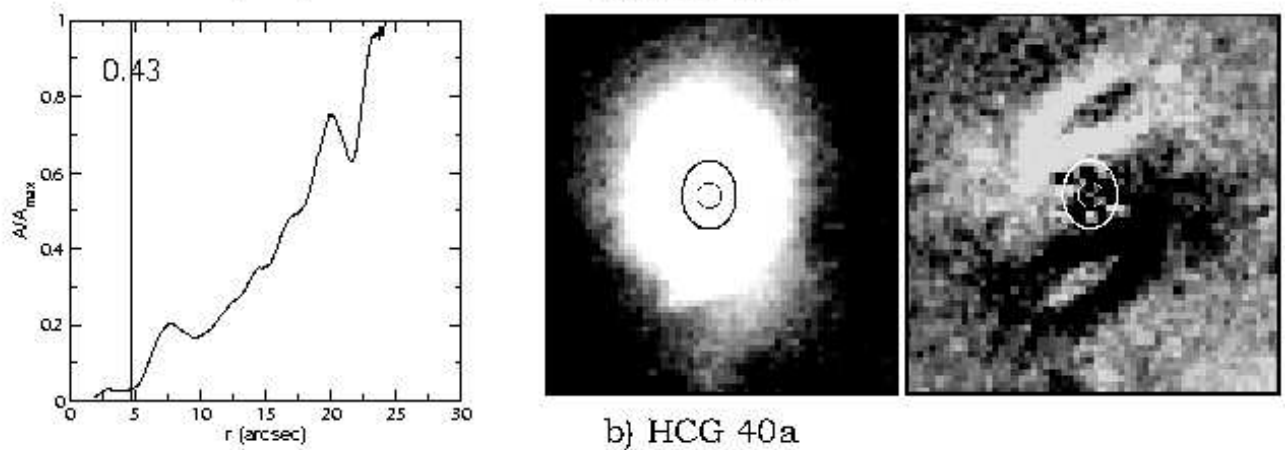

b) $\mathrm{HCG} 40 \mathrm{a}$
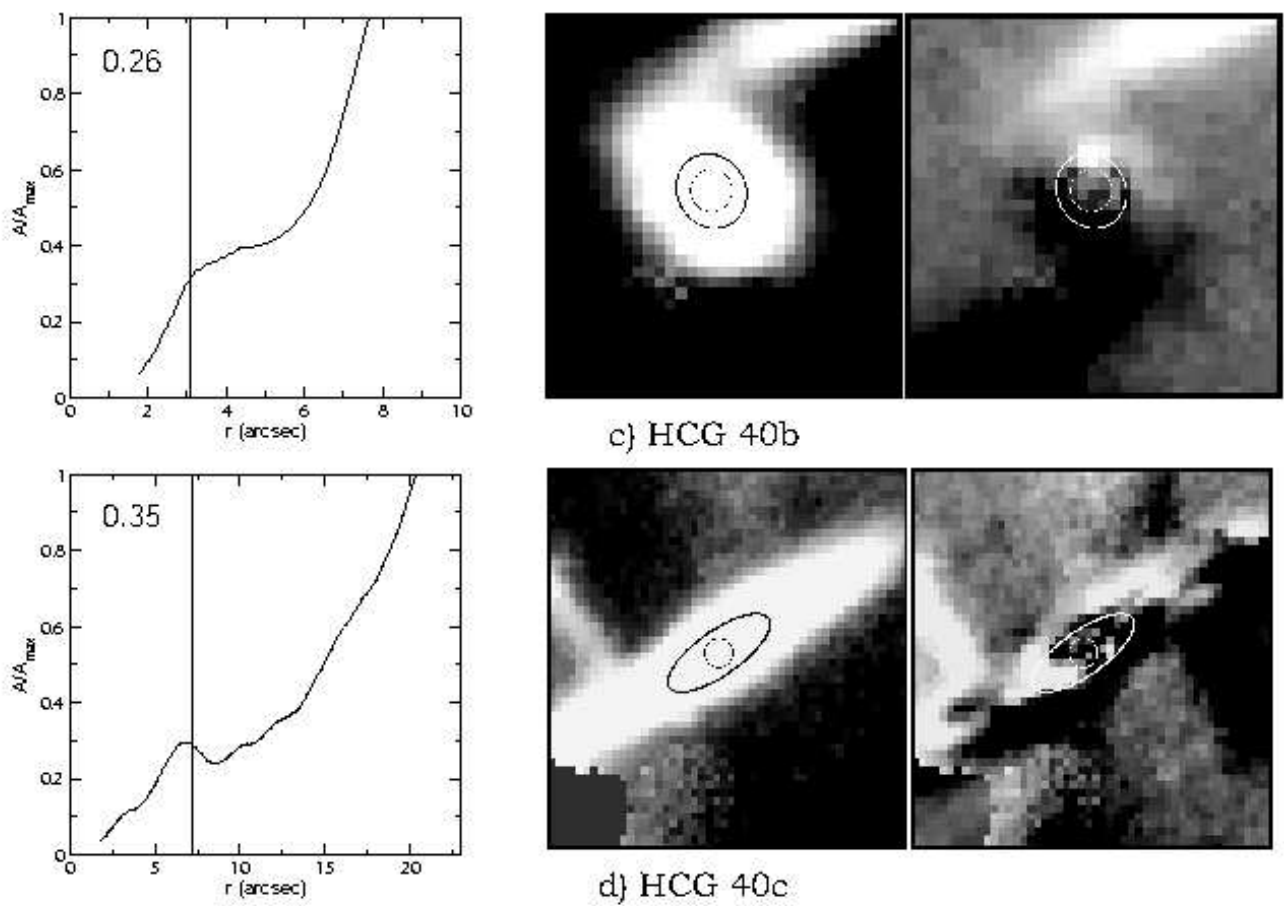

c) HCG $40 \mathrm{~b}$

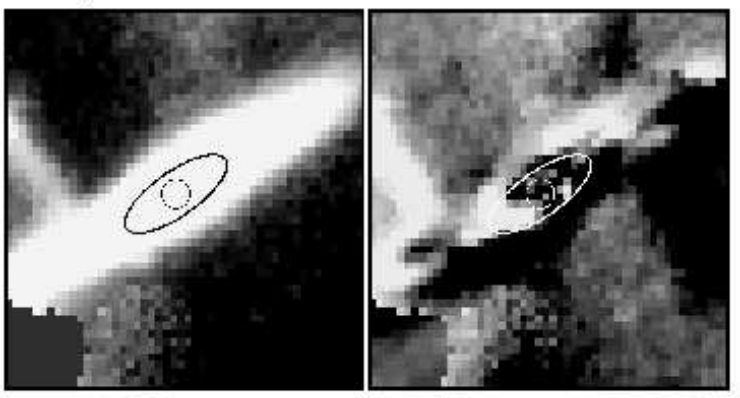

d) $\mathrm{HCG} 40 \mathrm{c}$

Fig. 31. - Same as Figure 30. 

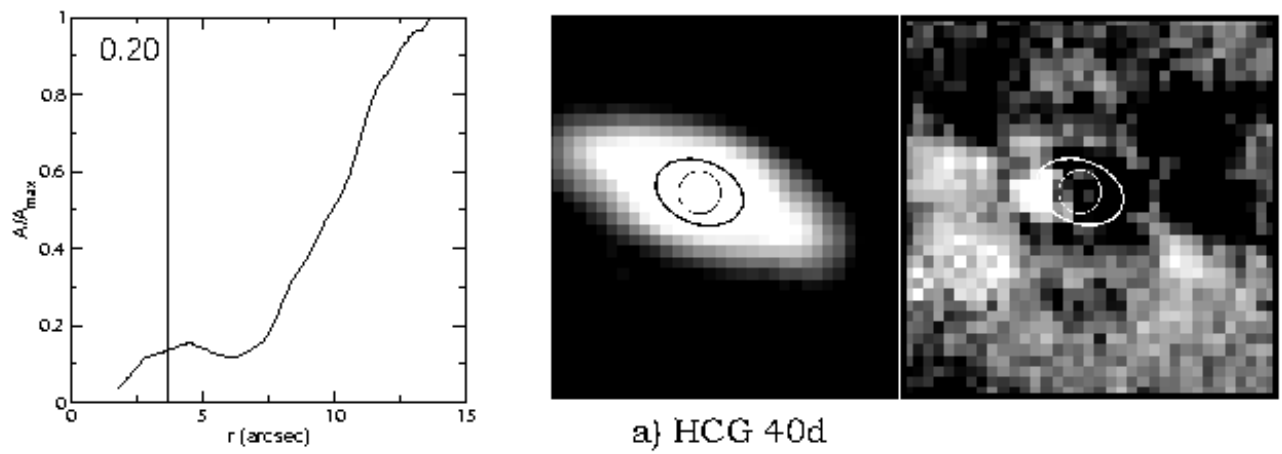

a) $\mathrm{HCG} 40 \mathrm{~d}$
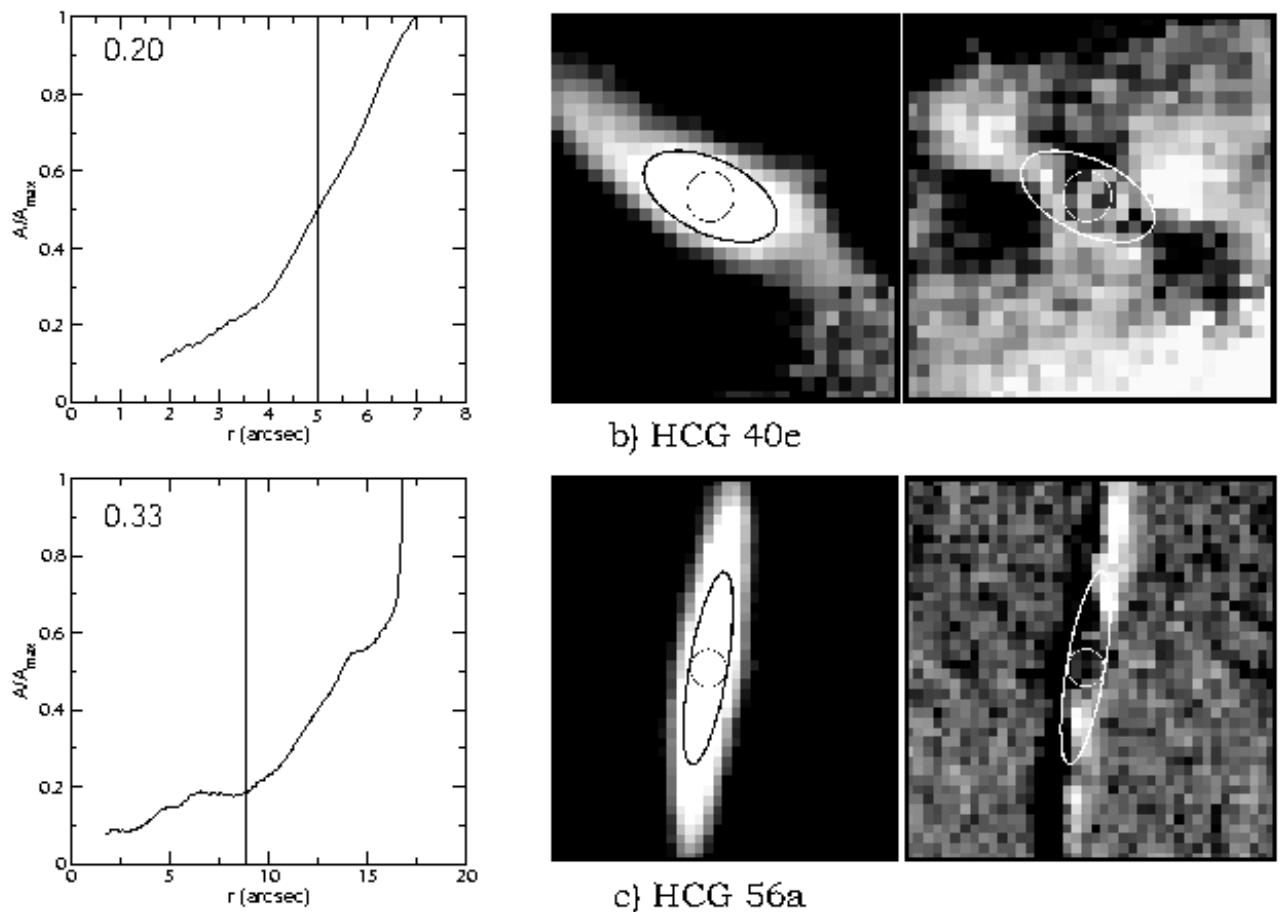

c) $\mathrm{HCG} 56 \mathrm{a}$
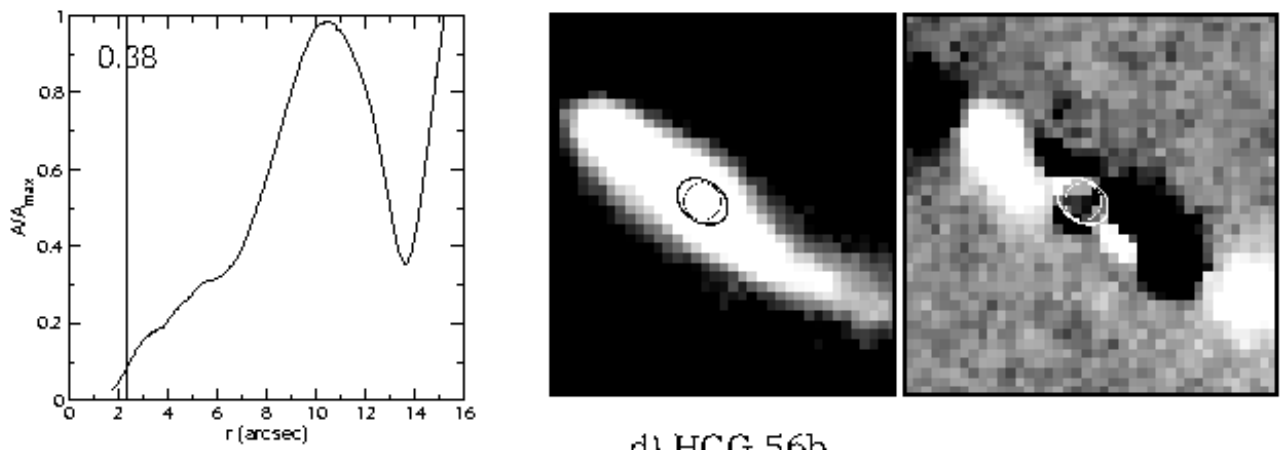

d) HCG 56b

Fig. 32.- Same as Figure 30. 

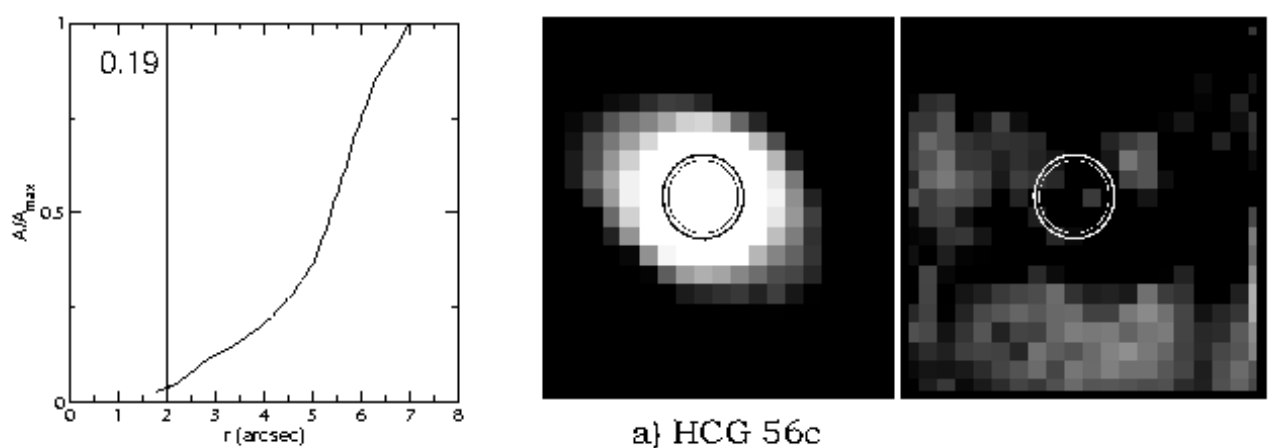

a) $\mathrm{HCG} 56 \mathrm{c}$
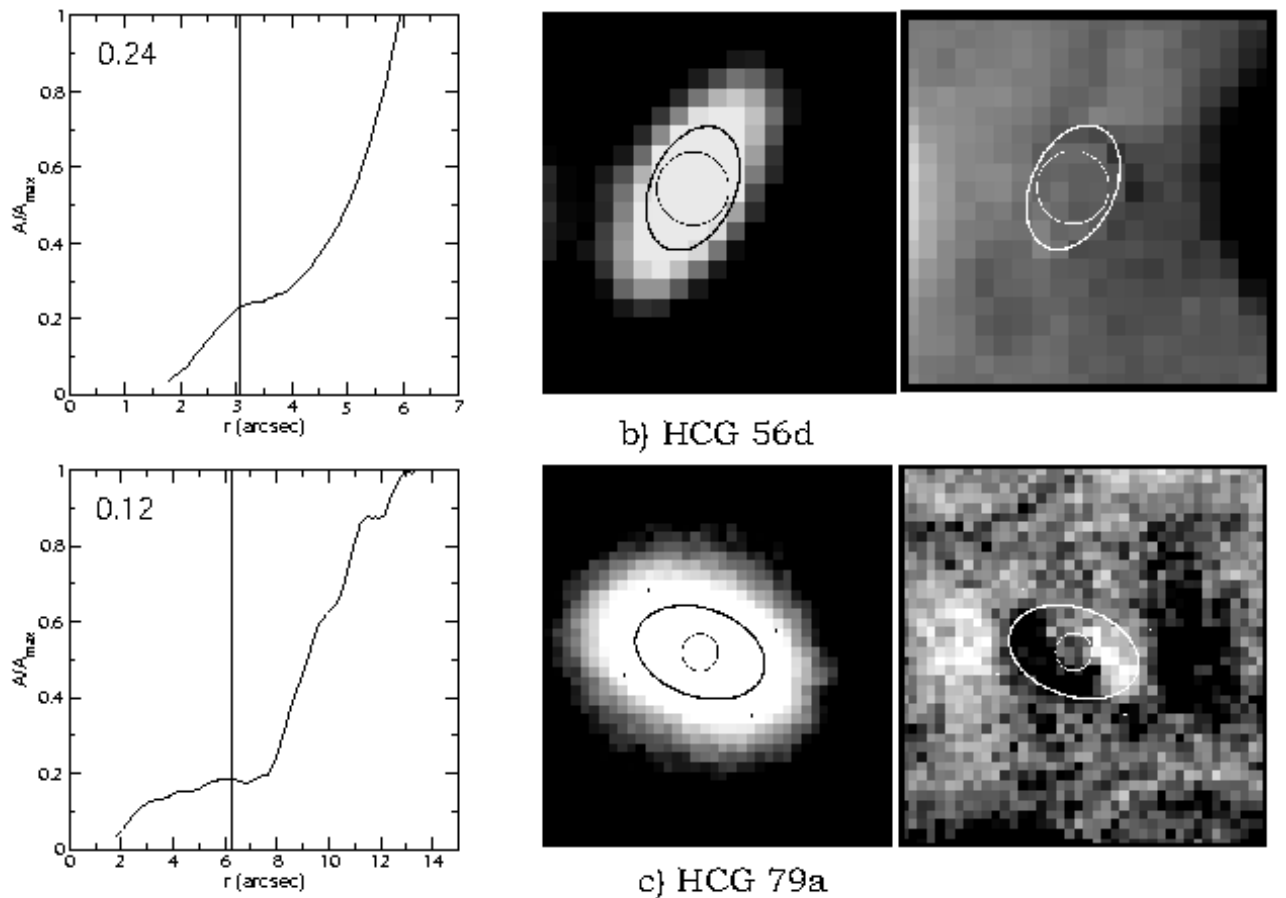

b) HCG $56 \mathrm{~d}$

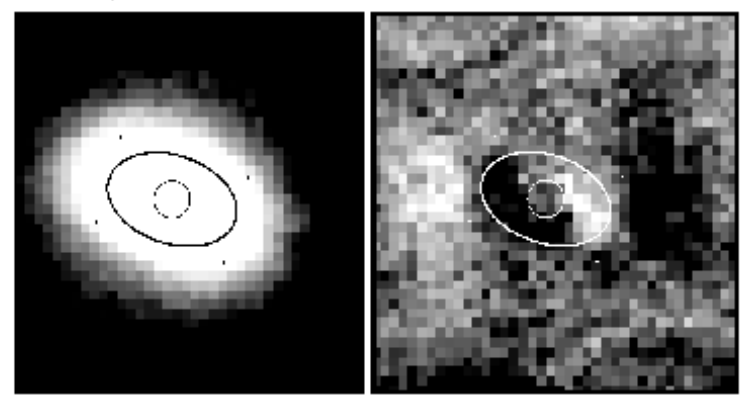

c) $\mathrm{HCG} 79 \mathrm{a}$
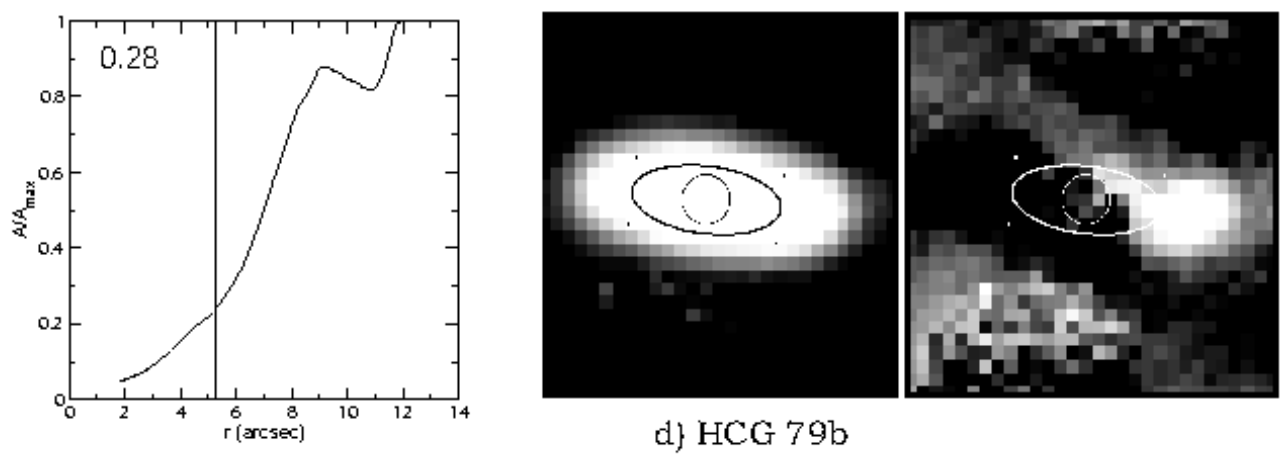

d) HCG $79 b$

Fig. 33. - Same as Figure 30. 

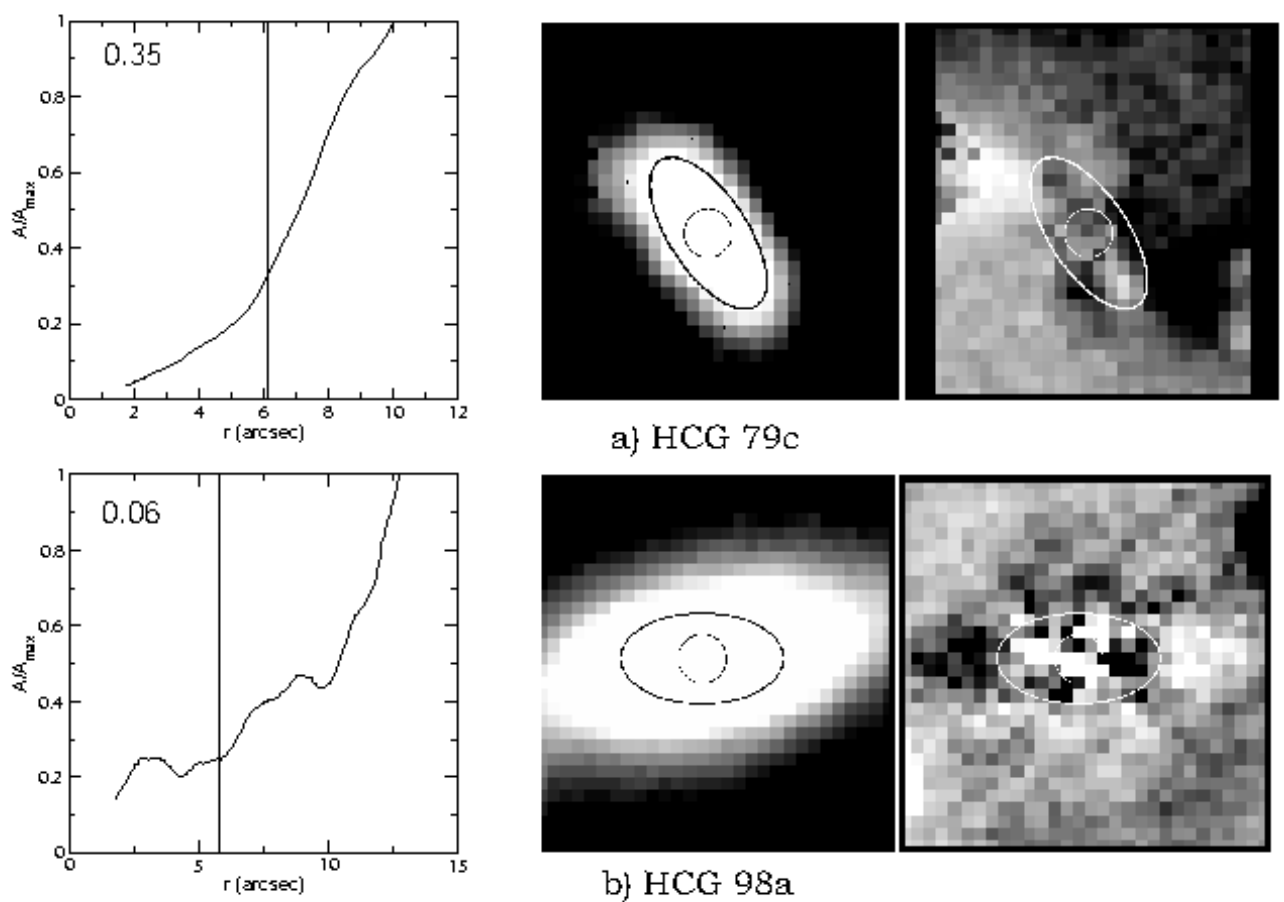

a) $\mathrm{HCG} 79 \mathrm{c}$

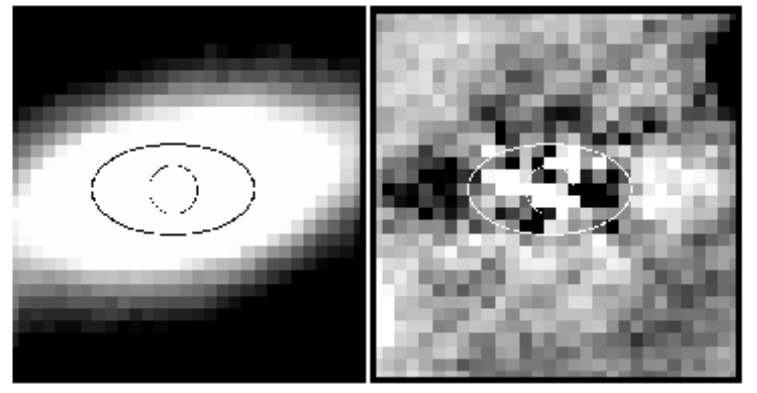

b) HCG $98 \mathrm{a}$
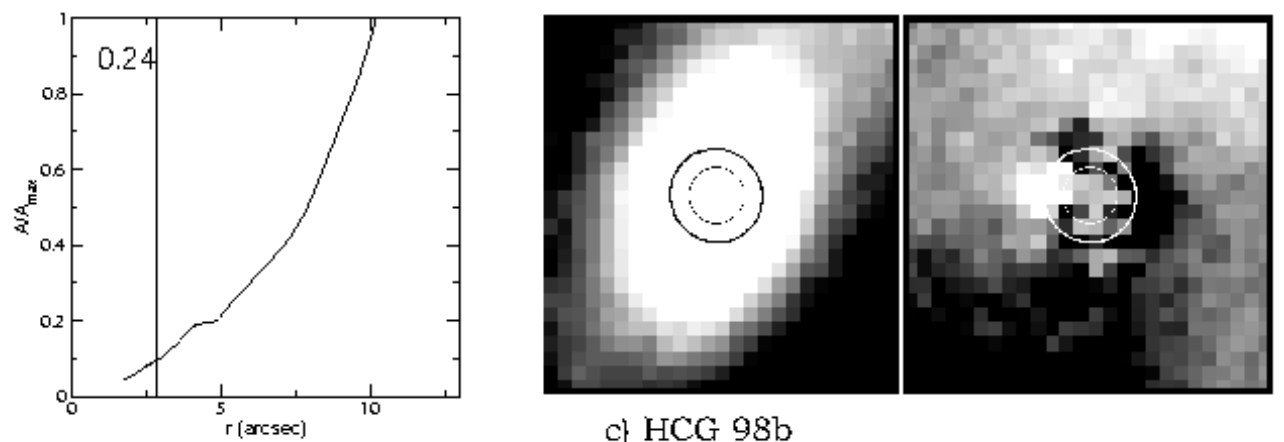

c) HCG $98 \mathrm{~b}$
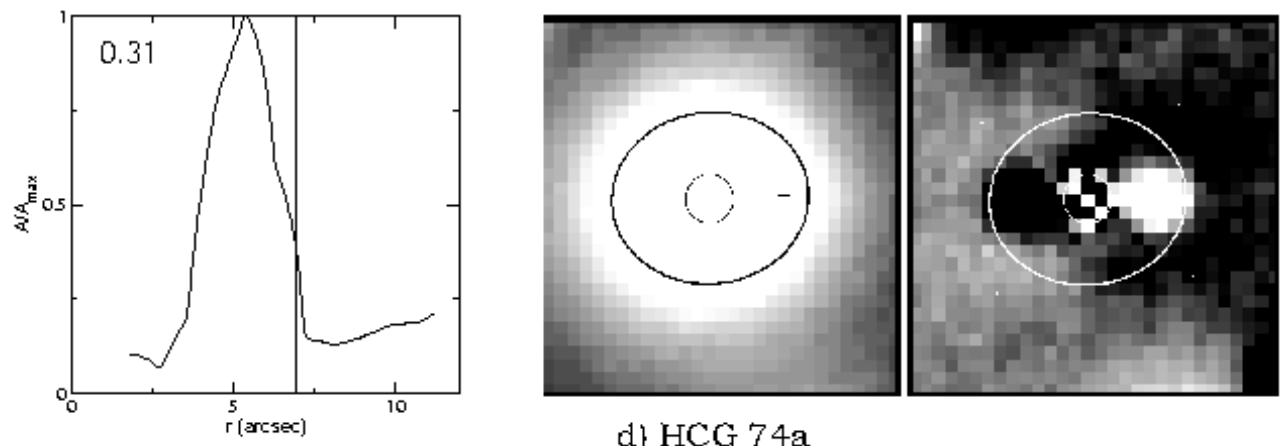

d) $\mathrm{HCG} 74 \mathrm{a}$

Fig. 34. - Same as Figure 30. 

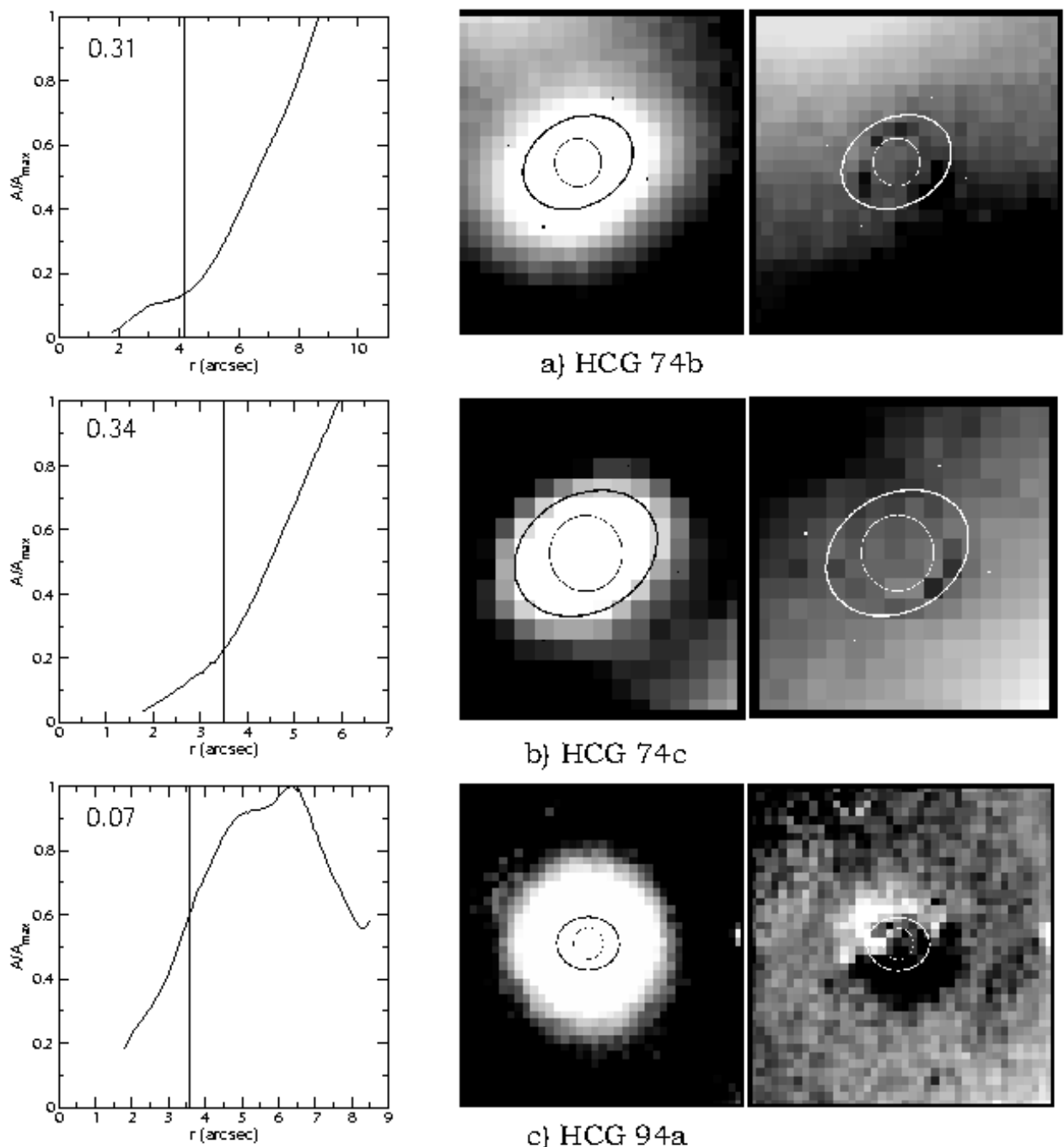

b) HCG $74 \mathrm{c}$
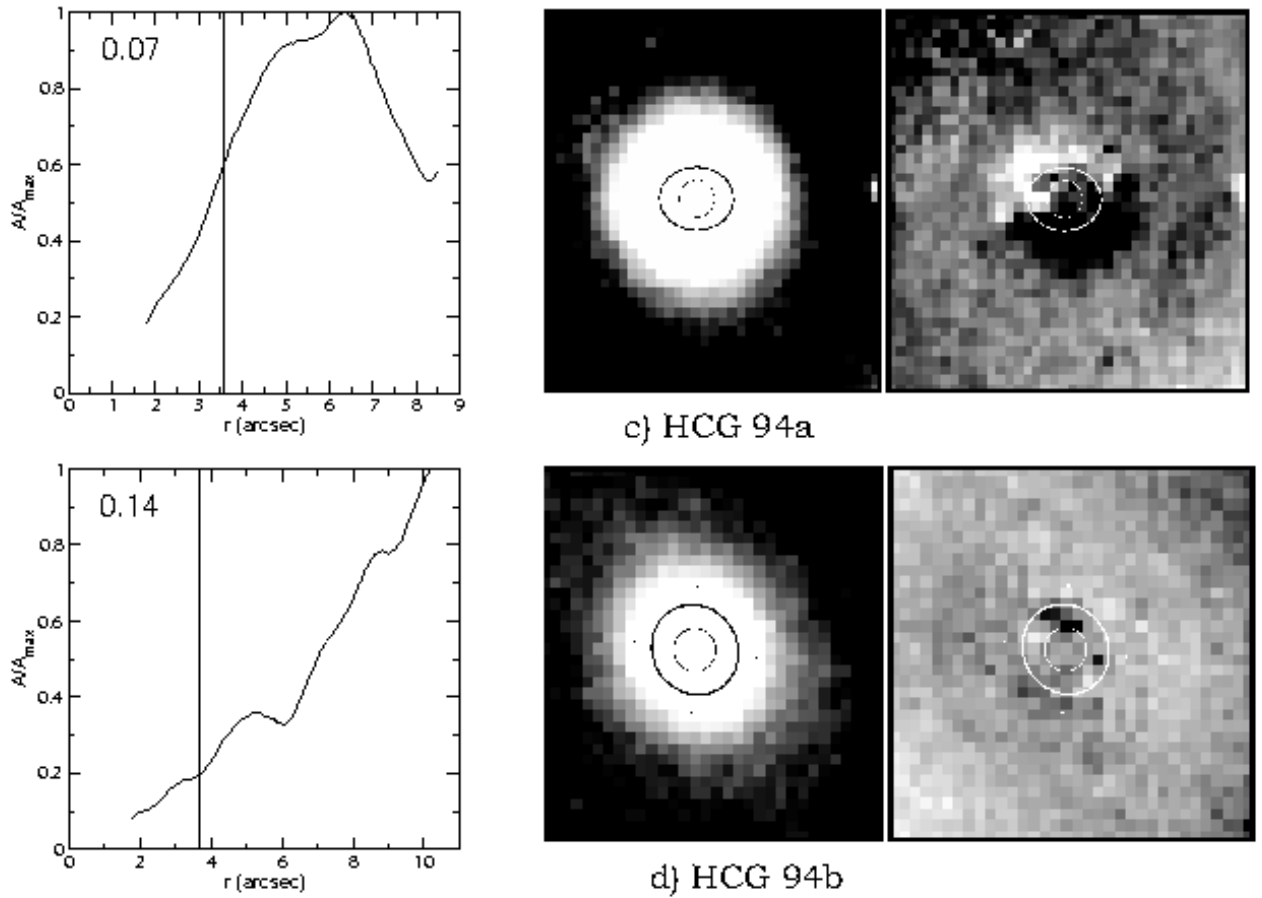

c) $\mathrm{HCG} 94 \mathrm{a}$

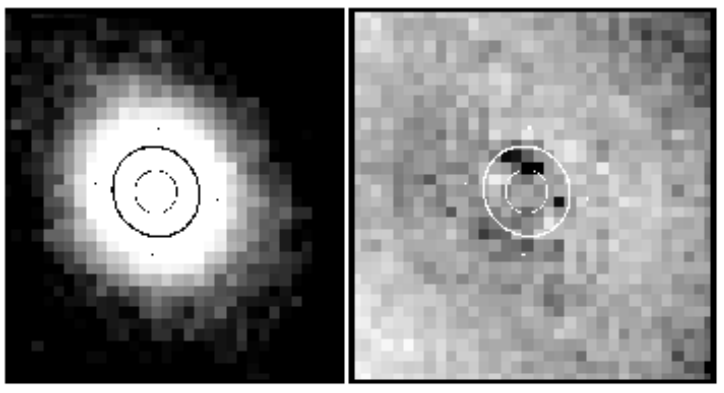

d) HCG 94b

Fig. 35.- Same as Figure 30. 
Table 1. Properties of the observed groups

\begin{tabular}{ccccccc}
\hline \hline Group & $\begin{array}{c}\text { R.A. } \\
(J 2000) \\
(1)\end{array}$ & $\begin{array}{c}\text { Dec. } \\
(\mathrm{J} 2000) \\
(3)\end{array}$ & $\begin{array}{c}\sigma \\
\left(\mathrm{km} \mathrm{s} \mathrm{s}^{-1}\right) \\
(4)\end{array}$ & $\begin{array}{c}v_{o} \\
\left(\mathrm{~km} \mathrm{~s}^{-1}\right) \\
(5)\end{array}$ & $\begin{array}{c}\text { Angular size } \\
(\text { arcmin }) \\
(6)\end{array}$ & $\begin{array}{c}\text { Evolutionary } \\
\text { type } \\
(7)\end{array}$ \\
\hline HCG 37 & 091335.6 & +300051 & 692 & 6685 & 3.2 & $\mathrm{~B}$ \\
HCG 40 & 093854.5 & -045107 & 251 & 6685 & 1.7 & $\mathrm{~B}$ \\
HCG 56 & 113239.6 & +525625 & 282 & 8094 & 2.1 & $\mathrm{~B}$ \\
HCG 74 & 151928.2 & +205337 & 537 & 11962 & 1.9 & $\mathrm{C}$ \\
HCG 79 & 155911.9 & +204531 & 229 & 4347 & 1.9 & $\mathrm{~A}$ \\
HCG 88 & 205222.8 & -054528 & 27 & 6026 & 5.2 & $\mathrm{C}$ \\
HCG 94 & 231716.5 & +184311 & 832 & 12501 & 2.8 & $\mathrm{~B}$ \\
HCG 98 & 235412.7 & +002224 & 204 & 7974 & 2.4 & \\
\hline
\end{tabular}

Note. - Columns are: (1) Hickson's group identifications (Hickson 1982); (2) Right ascension (NASA/IPAC Extragalactic Database (NED)); (3) Declination (NED); (4) Velocity dispersion of the group (Hickson 1992); (5) Heliocentric group velocity (NED) ; (6) Angular size subtended in the sky (NED); (7) Evolutionary type (Coziol et al. 2004). 
Table 2. Properties of the observed galaxies

\begin{tabular}{|c|c|c|c|c|c|c|}
\hline $\begin{array}{r}\mathrm{HCG} \\
(1)\end{array}$ & $\begin{array}{c}\text { Radial vel. } \\
\left(k m s^{-1}\right) \\
(2)\end{array}$ & $\begin{array}{l}\text { Morphology } \\
\text { (3) }\end{array}$ & $\begin{array}{l}E W_{a c t} \\
(\AA) \\
(4)\end{array}$ & $\begin{array}{c}\text { Nuclear activity } \\
\text { (5) }\end{array}$ & $\begin{array}{l}t_{J} \\
(s) \\
(6)\end{array}$ & $\begin{array}{r}t_{K^{\prime}} \\
(s) \\
(7)\end{array}$ \\
\hline $88 \mathrm{~b}$ & 6010 & $\mathrm{SBb}$ & -6 & dSy2 & 3600 & 450 \\
\hline $88 c$ & 6083 & $\mathrm{Sc}$ & -30 & SFG & 3600 & 200 \\
\hline $37 \mathrm{a}$ & 6745 & E7 & -3 & dLINER & 2250 & 860 \\
\hline $37 \mathrm{~b}$ & 6741 & $\mathrm{Sbc}$ & -1 & LINER & 2250 & 860 \\
\hline $37 \mathrm{c}$ & 7357 & S0a & 0 & LLAGN? & 2250 & 860 \\
\hline $40 \mathrm{a}$ & 6628 & E3 & 1 & no emission & 1600 & 900 \\
\hline $40 \mathrm{~b}$ & 6842 & So & 3 & no emission & 1600 & 900 \\
\hline $40 \mathrm{c}$ & 6890 & $\mathrm{Sbc}$ & -12 & SFG & 1600 & 900 \\
\hline $40 \mathrm{~d}$ & 6492 & $\mathrm{SBa}$ & -40 & SFG & 1600 & 900 \\
\hline $40 \mathrm{e}$ & 6625 & $\mathrm{Sc}$ & -3 & SFG? & 1600 & 900 \\
\hline $56 \mathrm{a}$ & 8245 & $\mathrm{Sc}$ & -17 & SFG & 3600 & 900 \\
\hline $56 \mathrm{~b}$ & 7919 & SB0 & -52 & Sy2 & 3600 & 900 \\
\hline $56 \mathrm{c}$ & 8110 & S0 & 1 & no emission & 3600 & 900 \\
\hline $56 \mathrm{~d}$ & 8346 & So & -24 & SFG & 3600 & 900 \\
\hline $56 \mathrm{e}$ & 7924 & So & -14 & SFG? & 3600 & 900 \\
\hline $79 \mathrm{a}$ & 4292 & $\mathrm{Sa}$ & -5 & LINER & 3600 & $\ldots$ \\
\hline $79 b$ & 4446 & So & -6 & SFG? & 3600 & $\ldots$ \\
\hline $79 \mathrm{c}$ & 4146 & So & 5 & no emission & 3600 & $\ldots$ \\
\hline $98 \mathrm{a}$ & 7855 & SB0 & 9 & no emission & 3600 & 1440 \\
\hline $98 \mathrm{~b}$ & 7959 & So & 3 & no emission & 3600 & 1440 \\
\hline $74 \mathrm{a}$ & 12255 & E1 & 1 & no emission & 4400 & 1160 \\
\hline $74 \mathrm{~b}$ & 12110 & E3 & 2 & no emission & 4400 & 1160 \\
\hline $74 \mathrm{c}$ & 12266 & So & 3 & no emission & 4400 & 1160 \\
\hline $94 \mathrm{a}$ & 12040 & E1 & 5 & no emission & 1300 & 650 \\
\hline $94 \mathrm{~b}$ & 11974 & E3 & 4 & no emission & 1300 & 650 \\
\hline
\end{tabular}

Note. - Columns are: (1) Galaxy identification (Hickson 1982); (2) Radial velocity (NED) (3) Morphological type (Hickson 1989), except for HCG 79a, which was taken from NED; (4, 5) Spectral index and activity type (Coziol et al. 2004); (6) Total exposure time in J; (7) Total exposure time in K'; 
Table 3. Near Infrared magnitudes from 2MASS

\begin{tabular}{ccccc}
\hline \hline HCG & $m_{J} \pm \Delta m_{J}$ & $m_{H} \pm \Delta m_{H}$ & $m_{K} \pm \Delta m_{K}$ & $B-R$ \\
& & & & \\
$(1)$ & $(2)$ & $(3)$ & $(4)$ & $(5)$ \\
\hline 88b & $11.04 \pm 0.04$ & $10.38 \pm 0.04$ & $10.02 \pm 0.05$ & 1.51 \\
$88 \mathrm{c}$ & $12.44 \pm 0.04$ & $11.77 \pm 0.05$ & $11.89 \pm 0.09$ & 0.99 \\
$37 \mathrm{a}$ & $10.25 \pm 0.02$ & $9.60 \pm 0.02$ & $9.32 \pm 0.03$ & 1.79 \\
$37 \mathrm{~b}$ & $11.60 \pm 0.03$ & $10.88 \pm 0.03$ & $10.56 \pm 0.04$ & 1.99 \\
$37 \mathrm{c}$ & $\ldots$ & $\ldots$ & $\ldots$ & 1.76 \\
$40 \mathrm{a}$ & $10.40 \pm 0.02$ & $9.71 \pm 0.02$ & $9.46 \pm 0.03$ & 1.75 \\
$40 \mathrm{~b}$ & $11.47 \pm 0.03$ & $10.76 \pm 0.03$ & $10.52 \pm 0.04$ & 1.84 \\
$40 \mathrm{c}$ & $11.12 \pm 0.03$ & $10.38 \pm 0.03$ & $10.12 \pm 0.05$ & 2.00 \\
$40 \mathrm{~d}$ & $11.78 \pm 0.03$ & $11.02 \pm 0.03$ & $10.67 \pm 0.04$ & 1.56 \\
$40 \mathrm{e}$ & $13.23 \pm 0.08$ & $12.50 \pm 0.08$ & $12.18 \pm 0.08$ & 1.84 \\
$56 \mathrm{a}$ & $12.48 \pm 0.07$ & $11.37 \pm 0.08$ & $10.48 \pm 0.05$ & 1.51 \\
$56 \mathrm{~b}$ & $11.65 \pm 0.07$ & $10.82 \pm 0.12$ & $10.01 \pm 0.06$ & 1.43 \\
$56 \mathrm{c}$ & $\ldots$ & $\ldots$ & $\ldots$ & 1.52 \\
$56 \mathrm{~d}$ & $\ldots$ & $\ldots$ & $\ldots$ & 1.62 \\
$56 \mathrm{e}$ & $14.01 \pm 0.05$ & $13.35 \pm 0.09$ & $12.97 \pm 0.08$ & 1.20 \\
$79 \mathrm{a}$ & $11.50 \pm 0.02$ & $10.80 \pm 0.03$ & $10.53 \pm 0.04$ & 1.60 \\
$79 \mathrm{~b}$ & $11.24 \pm 0.03$ & $10.47 \pm 0.03$ & $10.59 \pm 0.06$ & 1.44 \\
$79 \mathrm{c}$ & $\ldots$ & $\ldots$ & $\cdots$ & 1.27 \\
$98 \mathrm{a}$ & $10.40 \pm 0.07$ & $9.67 \pm 0.09$ & $9.73 \pm 0.03$ & 1.60 \\
$98 \mathrm{~b}$ & $\ldots$ & $\ldots$ & $\cdots$ & 1.58 \\
$74 \mathrm{a}$ & $10.91 \pm 0.03$ & $10.06 \pm 0.02$ & $9.85 \pm 0.04$ & 1.90 \\
$74 \mathrm{~b}$ & $\ldots$ & $\ldots$ & $\cdots$ & 1.88 \\
$74 \mathrm{c}$ & $\ldots$ & $\ldots$ & $\cdots$ & 1.83 \\
$94 \mathrm{a}$ & $10.53 \pm 0.02$ & $9.65 \pm 0.03$ & $9.60 \pm 0.04$ & 1.67 \\
$94 \mathrm{~b}$ & $\ldots$ & $\ldots$ & $\cdots$ & 1.63 \\
\hline & & & &
\end{tabular}

Note. - Columns are: (1) HCG identification; (2) J magnitude; (3) H magnitude; (4) K magnitude; (5) B-R color (Hickson et al. 1989 
Table 4. Asymmetry analysis summary

\begin{tabular}{|c|c|c|c|}
\hline $\begin{array}{c}\text { Evolutionary } \\
\text { type } \\
(1)\end{array}$ & $\begin{array}{l}\mathrm{HCG} \\
(2)\end{array}$ & $\begin{array}{c}r_{50} \\
(\operatorname{arcsec}) \\
(3)\end{array}$ & asymmetric properties \\
\hline \multirow[t]{2}{*}{ A } & $88 \mathrm{~b}$ & 9.3 & Tidal pair with merger - blue nucleus \\
\hline & $+88 \mathrm{c}$ & 7.5 & Spiral arms - blue nucleus \\
\hline \multirow[t]{18}{*}{ B } & $37 \mathrm{a}$ & 7.7 & Light excess toward $b$ \\
\hline & $37 \mathrm{~b}$ & 10.6 & Light excess toward a \\
\hline & $37 \mathrm{c}$ & 3.7 & Symmetric - blue nucleus \\
\hline & $40 \mathrm{a}$ & 4.7 & Tidal pair with bridge toward c - blue nucleus? \\
\hline & $40 \mathrm{~b}$ & 3.1 & Light excess toward c - merger? - blue nucleus? \\
\hline & $40 \mathrm{c}$ & 7.1 & Tidal pair - bridges with e, $\mathrm{b}$ and a \\
\hline & $40 \mathrm{~d}$ & 3.7 & Tidal pair with merger? - light excesses toward a? \\
\hline & $40 \mathrm{e}$ & 5.0 & Tidal pair with bridge toward $\mathrm{c}-$ blue nucleus \\
\hline & $-56 \mathrm{a}$ & 8.8 & Light excess toward other members \\
\hline & $-56 b$ & 2.4 & Tidal pair with bridge toward c \\
\hline & $56 \mathrm{c}$ & 2.0 & Symmetric. \\
\hline & $56 \mathrm{~d}$ & 3.1 & Symmetric. \\
\hline & $56 \mathrm{e}$ & 2.6 & $\cdots$ \\
\hline & $79 \mathrm{a}$ & 6.3 & Tidal pair with nucleus \\
\hline & $+79 \mathrm{~b}$ & 5.3 & Bridge toward c - tidal pair with tail \\
\hline & $79 \mathrm{c}$ & 6.1 & Symmetric? - bridge toward b \\
\hline & $+98 \mathrm{a}$ & 5.8 & Tidal pair with nucleus - merger? - blue nucleus? \\
\hline & $98 \mathrm{~b}$ & 2.9 & Light excess toward 98a - merger? \\
\hline \multirow[t]{5}{*}{$\mathrm{C}$} & $74 \mathrm{a}$ & 7.0 & Tidal pair with merger \\
\hline & $74 \mathrm{~b}$ & 4.1 & Symmetric \\
\hline & $74 \mathrm{c}$ & 3.5 & Symmetric \\
\hline & $+94 \mathrm{a}$ & 3.6 & Light excess - merger? - blue nucleus \\
\hline & $94 \mathrm{~b}$ & 3.7 & Light excess - blue nucleus \\
\hline
\end{tabular}

Note. - Columns are: (1) Global activity type; (2) HCG identification (a + sign identifies galaxies with blue 2MASS colors and a - sign identifies galaxies affected by AGN or star formation with dust extinction); (3) Radius containing $50 \%$ of total light; (4) Observed asymmetric properties.

Table 5. Asymmetry vs. activity analysis

\begin{tabular}{lccc}
\hline \hline & \multicolumn{3}{c}{ Evolutionary type } \\
& A & B & C \\
\hline Nb. of gal. & 2 & 18 & 5 \\
Nb. active & 2 & 12 & 0 \\
Nb. late-type & 2 & 5 & 0 \\
Nb. asymmetric & 1 & 13 & 3 \\
\hline
\end{tabular}

